\title{
DISEÑO DE MODELOS Y ALGORITMOS PARA EL PROCESAMIENTO DE DATOS SAR POLARIMÉTRICOS
}

\author{
Tesis de Doctorado
}

\section{Juan Ignacio FERNÁNDEZ MICHELLI}

Presentada ante la Facultad de Ingeniería de la Universidad Nacional de La Plata como requisito para la obtención del grado académico de

\section{DOCTOR EN INGENIERÍA}

\author{
Dirección de tesis: \\ Director: Dr. Martín Hurtado \\ Codirector: Dr. Javier A. Areta \\ Jurado de tesis: \\ Dr. Alejandro César Frery Orgambide \\ Dra. María Magdalena Lucini \\ Dr. Pablo Andrés Euillades
}

Fecha de defensa oral y pública: 30 de marzo de 2016 


\section{Resumen}

Un sistema SAR es un radar aerotransportado o satelital que sensa la superficie terrestre y forma imágenes para su estudio. Funciona emitiendo ondas electromagnéticas sobre la zona de interés y recolectando las reflexiones producidas. Las ondas reflejadas contienen información acerca del comportamiento del terreno ante la interacción con dichas ondas, a partir de la cual es posible inferir características físicas del terreno subyacente.

El proceso de interpretación de las imágenes SAR está basado principalmente en la combinación de dos disciplinas que se complementan: el electromagnetismo y procesamiento estadístico de señales. La extracción de la información de una imagen SAR está fuertemente ligada al modelo utilizado para describir los datos, que a su vez encuentra su fundamento en el comportamiento electromagnético de las ondas reflejadas.

Actualmente, una de las aplicaciones más importantes del procesamiento de datos SAR polarimétricos es la clasificación y segmentación de datos. Muchos algoritmos, tanto supervisados como no supervisados, han sido desarrollados para llevar a cabo esta tarea. En los primeros, se seleccionan datos de entrenamiento basados en mapas del terreno, que contienen las características que se desean identificar. Por otro lado, en los algoritmos no supervisados, no se utilizan datos previos. El algoritmo clasifica la imagen de forma automática organizando los píxeles en diferentes clases bajo cierto criterio. En ambos casos, las características a identificar están embebidas en una matriz compleja de nueve elementos que describe a los datos polarimétricos. Los criterios de formación de clases están basados en el modelo estadístico asignado a 
estas matrices (o a combinaciones de sus elementos) y en distancias estadísticas que de ellos se derivan.

Este trabajo realiza su principal aporte en el campo de la clasificación de datos polarimétricos. Para ello, se estudia en primer lugar la geometría del problema SAR y el proceso de formación de imágenes partiendo de los datos crudos. Esto permite entender cabalmente qué magnitudes son las utilizadas para componer las imágenes e interpretarlas correctamente. Luego se aborda el estudio de los modelos estadísticos utilizados en SAR, la distribución Wishart que describe datos polarimétricos y el modelo multiplicativo que da cuenta del alejamiento del modelo gaussiano. En base a esto, se propone la utilización de un modelo de mezclas de densidades para los datos y se desarrollan algoritmos de clasificación propios basados en ellos. Para el caso de datos homogéneos donde el modelo gaussiano es válido, se utiliza un modelo de mezcla de densidades gaussianas multivariante. Para el caso de datos heterogéneos, se utiliza la mezcla de densidades $\mathcal{G}_{p}^{0}$ que tiene en cuenta la textura. En ambos casos, los algoritmos de clasificación desarrollados se basan el método ExpectationMaximization y clasificación MAP. Son algoritmos no supervisados con una instancia de selección de modelo que permite detectar automáticamente la cantidad de clases relevantes en la escena. Esto asegura el modelo de menor complejidad para la escena dada en función de la distribución utilizada, y por otro lado, evita la necesidad de especificar a priori el número de clases. 


\section{Agradecimientos}

En primer lugar, quiero agradecer a mis padres María Inés y Jaime, y a mi hermana, María Virginia, por su apoyo tanto en esta etapa como en todas las que he decidido atravesar en la vida.

Al Instituto de Investigaciones en Electrónica, Control y Procesamiento de Señales - LEICI - por proporcionar el lugar y material de trabajo.

A las instituciones que financiaron el desarrollo de la tesis: el Consejo Nacional de Investigaciones Científicas y Técnicas y a la Facultad de Ingeniería de la Universidad Nacional de La Plata.

A mis directores, Dr. Martín Hurtado y Dr. Javier Areta, a quienes agradezco haber aceptado dirigirme en esta tesis brindándome su constante apoyo, predisposición y conocimientos.

A mis compañeros, Juan Pablo, Mariano y Sebastián, por su inestimable aporte de ideas y experiencias, y por brindarme su amistad.

Al Dr. Carlos Muravchik y a todo el grupo de procesamiento de señales que él encabeza, por permitirme integrar un grupo en el que la calidad académica, el respeto y la camaradería proporcionan un ámbito inmejorable para el desarrollo del doctorado.

Finalmente a Paula por su incondicional apoyo, y por entender mis ausencias y vigilias. 


\section{Índice general}

Îndice general vii

Lista de abreviaturas $\quad$ xi

1. Introducción 1

1.1. Resultados preexistentes ................... . 3

1.2. Contribuciones originales . . . . . . . . . . . . . . 4

1.3. Organización de la tesis . . . . . . . . . . . . 7

2. Radar de Apertura Sintética $\quad 9$

2.1. Geometría del SAR aerotransportado . . . . . . . . . . . . 10

2.2. Arreglo de datos . . . . . . . . . . . . . . . . . . 11

2.3. Señales SAR . . . . . . . . . . . . . . . . . . . . 13

2.3.1. Señal en rango . . . . . . . . . . . . . . . . 14

2.3.2. Señal en acimut . . . . . . . . . . . . . . . 16

2.3.3. Señal en banda base . . . . . . . . . . . . . . . . . 19

2.4. Resolución SAR . . . . . . . . . . . . . . . . . . . . . 19

2.4.1. Resolución en rango . . . . . . . . . . . . 20

2.4.2. Resolución en acimut . . . . . . . . . . . . . . 21

2.4.3. Apertura sintética. . . . . . . . . . . . . . 22

2.5. Datos SAR simulados . . . . . . . . . . . . . . . 23

2.6. Enfoque de datos . . . . . . . . . . . . . . . . 23

2.6.1. Algoritmo de Rango-Doppler . . . . . . . . . . . . . . . . 25

2.6.2. Resultados para datos simulados . . . . . . . . . . . 29 
2.6.3. Resultados para datos reales . . . . . . . . . . . . . 30

2.7. Conclusiones . . . . . . . . . . . . . . . . . . . . 31

2.A. Cálculo de la compresión en rango . . . . . . . . . . . . . . 35

2.B. RDA: Implementación en MATLAB . . . . . . . . . . . . . . 37

3. Datos Polarimétricos y Modelos Estadísticos 43

3.1. Datos SAR Polarimétricos . . . . . . . . . . . . . . . . 43

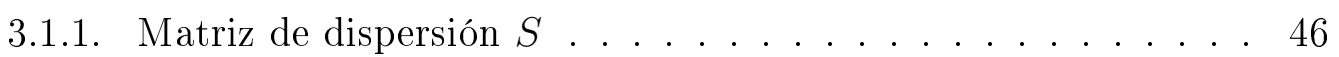

3.1.2. Vector objetivo . . . . . . . . . . . . . . . . 46

3.1.3. Matrices de Coherencia y Covarianza . . . . . . . . . . . . 47

3.1.4. Speckle. . . . . . . . . . . . . . . . . 48

3.2. Modelo para datos homogéneos . . . . . . . . . . . . . . . . 49

3.2.1. Número equivalente de observaciones . . . . . . . . . . . . 50

3.3. Modelo para datos no-homogéneos: la distribucion $\mathcal{G}_{p}^{0}$. . . . . . . . 51

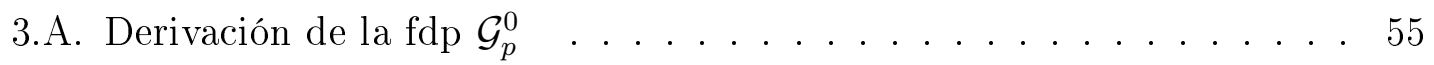

4. Clasificación para datos homogéneos $\quad 57$

4.1. Introducción . . . . . . . . . . . . . . . . . . . 57

4.2. Modelo de Datos . . . . . . . . . . . . . . . . 60

4.3. Estructura del algoritmo . . . . . . . . . . . . . . . . . 61

4.3.1. Inicialización . . . . . . . . . . . . . . . 63

4.3.2. Selección del modelo . . . . . . . . . . . . . . . . . 63

4.3.3. Refinamiento ................. 69

4.3.4. Suavizado . . . . . . . . . . . . . . . . . 71

4.4. Desempeño con datos simulados . . . . . . . . . . . . . . . . 72

4.4.1. Datos Simulados . . . . . . . . . . . . . . . 72

4.4.2. Resultados . . . . . . . . . . . . . . . 73

4.4.3. Desempeño ................... 76

4.5. Desempeño con datos reales . . . . . . . . . . . . . . . . . . 77

4.5.1. Resultados . . . . . . . . . . . . . 77

4.5.2. Desempeño . . . . . . . . . . . . . . . . 80

4.6. Discusión . . . . . . . . . . . . . . . . . . . 81 
4.7. Conclusiones . . . . . . . . . . . . . . . . . . . . 83

5. Clasificación para datos no-homogéneos 87

5.1. Introducción . . . . . . . . . . . . . . . . . . 87

5.2. Modelo de Datos . . . . . . . . . . . . . . . . . . . 88

5.2.1. Modelo de mezclas . . . . . . . . . . . . . . . . . . . . . . . 89

5.3. Estructura del algoritmo . . . . . . . . . . . . . . . . . . . . 89

5.3.1. Inicialización . . . . . . . . . . . . . . . . . . 90

5.3.2. División-fusión . . . . . . . . . . . . . . . . . 90

5.3.3. Estimación . . . . . . . . . . . . . . . . . . . 92

5.3.4. Clasificación . . . . . . . . . . . . . . . . . . . 94

5.3.5. Suavizado . . . . . . . . . . . . . . . . . . 94

5.4. Desempeño con datos simulados . . . . . . . . . . . . . 94

5.4.1. Resultados . . . . . . . . . . . . . . . . . . 96

5.5. Desempeño con datos reales . . . . . . . . . . . . . . . 97

5.5.1. Resultados . . . . . . . . . . . . . . . . . . . . 99

5.6. Conclusiones . . . . . . . . . . . . . . . . . . . . . . 100

5.A. Cálculo de EM para mezcla de densidades $\mathcal{G}_{p}^{0} \quad \ldots \ldots \ldots . \ldots 103$

5.A.1. Cálculo de $Q\left(\boldsymbol{\theta} / \boldsymbol{\theta}^{\prime}\right) \ldots \ldots \ldots 3$

5.A.2. Maximización . . . . . . . . . . . . . . . 105

6. Conclusiones 107

6.1. Trabajo Futuro . . . . . . . . . . . . . . . . . . . 110

$\begin{array}{ll}\text { Bibliografía } & 113\end{array}$ 


\section{Lista de abreviaturas}

BIC Bayesian Information Criterion - Criterio de Información Bayesiano, p.6

CEM Classification-Expectation-Maximization-Clasificación-Esperanza-Maximización, p. 4

ECM Expectation Conditional Maximization - Esperanza Condicional Maximización, p.92

EM Expectation-Maximization - Esperanza-Maximización, p.3

ENL Equivalent Number of Looks- Número Equivalente de Observaciones, p.51

FDP función de densidad de probabilidad, p.49

FFT Fast Fourier Transform - Transformada Rápida de Fourier, p.26

GROUND RANGE Rango sobre la superficie terrestre, p.5

IFFT Inverse Fast Fourier Transform - Transformada Rápida de Fourier Inversa, p. 26

JPL Jet Propulsion Laboratories-Laboratorios de propulsión a chorro, p.1

MAP Máximo A Posteriori, p.5

MULTILOOK Observación Múltiple, p.5

NOAA National Oceanic and Atmospheric Administration- Administración Nacional Oceánica y Atmosférica, p.1

PFA Probability of False Alarm - Probabilidad de Falsa Alarma, p.91

PRF Pulse Repetition Frecuency - Tasa de Repetición de Pulsos, p.11

PRI Pulse Repetition Interval - Intervalo de Repetición de Pulsos, p.11

RADAR Radio Detection And Ranging - Detección y Localización por Radio, p.1

SAR Synthetic Aperture Radar - Radar de Apertura Sintética, p.1 
SEM Stochastic Expectation-Maximization- Esperanza-Maximización Estocástico, p. 4

SLC Single Look Complex - Complejos de Observación Única, p.4 


\section{Capítulo 1}

\section{Introducción}

Los inicios del Radar de Apertura Sintética se remontan a principios de la década de 1950, en los Estados Unidos. Surgió ante la necesidad de una herramienta militar para reconocimiento aéreo que fuera funcional bajo cualquier condición climática. Dada la reconocida propiedad de penetrar las nubes y niebla, y no depender de la luz solar para su funcionamiento, el radar parecía ser la elección adecuada. Sin embargo, las dimensiones de la antena requerida para obtener una imagen de resolución adecuada hacían inviable su montaje en cualquier aeronave. En 1951, el matemático Carl Wiley, que trabajaba para Goodyear Aircraft Company, propuso la idea que cada objeto iluminado por el radar, tendría una velocidad relativa diferente respecto de la antena a medida que ésta se desplaza, definiendo su propio corrimiento Doppler. Sugirió que procesando la frecuencia de la señal recibida a lo largo de la adquisición, sería posible formar imágenes detalladas, que requerirían antenas mucho más grandes con un radar convencional.

De manera contemporánea e independiente de Wiley, un grupo de investigadores de la Universidad de Illinois desarrollaron la misma idea y presentaron en 1952 el algoritmo "Doppler Beam Sharpening" que explotaba el concepto del procesamiento Doppler. En base a estas ideas, en 1957 un SAR aerotransportado produjo los primeros resultados de relevancia, basado en procesamiento óptico de los datos, en

manos de Emmert Leith, de la Universidad de Michigan. En 1974, la NOAA junto con ingenieros el JPL comenzaron a explorar la posibilidad del la observación del 
océano con un SAR montado en un satélite. Esto condujo a que en 1978 se produjera el lanzamiento del Seasat, el primer SAR satelital de uso civil. A partir de entonces, la utilización del SAR no ha cesado de crecer proveyendo una invaluable herramienta para la observación de la Tierra. En la actualidad, se consiguen resoluciones de decenas de centímetros a decenas de kilómetros de distancia. Tiene aplicación en las más diversas disciplinas, como observación de hielos, medición de glaciares, lluvias, erosión, estructura vegetal, geología y desastres naturales, entre otras.

El Radar de Apertura Sintética es un sistema radar que es utilizado para formar imágenes. Es un sistema móvil, montado en un aeroplano o satélite que funciona emitiendo ondas electromagnéticas y recibiendo las reflexiones que se producen en la superficie bajo estudio. Estas reflexiones proveen la información del terreno que es recuperada mediante una conjunción de técnicas de procesamiento analógico-digital y procesamiento estadístico de señales. La característica que diferencia al SAR del radar convencional es su resolución en acimut. Mientras que en los sistemas convencionales este parámetro viene determinado por el ancho de haz de la antena y la distancia al objetivo, para los sistemas SAR la resolución en acimut está determinada por el procesamiento Doppler de la señal recibida. A medida que la antena se desplaza recibe las reflexiones del objetivo durante todo el tiempo que el mismo permanece iluminado por el haz. La combinación de esta información logra el efecto de una apertura de antena "sintética" mucho mayor que la apertura física, logrando una resolución en acimut del orden de las dimensiones físicas de la antena y virtualmente independiente del rango. Esta propiedad es la que le da el nombre al sistema SAR.

Una característica distintiva del SAR es que trata con objetivos distribuidos, es decir, objetivos cuya extensión es mayor a su celda de resolución. Debido a esto, el concepto de sección transversal de radar es redefinido a una sección transversal por unidad de área, que representa la energía reflejada por el objetivo para cada polarización. Por otro lado, el hecho de que la información se obtiene a partir de la interacción coherente de las ondas electromagnéticas con la superficie, determina que las imágenes obtenidas sean afectadas por el efecto speckle. Estas características hacen que los modelos basados en ruido aditivo y estadística puramente gaussiana 
sean inadecuados en la generalidad de los casos. Los modelos que describen más fielmente la señal de retorno son de estructura multiplicativa.

Las características mencionadas en el párrafo anterior serán tratadas en la tesis con el objetivo de desarrollar algoritmos de clasificación de datos, que es la rama del procesamiento SAR donde el presente trabajo realiza el principal aporte. En primer lugar, se considerará el caso de datos denominados homogéneos, donde el modelo multiplicativo general se simplifica a un modelo gaussiano complejo multivariante para los datos SLC. Se propone un algoritmo iterativo basado de mezcla de densidades gaussianas y el método EM, con capacidad para determinar la cantidad de clases presentes en la imagen. Luego, se considera el caso más general de objetivos no homogéneos, donde el comportamiento estadístico difiere del gaussiano y es necesario utilizar el modelo multiplicativo. En ese caso, se utiliza una mezcla de densidades $\mathcal{G}_{p}^{0}$, capaz de describir adecuadamente datos con textura, provenientes de objetivos heterogéneos y muy heterogéneos.

Hasta donde se tiene conocimiento, la determinación de la cantidad de clases presentes en una imagen (que establece la cantidad de parámetros a estimar), no ha sido un aspecto suficientemente considerado por los algoritmos de clasificación clásicos, basados en características polarimétricas. En los algoritmos desarrollados se propone una instancia de selección de modelo que asegura la mínima complejidad y determina el número de clases presentes en la imagen.

Cabe mencionar que si bien este proyecto se enfoca en el problema SAR, los algoritmos desarrollados pueden adaptarse a otras áreas, como imágenes biomédicas y radar meteorológico.

\subsection{Resultados preexistentes}

La clasificación de datos SAR se divide principalmente en dos enfoques diferentes. Uno basado en técnicas de procesamiento de señal, y otra donde se explotan características específicas de los datos SAR polarimétricos. Entre estos últimos, en van Zyl (1989) se propone una clasificación basada en la dispersión con rebotes pares, rebotes impares y dispersión difusa, estableciendo una clasificación en tres clases 
diferentes. En Cloude y Pottier (1997) se propone un método de clasificación no supervisado basado en la descomposición del vector objetivo. Esta descomposición permite agrupar los píxeles de acuerdo al proceso principal de reflexión (superficial, dipolo o múltiple) y el grado de aleatoriedad (entropía). Con este enfoque se extendió el número de clases a nueve.

Para los algoritmos basados en la distribuciones de probabilidad de los datos polarimétricos, el procedimiento usual es definir una distancia estadística y asignar los píxeles a cada clase de manera de minimizar dicha distancia. En Kong et al. (1988) se deriva una distancia basada en el modelo gaussiano complejo de la matriz de dispersión y en Yueh et al. (1993) se extiende este modelo para datos polarimétricos normalizados. Para datos con procesamiento multilook, el modelo gaussiano ya no es válido y debe recurrirse a la distribución Wishart de la matriz de covarianza de los datos. En Lee et al. (1999) se desarrolla un método no supervisado basado en dicha distribución que usa el esquema propuesto en Cloude y Pottier (1997) como inicialización y una clasificación iterativa.

Más recientemente, métodos iterativos basados en el algoritmo EM (Dempster et al., 1977) han sido propuestos para el problema de clasificación. En Kayabol y Zerubia (2013) se propone un método no supervisado basado en CEM, donde se utiliza un modelo de mezclas para describir la amplitud y textura de los datos. Por otro lado, en Yuan et al. (2006) y Dutta y Sarma (2014) se usa EM con un criterio de clasificación MAP con un modelo de mezcla de gaussianas para datos de amplitud. En Horta et al. (2008b) el método SEM es usado junto con la distribución $\mathcal{G}_{p}^{0}$ (Freitas et al., 2005), para describir terrenos homogéneos, heterogéneos y muy heterogéneos respecto del grado de textura.

\subsection{Contribuciones originales}

Las contribuciones originales de esta tesis se relacionan con la clasificación de datos SAR polarimétricos. En primer lugar, se desarrolla un algoritmo de clasificación para imágenes SLC. Este tipo de datos posee un bajo nivel de pre-procesamiento. Pueden presentarse calibrados radiométricamente o geométricamente para ser re- 
presentados en Ground Range, pero no poseen un procesamiento multilook para disminuir el efecto speckle. Esto produce una imagen ruidosa, pero con la máxima resolución espacial posible. Por este motivo, la segmentación de datos producida en base a la clasificación de datos SLC conserva teóricamente la máxima resolución alcanzable. En base a esta idea, y considerando que el modelo gaussiano multivariante es válido para el vector de objetivo (es decir, cuando se trata de datos homogéneos), se asignó al conjunto de datos SLC un modelo de mezcla (suma ponderada) de densidades gaussianas complejas multivariantes, donde cada componente de la mezcla constituye una clase a identificar. Cada clase está determinada por la media y matriz de covarianza de la densidad gaussiana, y por el peso de su contribución en la mezcla. Este tipo de modelo mantiene estructuralmente la simpleza de la densidad gaussiana, a la vez que, por efecto de la mezcla, consigue representar realizaciones que una única densidad gaussiana no puede describir adecuadamente.

Para realizar la clasificación se propone un criterio MAP, para lo cual es necesario estimar los parámetros de cada clase (peso, media y matriz de covarianza), y asignar cada observación (píxel) a la clase que presente mayor verosimilitud. Debido al modelo de mezcla, la estimación de los parámetros por el método de máxima verosimilitud no es sencilla y no posee expresión analítica cerrada. Por ello se adoptó el algoritmo Expectation-Maximization, que resuelve la estimación de máxima verosimilitud de forma iterativa trabajando con funciones más simples que poseen expresión cerrada para el caso gaussiano. En base a este enfoque se desarrolló un algoritmo que fue aplicado a datos simulados y reales. Los resultados fueron presentados en el congreso ArgenCon 2014 y luego publicados en la revista IEEE Latin America Transactions (Fernandez Michelli et al., 2014).

Conjuntamente con lo anterior, las ideas del modelo de mezcla y el algoritmo EM se aplicaron a datos SAR de amplitud descriptos por el modelo GARCH (Noiboar y Cohen, 2007). Este tipo de proceso no estacionario se caracteriza por la variación temporal (espacial, para el caso de imagen SAR) de su varianza, de forma autorregresiva. Esto permite modelar datos provenientes de densidades con colas pesadas y describir procesos con estadística más compleja que la gaussiana. Como contraparte, los procesos GARCH no poseen expresión analítica para su densidad, lo que obliga 
a trabajar con densidades y varianzas condicionales. Si bien en esta tesis no se desarrolla esta línea de trabajo, la aplicación de modelos GARCH a datos SAR produjo buenos resultados que fueron presentados en ArgenCon 2014, y luego publicados en la revista IEEE Latin America Transactions (Pascual et al., 2014).

El clasificador desarrollado para imágenes SLC mostró ser robusto ante su inicialización, siempre que los datos se mantengan poco texturados y el modelo gaussiano aplique. Esto permitió implementar una inicialización sencilla y no exigente que no requiere datos previos del terreno. Sin embargo, la cantidad de clases que el algoritmo debía identificar tenía que ser especificada. En vista de esto, se continuó con la línea de trabajo para producir un clasificador completamente no supervisado, que no requiera de información previa del terreno ni la especificación del número de clases presentes.

En base al Criterio de Información Bayesiano (BIC), se introdujo una instancia de selección del número de clases, evaluando la complejidad del modelo. Conjuntamente se implementó un instancia de fusión de clases, basada en la distancia de Kullback-Leibler, que permite reducir iterativamente el número de clases, sin alterar significativamente la estructura de datos inicial. El funcionamiento conjunto de estas dos etapas permite la identificación automática del número óptimo de clases desde el punto de vista de complejidad del modelo. Este nuevo algoritmo se aplicó a datos simulados y reales con muy buenos resultados. Este trabajo está aceptado para su publicación en la revista Journal of Photogrammetry and Remote Sensing de Elsevier.

Finalmente, la estructura del algoritmo, la versatilidad del modelo de mezclas y el esquema de método EM se extendieron al caso más general de datos no-homogéneos. La presencia de textura en los datos implica un alejamiento de la estadística gaussiana y obliga a utilizar un modelo más complejo. En este contexto, el modelo más utilizado para la señal de retorno es el multiplicativo, donde el dato SAR polarimétrico se expresa cómo el producto entre una variable aleatoria se relaciona con la textura y la matriz de covarianza que da cuenta de la característica polarimétrica del objetivo (Lee y Pottier, 2009, Ch.4). El algoritmo desarrollado se adaptó a este nuevo modelo, utilizando mezclas de densidades $\mathcal{G}_{p}^{0}$. Se obtuvieron excelentes resultados 
para datos simulados y reales. El trabajo correspondiente para una versión supervisada del algoritmo fue presentado en RPIC en octubre de 2015 (Fernández Michelli et al., 2015).

La versión no supervisada requirió una estrategia diferente para la selección del número de clases debido a la complejidad de las densidades involucradas, basada en test de hipótesis y una estrategia top-down. Se planea enviar el artículo correspondiente a una revista internacional del área para su publicación en febrero de 2016 (probablemente a IEEE Signal Processing Letters).

\subsection{Organización de la tesis}

En el Cap. 2 se presentan las generalidades de un sistema SAR. Se estudia la geometría del problema, se definen los parámetros de relevancia y se establece la expresión de la señal recibida en función de ellos. Se aborda el concepto de apertura sintética que caracteriza la resolución del sistema y lo diferencia del radar convencional. Luego se estudia el proceso de enfoque a partir de los datos demodulados (datos crudos). Finalmente se explica este proceso a través del algoritmo de enfoque RDA y se presentan los resultados de su implementación en datos reales.

En el Cap. 3 se describen los datos polarimétricos característicos del sistema SAR en los que se basa todo el proceso de formación de imágenes y extracción de la información. Se presenta la descripción polarimétrica de los datos a través de la matriz de dispersión $\mathbf{S}$ y su representación a través de los vectores objetivo. Se definen las matrices de coherencia y covarianza, que representan adecuadamente los datos provenientes de objetivos extendidos y constituyen la representación básica para el procesamiento estadístico. Luego se estudian los modelos estadísticos que describen a los datos polarimétricos. Se introduce el concepto de speckle y su efecto en las imágenes SAR. Se trata el modelo multiplicativo como estructura general de la señal de retorno SAR. Se presenta el modelo Wishart para la matriz de covarianza de los datos, que contiene al información polarimétrica del objetivo subyacente y los diferentes modelos para la retrodispersión, que contiene la información de tex- 
tura del terreno. Finalmente se presenta el modelo $\mathcal{G}_{p}^{0}$ para la señal de retorno y su versatilidad para describir a los datos SAR provenientes de diferentes terrenos.

El resto de los capítulos se dedica a la clasificación de datos y constituye el principal aporte de esta tesis. El Cap. 4 aborda la clasificación de imágenes SAR, presentando la versión del algoritmo para datos homogéneos donde el modelo gaussiano multivariante es válido para el vector objetivo. Luego, en el Cap. 5 se presenta la versión generalizada para terrenos no-homogéneos donde se utiliza el modelo $\mathcal{G}_{p}^{0}$ como base para incluir la naturaleza multiplicativa y texturada de los datos. Ambos algoritmos se basan fuertemente en el método EM y sus derivados, por lo que se presentará el sustento teórico del mismo y su aplicación para cada caso. Además, se estudiará el Criterio de Información Bayesiana (BIC) como herramienta para evaluar la complejidad del modelo y su utilización para determinar el número óptimo de clases presentes en la imagen. Ambos algoritmos serán aplicados a datos simulados (generados con los modelos propuestos) y a datos SAR reales. Los resultados obtenidos y el desempeño de la clasificación serán presentados hacia el final de los capítulos correspondientes.

Finalmente se concluye la tesis con el Cap. 6 donde se discuten los resultados obtenidos y las posibles líneas de trabajo futuro. 


\section{Capítulo 2}

\section{Radar de Apertura Sintética}

Un sistema SAR se basa en un radar móvil que ilumina la superficie terrestre formando imágenes para su estudio. A lo largo de su trayectoria, la antena colocada sobre la plataforma móvil emite continuamente pulsos de microondas y recibe las reflexiones producidas en la superficie. Estas reflexiones son grabadas y luego procesadas para formar la imágenes de la escena. En la geometría SAR se distinguen dos direcciones principales: la dirección perpendicular al movimiento del sensor -rangoy la dirección paralela al movimiento de éste denominada acimut. Cada una de estas dimensiones se vincula con la correspondiente imagen SAR bidimensional, de manera que cada punto de la superficie es mapeado a un punto de la imagen. En este capítulo se explicará el proceso de la formación de la imagen SAR a partir del procesamiento de las reflexiones recibidas, partiendo de la geometría del problema y del modelo de señal de microondas emitida. Se abordará sólo el caso de la modalidad stripmap aerotransportado donde la antena apunta en dirección perpendicular a la trayectoria.

Se estudiará la señal recibida y los parámetros que la describen, así como la conformación de los datos crudos, que son los datos de más bajo nivel de procesamiento. Posteriormente, se estudiará el proceso de formación de la imagen a partir de dichos datos denominado enfoque, y hacia el final del capítulo se presentará una implementación del algoritmo RDA para esa tarea, aplicado a datos de la misión 
$\mathrm{SARAT}^{1}$. Un estudio más completo que el presentado aquí se encuentra en los trabajos de Cumming y Wong (2005) y Wang (2008) tanto sobre los algoritmos de enfoque como el tratamiento de otras modalidades SAR.

\subsection{Geometría del SAR aerotransportado}

La Fig. 2.1 muestra la geometría básica de un sistema SAR aerotransportado. El radar se desplaza a una velocidad $V r$ a una altura $H$ sobre al superficie en línea recta. La antena está orientada de manera que su haz apunta hacia la superficie en dirección perpendicular a la trayectoria del aeroplano. La porción del terreno desde la cual se refleja el pulso de radar se denomina huella, y está determinada por el patrón de haz de la antena y la geometría del terreno. La concatenación de huellas de la antena a lo largo de la trayectoria del radar determina una franja de terreno denominada franja de haz. El ángulo $\theta_{i}$ que separa la dirección del centro de haz de la dirección del nadir se denomina ángulo de incidencia.

Se definen dos direcciones principales en la geometría presentada. La dirección de acimut, que coincide con la dirección de desplazamiento del radar, y la dirección de rango, para la cual deben distinguirse dos casos: rango inclinado (Slant Range) y el rango sobre el suelo (Ground Range). El primero es la distancia que separa a la antena del objetivo, mientras que el segundo es la distancia desde el nadir de la antena hasta el objetivo, medida sobre la superficie terrestre. Denominaremos $R_{0}$ a la distancia entre la antena y el centro de la huella del haz medida sobre el rango inclinado.

Asociado a cada una de estas direcciones se definen dos escalas temporales: el tiempo rápido $\tau$, que es el tiempo de propagación del pulso a lo largo del rango inclinado y se relaciona con él mediante la velocidad de la luz $c$, y el tiempo lento $\eta$ (o tiempo de acimut) que es el tiempo que toma un desplazamiento en la dirección de acimut (las denominaciones rápido y lento surgen de la comparación entre las velocidades asociadas a cada dimensión). La relación entre el tiempo lento y el desplazamiento en acimut está dada por la velocidad $V_{r}$. Dada la relación lineal que

\footnotetext{
${ }^{1}$ http://www.conae.gov.ar/index.php/espanol/misiones-satelitales/saocom/sarat
} 


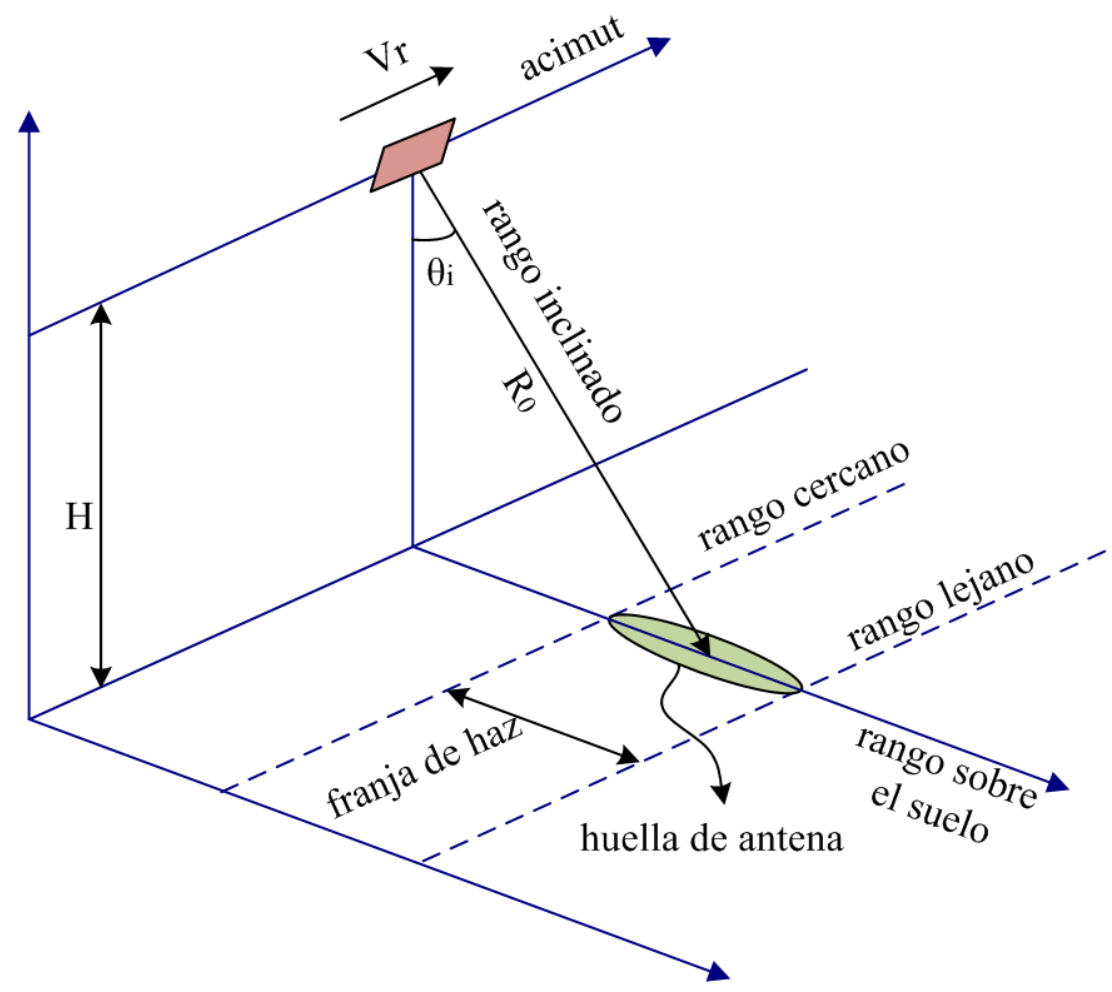

Figura 2.1: Geometría SAR aerotrasnportado.

existe entre las dimensiones de distancia y tiempo, la señal SAR puede representarse indistintamente con cualquiera de las dos como variables independientes.

El radar emite pulsos de microondas sucesivos de duración $T_{r}$ a una tasa denominada PRF, y cuyo período se denomina PRI. Luego de la transmisión de un pulso, el radar cambia a la modalidad de recepción para recibir las reflexiones hasta la transmisión de un nuevo pulso como se muestra en la Fig. 2.2. Cada tiempo de recepción corresponde a una posición distinta del radar en dirección de acimut, y por lo tanto, a una posición diferente de la huella de la antena. De esta manera, se colecta toda la información a lo largo de la franja de haz.

\subsection{Arreglo de datos}

La señal recibida por el radar puede interpretarse como una señal unidimensional mediante la concatenación de la respuestas a los sucesivos pulsos. Sin embargo, 


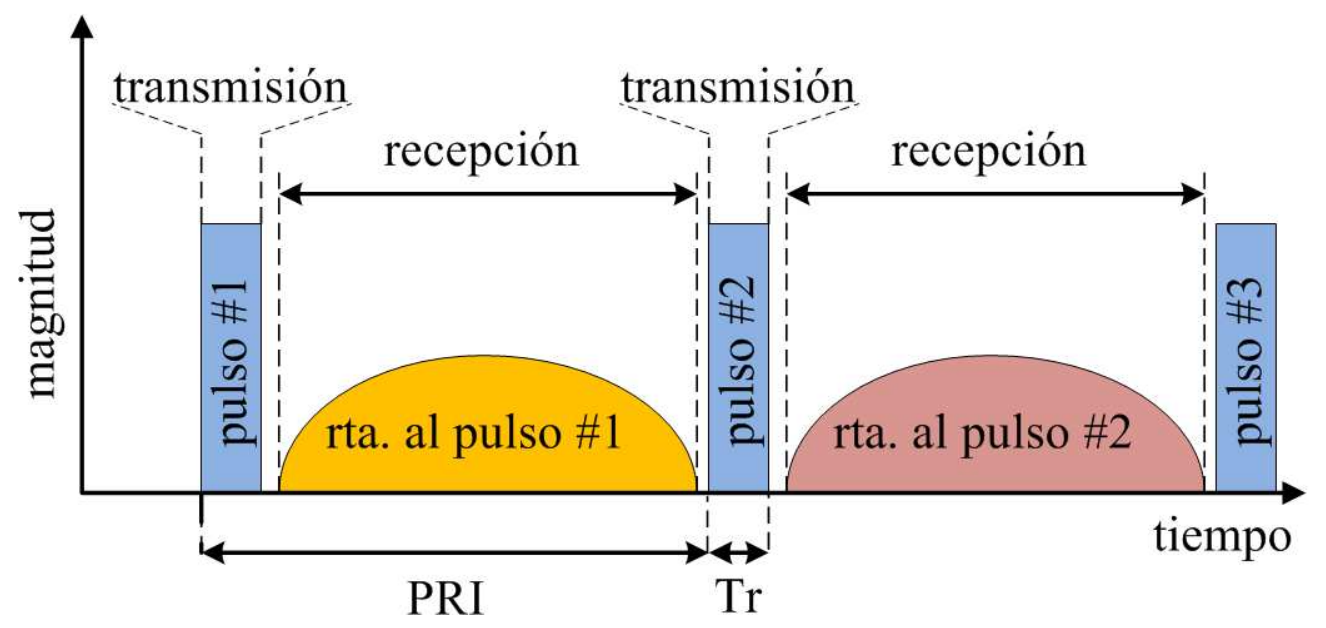

Figura 2.2: Ventanas de transmisión y recepción.

resulta mucho más apropiado entenderla como una señal bidimensional, dada la correspondencia entre la geometría bidimensional del problema (rango y acimut) y la imagen 2D de la superficie.

Se tomará como referencia la Fig. 2.3. Conforme el radar se desplaza en acimut, recibe los ecos provenientes de cada posición de la huella de antena sobre el terreno. La señal proveniente de cada uno de estos pulsos es demodulada, muestreada y guardada en una fila de memoria, de manera que cada fila de la matriz de datos formada corresponde al eco de una posición de acimut. De la misma forma, cada columna corresponde a una posición de rango determinada. El intervalo de tiempo entre muestras adyacentes de una fila corresponde al período de muestreo de la señal. El intervalo de tiempo entre muestras adyacentes de una columna es igual al PRI. La primera columna del arreglo contiene la información correspondiente al rango cercano y la última contiene la información del rango lejano. De esta manera queda conformado un arreglo de datos bidimensional, donde la dimensión vertical corresponde a acimut (o al tiempo lento) y la horizontal al rango (tiempo rápido o retardo). 


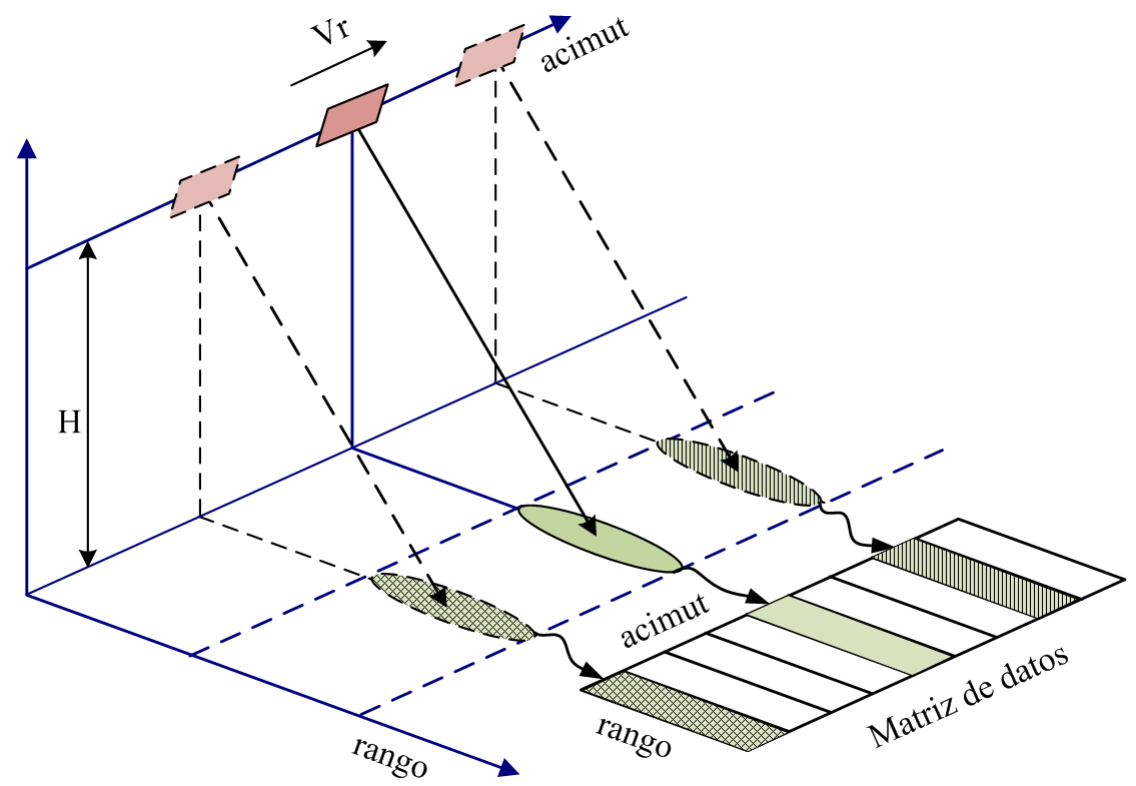

Figura 2.3: Construcción de la matriz de datos

\subsection{Señales SAR}

Para comprender la naturaleza de las señales que caracterizan al sistema SAR, debemos en primer lugar entender la relación entre la distancia en rango inclinado y el tiempo en acimut. En la Fig. 2.4 se muestra una representación simplificada de la geometría donde el eje vertical corresponde al rango inclinado y el horizontal al acimut. La distancia desde la antena al objetivo resulta

$$
R(\eta)^{2}=\left(V_{r} \eta\right)^{2}+R_{0}^{2},
$$

donde tiempo de acimut $\eta$ está referido al instante en que la plataforma pasa por el punto más cercano al objetivo. Se observa que (2.1) describe una relación hiperbólica entre $R$ y $\eta$, denominada Ecuación de Rango, que tendrá profundas consecuencias en la señal recibida y en el algoritmo de enfoque. 


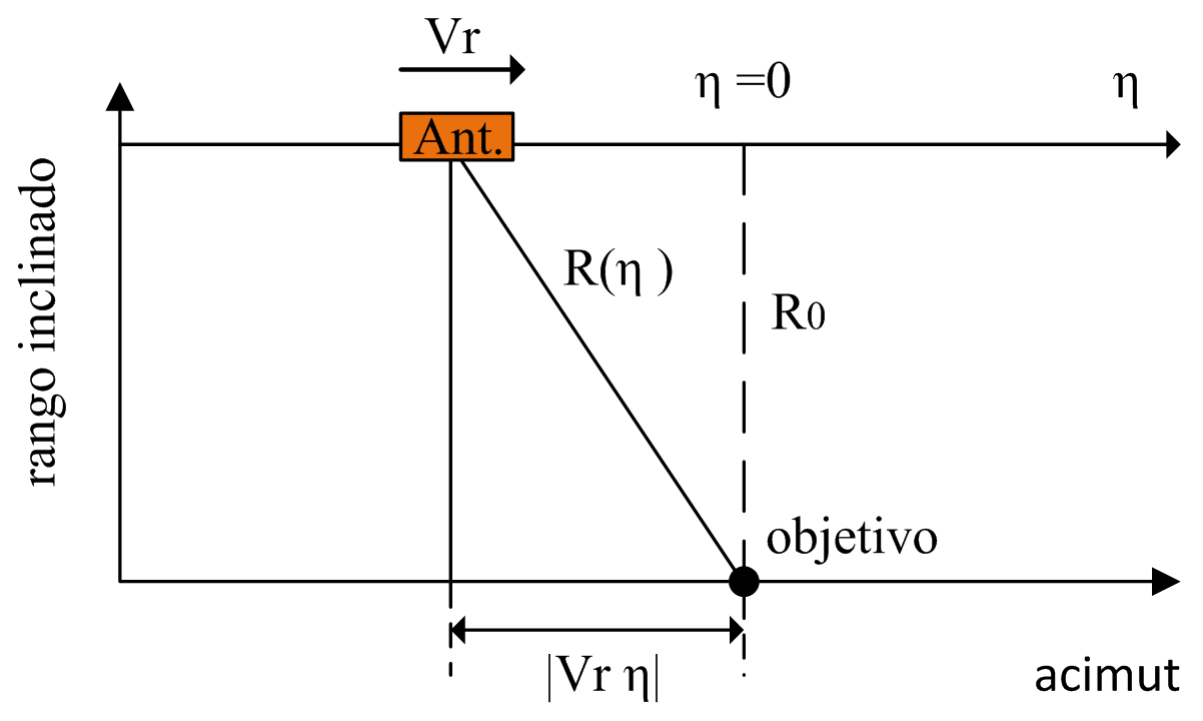

Figura 2.4: Esquema de la ecuación de rango

\subsubsection{Señal en rango}

La forma usual del pulso radar utilizado en SAR corresponde a una señal chirp de duración $\operatorname{Tr}$ :

$$
s_{\text {pulso }}(\tau)=w_{r}(\tau) \cos \left(2 \pi f_{0} \tau+\pi K_{r} \tau^{2}\right),
$$

donde $f_{0}$ es la frecuencia de portadora, $K_{r}$ es la tasa de modulación de frecuencia y $w_{r}$ es la forma del pulso. Para este desarrollo, supondremos un pulso rectangular, por lo que $w_{r}(\tau)=\operatorname{rect}\left(\tau / T_{r}\right)$. La frecuencia instantánea del pulso $s_{p u l s o}$ varía linealmente con el tiempo durante el intervalo $T_{r}$ a una tasa dada por $K_{r}$, de manera que la máxima frecuencia instantánea viene dada por $f_{\max }=f_{0}+\left|K_{r}\right| T_{r} / 2$. Cuando la tasa $K_{r}$ es positiva, al pulso se lo denomina "up-chirp", indicando una frecuencia creciente. En caso contrario, se lo denomina "down-chirp".

El ancho de banda ocupado por el pulso es de suma importancia en la determinación de la resolución SAR en rango. Dado que la señal chirp puede considerarse como un señal sinusoidal que incrementa su frecuencia con el tiempo, el ancho de banda ocupado por la misma puede determinarse a partir de su duración y de su tasa de modulación: $B W_{\text {pulso }}=K_{r} T_{r}$. 
La Fig. 2.5 esquematiza cómo se genera la señal en rango. El pulso de duración $T_{r}$ se propaga desde la antena hacia el terreno. Luego de un tiempo $t_{n e a r}$ el frente del pulso incide en la superficie en el rango cercano. En el instante $t_{f a r}$ el flanco final del pulso incide en el punto de rango lejano. De esta manera todo los puntos de la superficie entre el rango cercano y rango lejano son iluminados por el pulso.

La señal reflejada está determinada por la convolución entre el pulso incidente y la reflectividad del area del suelo iluminada, que en el caso general es función de la superficie. Para el presente desarrollo, supondremos en todo momento que tratamos con objetivos puntuales, de manera que el pulso sólo es modificado en amplitud por el objetivo. Esto es equivalente a pensar que la función de reflectividad del mismo es un delta de Dirac de área igual a su valor de reflectividad.

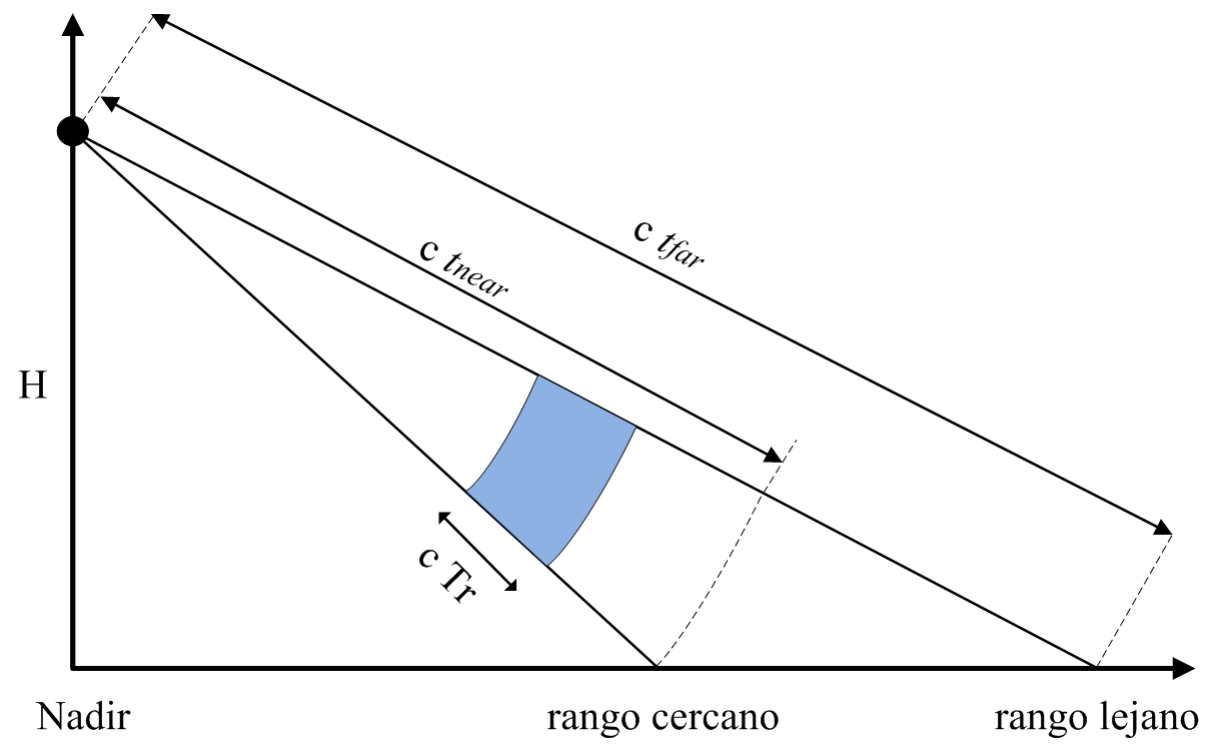

Figura 2.5: Generación de señal en rango

La señal reflejada retorna a la antena en el intervalo de tiempo entre $2 t_{\text {near }} \mathrm{y}$ $2 t_{f a r}$, para ser demodulada, luego muestreada y almacenada. La tasa de muestreo queda determinada por el ancho banda de la señal demodulada, según el criterio de Nyquist. La mínima tasa de muestreo resulta $f_{m r_{\text {min }}}=B W_{\text {pulso }}=K_{r} T_{r}$. Cabe mencionar que la distancia entre el rango cercano y el lejano está determinada por el ancho de haz de la antena en dirección de rango, para un altura determinada. Debe tenerse en cuenta que si el haz es demasiado ancho en esta dirección en relación al 
PRI, la respuesta del rango lejano a un pulso determinado podría arribar a la antena luego de la emisión del pulso siguiente, originando problemas de ambigüedad. Esto impone una restricción para la elección del PRI en el diseño del sistema.

Considerando un objetivo puntual a distancia $R_{o b j}$ del objetivo con reflectividad $A_{o b j}$, la señal recibida por el radar es:

$$
\begin{aligned}
s_{r}(\tau) & =A_{o b j} s_{\text {pulso }}\left(\tau-2 R_{o b j} / c\right) \\
& =A_{o b j} w_{r}\left(\tau-\frac{2 R_{o b j}}{c}\right) \cos \left\{2 \pi f_{0}\left(\tau-\frac{2 R_{o b j}}{c}\right)+\pi K_{r}\left(\tau-\frac{2 R_{o b j}}{c}\right)^{2}\right\},
\end{aligned}
$$

donde $2 R_{o b j} / c$ es el retardo de ida y vuelta de la señal hasta el objetivo. Se observa que la señal posee una componente de frecuencia $f_{0}$ que es la frecuencia de portadora, que será removida en el proceso de demodulación. Posee también un retardo dado por el tiempo de viaje de la señal y conserva la característica de fase cuadrática del pulso incidente. En la expresión (2.3) no se ha tenido en cuenta el cambio de fase que podría introducir el objetivo, ya que es irrelevante para el presente análisis.

\subsubsection{Señal en acimut}

En este apartado se pondrá en evidencia la dependencia de la señal recibida con la posición en acimut. Para ello debe analizarse el efecto Doppler en la señal y definirse los parámetros relacionados con él.

En el caso general, el efecto Doppler es la variación de frecuencia de una onda debido a la velocidad relativa entre la fuente y el receptor. Una onda electromagnética de frecuencia $f_{0}$ que incide sobre un objeto que se está acercando a la fuente, produce una onda reflejada de frecuencia mayor a $f_{0}$. Si el objeto se está alejando, la frecuencia de la onda reflejada es menor a $f_{0}$. A estas variaciones de frecuencia respecto de la portadora se las denomina desplazamiento Doppler. Para el caso SAR, la distancia entre un objeto iluminado y la antena varía según la ecuación de rango (2.1) a medida que el radar se desplaza en acimut. Como puede verse en la Fig. 2.6, el objeto comenzará a ser registrado por el radar en la posición $P_{1}$ y dejará de ser registrado cuando el radar esté en la posición $P_{3}$, siendo $P_{0}$ el punto de mayor acer- 
camiento y por lo tanto donde la señal tendrá mayor intensidad. Entre los puntos $P_{1}$ y $P_{3}$ existe una variación de la distancia entre la fuente (objeto reflector) y el receptor (antena) a medida que transcurre el tiempo lento $\eta$, lo que determina una variación de velocidad relativa entre ambos y el consecuente efecto Doppler. Entre los puntos $P_{1}$ y $P_{0}$ el radar se acerca al objetivo, reduciendo la distancia desde $R_{1}$ hasta $R_{0}$. Por lo tanto en este tramo la señal experimentará un Doppler positivo y tendrá una frecuencia mayor a la de portadora. Por el contrario, entre los puntos $P_{0}$ y $P_{3}$, el radar se aleja del objetivo y la señal experimentará un Doppler negativo. En el punto $P_{0}$, la velocidad relativa será nula y por lo tanto también lo será el desplazamiento Doppler. Debe notarse que la distancia $P_{3}-P_{1}$ a lo largo de la cual la antena ilumina el objeto reflector, está determinada por el ancho de haz en acimut $\theta_{a c}$. Si definimos el origen del tiempo lento en el instante de Doppler nulo y llamamos $f_{\text {Dop }}$ al desplazamiento Doppler, resulta

$$
f_{\text {Dop }}=-2 \frac{f_{0}}{c} \frac{\partial R(\eta)}{\partial \eta}=-\frac{2}{\lambda} \frac{V_{r}^{2} \eta}{R(\eta)}=\frac{2}{\lambda} V_{r} \operatorname{sen}(\theta(\eta)),
$$

donde $\lambda$ es la longitud de onda correspondiente a $f_{0}$.

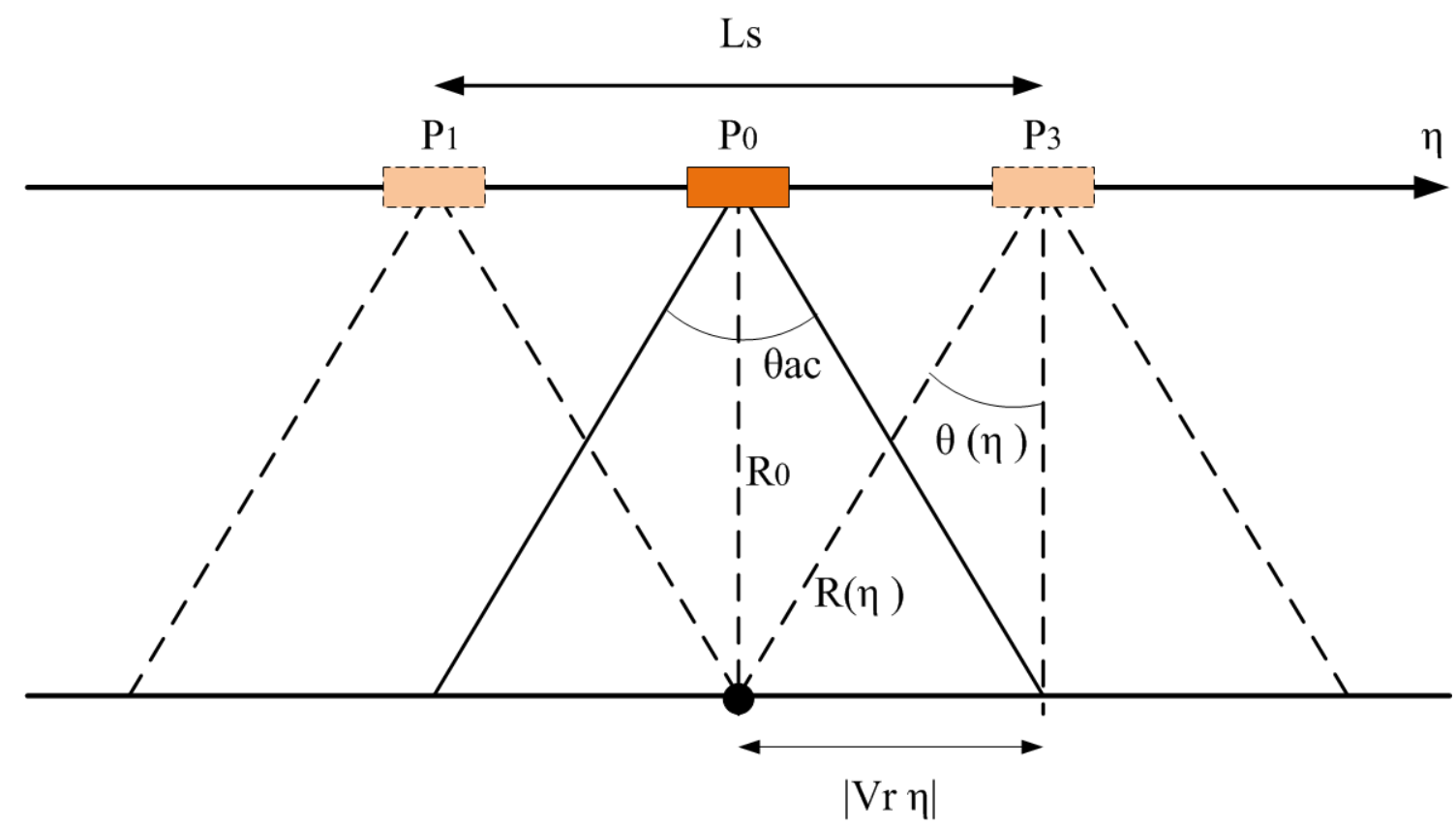

Figura 2.6: Generación de señal en acimut 
El ancho de banda Doppler $\Delta f_{\text {Dop }}$ es el ancho de banda que ocupa la señal debido al desplazamiento Doppler máximo. En base a la Fig. 2.6 es sencillo determinar que $\Delta f_{\text {Dop }}$ resulta de las posiciones $P_{1}$ y $P_{3}$ :

$$
\Delta f_{\text {Dop }}=f_{\text {Dop }}\left(P_{1}\right)-f_{\text {Dop }}\left(P_{3}\right)=2\left(\frac{2}{\lambda} V_{r} \operatorname{sen}\left(\theta_{a c} / 2\right)\right) \simeq \frac{2}{\lambda} V_{r} \theta_{a c},
$$

donde la última aproximación se justifica porque $P_{1}-P_{0} \ll R_{0}$. Teniendo en cuenta que $\theta_{a c}=\lambda / L_{a}$, siendo $L_{a}$ la longitud física de la antena en la dirección de acimut, podemos expresar (2.5) como

$$
\Delta f_{\text {Dop }}=\frac{2 V_{r}}{L_{a}}
$$

En la sección 2.2 se ha establecido que entre dos filas adyacentes del arreglo de datos transcurre un tiempo igual a PRI. De manera que a medida que el radar se desplaza en acimut, la señal es muestreada (observada) en tiempo lento a una tasa PRF. Dado que en esta dimensión la señal tiene un ancho de banda dado por $\Delta f_{\text {Dop }}$, PRF no puede ser menor a $\Delta f_{\text {Dop }}$, lo que establece un limite inferior para este parámetro.

Adicionalmente, se define el Tiempo de exposición $T_{a}$ como el intervalo de tiempo durante el cual el objetivo permanece dentro del ancho de haz, y está dado por

$$
T_{a}=\frac{\left|P_{1}-P_{3}\right|}{V_{r}}=2 \frac{R_{0}}{V_{r}} \tan \left(\theta_{a c} / 2\right) \simeq \frac{R_{0}}{V_{r}} \theta_{a c}=\frac{R_{0} \lambda}{V_{r} L_{a}} .
$$

Otro parámetro de interés es la tasa de modulación de frecuencia en acimut $K_{a}$, que representa la velocidad máxima con que cambia la frecuencia de la señal en acimut, y está dada por

$$
K_{a}=\frac{2}{\lambda}\left[\frac{\partial^{2} R(\eta)}{\partial \eta^{2}}\right]_{\eta=0}=\frac{2 V_{r}^{2}}{\lambda R_{0}}
$$

Se puede obtener $\Delta f_{\text {Dop }}$ de manera alternativa, multiplicando las expresiones (2.7) y (2.8). 
Recordando la expresión (2.3), y en base al análisis anterior, la variable $R_{o b j}$ es dependiente del tiempo de acimut, estableciendo la señal recibida como una función bidimensional

$$
\begin{aligned}
s_{r}(\tau, \eta)= & A_{o b j} w_{r}\left(\tau-\frac{2 R(\eta)}{c}\right) w_{a}(\eta) \times \\
& \cos \left\{2 \pi f_{0}\left(\tau-\frac{2 R(\eta)}{c}\right)+\pi K_{r}\left(\tau-\frac{2 R(\eta)}{c}\right)^{2}\right\},
\end{aligned}
$$

donde el factor $w_{a}(\eta)$ indica la ganancia de la antena en la dirección de acimut. Este factor tiene en cuenta el viaje de ida y vuelta de la onda desde que se emite de la antena.

\subsubsection{Señal en banda base}

La señal $s_{r}$ presentada en el apartado anterior describe la señal recibida en la antena como respuesta a un objetivo puntual. Esta señal es demodulada y luego muestreada para formar el arreglo de datos al cual se le aplicará el procesamiento.

El proceso de demodulación en fase y cuadratura de $s_{r}$ recupera su envolvente compleja pasabajos $s_{0}$ :

$$
\begin{aligned}
s_{0}(\tau, \eta)= & A_{o b j} w_{r}\left(\tau-\frac{2 R(\eta)}{c}\right) w_{a}(\eta) \times \\
& \exp \left\{-j 4 \pi f_{0}\left(\frac{2 R(\eta)}{c}\right)\right\} \exp \left\{j \pi K_{r}\left(\tau-\frac{2 R(\eta)}{c}\right)^{2}\right\} .
\end{aligned}
$$

La señal $s_{0}$ es la que finalmente es almacenada y sus muestras son las que conforman los datos SAR de más bajo nivel, denominados "datos crudos".

\subsection{Resolución SAR}

Una de las características más importantes del SAR, así como la del radar convencional, es su resolución. Informalmente puede decirse que la resolución SAR es la 
capacidad del radar para distinguir dos puntos cercanos en la superficie. Dado que es un sistema bidimensional, se distinguen dos resoluciones: resolución en rango, y resolución en acimut. Para determinar correctamente la resolución debe considerarse la respuesta del SAR luego de recibir la señal de un blanco puntual. Es decir, debe evaluarse la señal luego de ser procesada con el filtro adaptado.

\subsubsection{Resolución en rango}

Para determinar la resolución en rango, se supondrá un blanco puntual sobre la superficie a una distancia en rango $R_{0}$ de tal forma que que el retardo de ida y vuelta que produce es $\tau_{o}=2 R_{0} / c$. Debido a que se analiza la resolución en rango, puede considerarse fijo el tiempo en acimut $\eta=\eta_{0}$. De esta manera, puede expresarse $s_{0}$ en forma simplificada $s_{0}^{\prime}$, reuniendo en una constante $K^{\prime}$ todos los factores que dependen sólo del tiempo en acimut:

$$
s_{0}^{\prime}(\tau)=K^{\prime} w_{r}\left(\tau-\tau_{0}\right) \exp \left\{j \pi K_{r}\left(\tau-\tau_{0}\right)^{2}\right\}
$$

$\operatorname{con} w_{r}(\tau)=\operatorname{rect}\left(\tau / T_{r}\right)$.

La respuesta impulsional del filtro adaptado a $s_{0}^{\prime}(\tau), h_{r}(\tau)$, es la versión reflejada y conjugada del pulso emitido:

$$
h_{r}(\tau)=\operatorname{rect}\left(\frac{\tau}{T_{r}}\right) \exp \left\{-j \pi K_{r} \tau^{2}\right\}
$$

La señal filtrada $s_{f}(\tau)$ resultará de la convolución entre $s_{0}^{\prime}(\tau)$ y $h_{r}(\tau)$. El el apéndice 2.A se muestran los detalles del cálculo. El resultado es una señal sinc centrada en $\tau=\tau_{0}$ :

$$
s_{f}(\tau)=\left\{s_{0}^{\prime} * h_{r}\right\}(\tau)=K^{\prime} T_{r} \operatorname{sinc}\left[K_{r} T_{r}\left(\tau-\tau_{0}\right)\right]
$$


La resolución en rango $\Delta_{r}$ está determinada por la mitad del ancho del lóbulo principal $\rho_{r}=1 / 2 K_{r} T_{r}$, expresada en unidades de distancia ${ }^{2}$ :

$$
\Delta_{r}=\frac{c}{2 K_{r} T_{r}}
$$

Si dos objetivos puntuales estuvieran separados a una distancia menor a $\Delta_{r}$ en rango inclinado, sus respuestas se superpondrían y no sería posible distinguirlos como dos objetivos separados. Debe notarse que el producto $K_{r} T_{r}$ es igual al ancho de banda ocupado por la señal según se analizó en la sección 2.3.1. Por lo tanto, la resolución de una señal chirp es el recíproco de su ancho de banda. Esta característica permitirá estimar la resolución en acimut en el apartado siguiente.

\subsubsection{Resolución en acimut}

El la sección 2.3.2 se determinó la dependencia de la frecuencia de la señal $s_{0}$ con el tiempo de acimut. El desplazamiento del radar origina una modulación en frecuencia dada por el efecto Doppler, cuyo ancho de banda está dado por $\Delta f_{\text {Dop }}$. Siguiendo con el razonamiento del apartado anterior, la resolución en acimut será inversamente proporcional al ancho de banda, luego de que la señal haya sido procesada con el filtro adaptado. Es decir, la resolución temporal en acimut resulta $\rho_{a}=L_{a} / 2 V_{r}$ y expresándola en distancia,

$$
\Delta_{a}=\rho_{a} \cdot V_{r}=L_{a} / 2
$$

La derivación anterior se basa en la suposición de que la señal considerada es una chirp en acimut. Aunque no se ha demostrado efectivamente que dicha señal resulta del procesamiento con el filtro adaptado en acimut, esto será evidente cuando se trate el algoritmo RDA en apartados siguientes. Por otro lado, una deducción formal basada en el modelo electromagnético puede hallarse en Cheney (2001).

La expresión 2.15 indica la característica más sobresaliente de un sistema SAR. La resolución en acimut resulta independiente de la distancia al objetivo y del ancho

\footnotetext{
${ }^{2}$ Alternativamente puede tomarse el ancho de $3 \mathrm{~dB}$ del lóbulo principal, resultando $0,886 \rho_{r}$
} 
ancho de haz de la antena como ocurre en un radar convencional. Sólo depende del largo físico de la antena en la dirección de acimut.

Debe tenerse en cuenta que $\Delta_{a}$ es la máxima resolución alcanzable, que surge de considerar que no se recibe energía más allá del ancho de haz en acimut y que su ganancia es constante. En un sistema la antena no presentará tales características y la resolución se verá degradada.

\subsubsection{Apertura sintética}

El concepto de apertura sintética $L_{s}$ es el que da el nombre al sistema SAR y se explica sencillamente en base a los conceptos desarrollados en la sección 2.3.2.

La resolución en acimut de un radar convencional está dada por el producto entre la distancia al objetivo y el ancho de haz en acimut, $\Delta_{a}=2 R_{0} \operatorname{sen}\left(\theta_{a c} / 2\right) \simeq$ $R_{0} \theta_{a c}=R_{0} \lambda / L_{a}$, donde se observa que la resolución es inversamente proporcional a la apertura real de la antena (o largo de la misma en acimut) $L_{a}$. En el caso SAR, la antena ilumina el objetivo durante todo el tiempo que éste permanezca dentro del ancho de haz. El procesamiento coherente de todos los pulsos que se reciben durante ese tiempo tiene el efecto de aumentar la apertura efectiva de la antena, o equivalentemente, de reducir su ancho de haz, logrando una mayor resolución. En la Fig. 2.6 se observa que la distancia sobre la trayectoria del radar durante la cual el objetivo puntual es iluminado es igual a $|P 1-P 3|$. A esta distancia se la denomina apertura sintética $L_{s}$ y viene dada por

$$
L_{s}=\frac{R_{0} \lambda}{L_{a}}
$$

de manera que ancho de haz sintético resulta $\theta_{s}=\lambda / 2 L_{s}=L_{a} / 2 R_{0}$. Si se multiplica $R_{0}$ por $\theta_{s}$ para determinar la nueva resolución sintética se obtiene la resolución en acimut dada por expresión (2.15). Intuitivamente se puede explicar su independencia con el rango ya que cuanto más alejado está el objetivo, más tiempo será iluminado por la antena, compensando los efectos. 


\subsection{Datos SAR simulados}

En esta sección se presenta la simulación de la señal SAR generada por un blanco puntual. Los parámetros utilizados figuran en la Tabla 2.1 son los correspondientes a la misión SARAT. El código utilizado está basado en la implementación de Schlutz (2009).

En la Fig. 2.7 se observa la escena de un blanco puntual caracterizado por su reflectividad unitaria. La Fig. 2.8 muestra la señal generada. Esta señal bidimensional se denomina espacio de señal SAR.

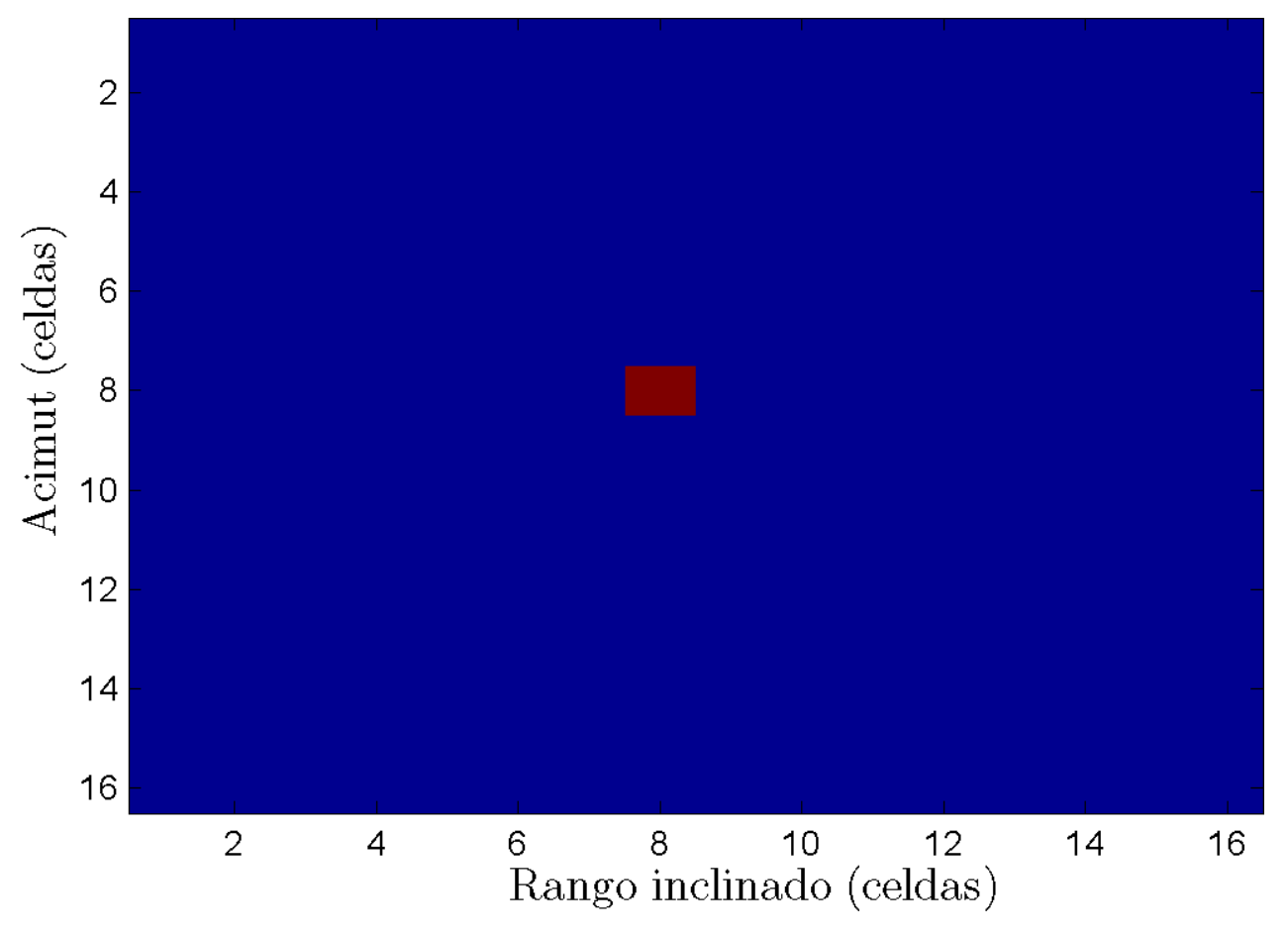

Figura 2.7: Objetivo Puntual.

\subsection{Enfoque de datos}

El propósito de los algoritmos de enfoque de datos SAR es recuperar la función de reflectividad del terreno a partir de la señal recibida. El objetivo es concentrar la energía dispersada por la adquisición SAR en la posición correcta de rango y acimut. 


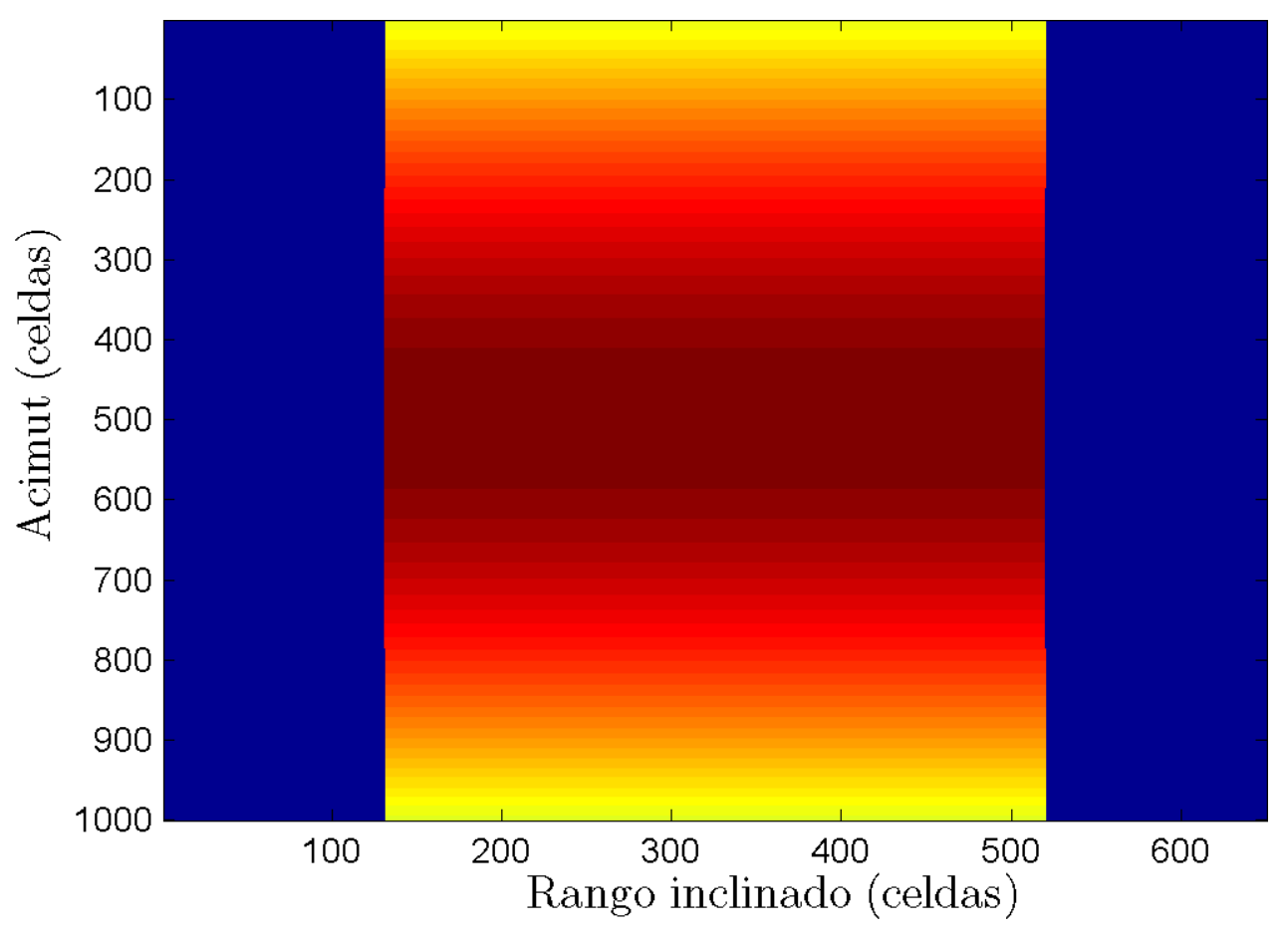

Figura 2.8: Datos SAR Simulados. Espacio de Señal.

En este trabajo se utilizará el algoritmo de Rango-Doppler (RDA) para enfocar los datos simulados y reales. Para ello conviene definir algunos conceptos relacionados con RDA y el espacio de señales sobre el que se aplica.

Para el caso de ancho de haz en acimut pequeño, la expresión hiperbólica (2.1) para el rango puede aproximarse por una parábola. Es decir, si $R_{0} \gg \eta V_{r}$ se puede escribir

$$
R(\eta) \simeq R_{0}+\frac{V_{r}^{2} \eta^{2}}{2 R_{0}} .
$$

Por lo tanto, podemos expresar la señal $s_{0}$ como:

$$
\begin{aligned}
s_{0}(\tau, \eta)= & A_{\text {obj }} w_{r}\left(\tau-\frac{2 R(\eta)}{c}\right) w_{a}(\eta) \times \\
& \exp \left\{-j 4 \pi\left(\frac{R_{0}}{\lambda}\right)\right\} \exp \left\{-j \pi K_{a} \eta^{2}\right\} \exp \left\{j \pi K_{r}\left[\tau-\frac{2 R(\eta)}{c}\right]^{2}\right\},
\end{aligned}
$$


Tabla 2.1: Parámetros de la misión SARAT.

\begin{tabular}{|c|c|c|}
\hline Parámetro & Símbolo & Valor \\
\hline Altura de la plataforma & $H$ & $5375 \mathrm{~m}$ \\
\hline Velocidad de la plataforma & $V_{r}$ & $108 \mathrm{~m} / \mathrm{s}$ \\
\hline Frecuencia de portadora & $f_{0}$ & $1300 \mathrm{MHz}$ \\
\hline Largo del pulso & $T_{r}$ & $10 \mu \mathrm{s}$ \\
\hline Frecuencia de muestreo & $f_{m}$ & $50 \mathrm{MHz}$ \\
\hline Frecuencia de Repetición de Pulso & $\mathrm{PRF}$ & $250 \mathrm{~Hz}$ \\
\hline Velocidad de la luz & $\mathrm{c}$ & $299.792 .458 \mathrm{~m} / \mathrm{s}$ \\
\hline
\end{tabular}

donde $K_{a}$ es la tasa de modulación de frecuencia en acimut calculada previamente. La expresión anterior pone en evidencia la variación cuadrática de la fase en la dirección del tiempo lento, lo que determina una señal chirp en acimut. En el proceso de enfoque las variaciones cuadráticas de la fase con los tiempos de rango y acimut serán removidas mediante la aplicación de filtros adaptados.

Debe observarse que el término $2 R(\eta) / c$ dentro del argumento de $w_{r}$ establece la interdependencia que existe entre tiempo rápido y tiempo lento en la señal. A lo largo del trayecto en acimut, la posición de un objetivo puntual ubicado en $(\tau, \eta)=\left(R_{0}, \eta_{0}\right)$ describirá una trayectoria parabólica en el espacio de señal, de manera que para la posición de acimut genérica $\eta$, la energía del eco estará centrada en $R(\eta)$ y no en $R_{0}$. A este desplazamiento en celdas de rango en función del tiempo de acimut se lo denomina migración de celdas de rango (RCM) y es una característica distintiva de la señal SAR. Para lograr un enfoque exitoso, esta migración debe ser corregida evitando dispersar la energía en celdas de rango adyacentes.

\subsubsection{Algoritmo de Rango-Doppler}

El Algoritmo de Rango-Doppler es un algoritmo de enfoque que procesa el espacio de señal para generar la imagen final. El RDA está basado en la aplicación del filtro adaptado en cada dimensión. En primer lugar, se aplica el filtro adaptado a la señal en la dirección de rango, concentrando la energía de la señal en la celda de rango correspondiente a su posición real en el rango inclinado. A este proceso se lo denomina compresión en rango. Posteriormente, tiene lugar el procedimiento 


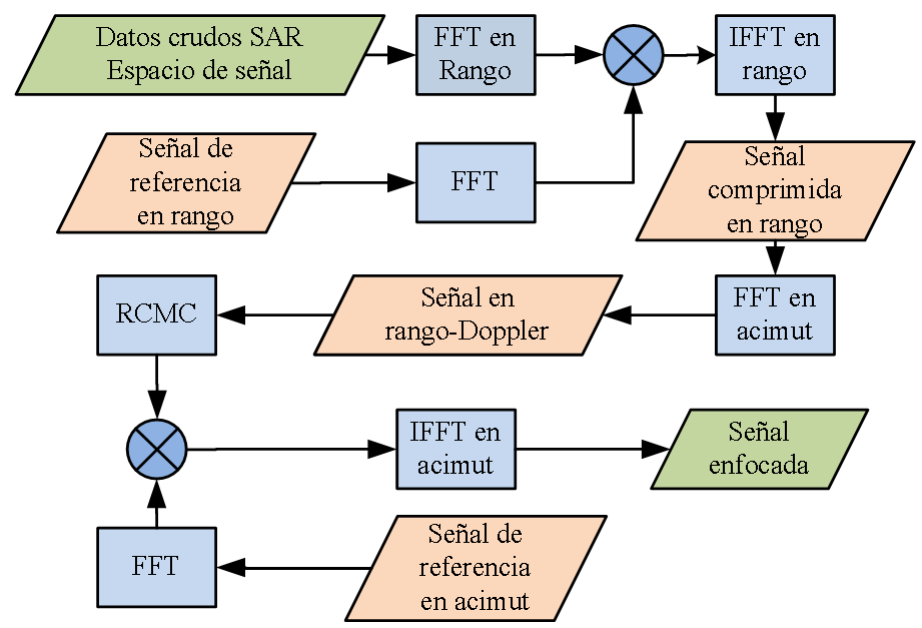

Figura 2.9: Esquema RDA

de corrección de migración de celda de rango (RCMC), y finalmente se realiza la compresión en acimut con la aplicación del filtro adaptado en esa dirección. Una característica distintiva del RDA es que la aplicación de cada filtro adaptado se implementa en el dominio de la frecuencia, mediante la multiplicación de las transformadas de Fourier de las señales involucradas. Adicionalmente, la RCMC se realiza en el dominio rango-Doppler, lo que le da el nombre al algoritmo. La Fig. 2.9 muestra esquemáticamente el procedimiento, donde las siglas FFT e IFFT indican la transformada rápida de Fourier directa e inversa respectivamente.

En primer lugar debe definirse el filtro adaptado en rango $h_{r}(\tau)$ y su transformada de Fourier $H_{r}\left(f_{\tau}\right)=\mathfrak{F}\left\{h_{r}\right\}\left(f_{\tau}\right)$. Recordando la expresión (2.12), $h_{r}$ es una chirp de duración $T_{r}$, de manera que $H_{r}$ resulta:

$$
H_{r}\left(f_{\tau}\right)=\operatorname{rect}\left(\frac{f_{\tau}}{K_{r} T_{r}}\right) \exp \left(-j \pi \frac{f_{\tau}^{2}}{K_{r} T_{r}}\right) .
$$

A cada fila del espacio de señal de señal SAR $s_{0}(\tau, \eta)$, se le aplica la transformada de Fourier para obtener su espectro en dicha dirección, $S_{0}\left(f_{\tau}, \eta\right)$. Cada fila se multiplica por $H_{r}$ para realizar la compresión en rango, y luego se antitransforma para obtener la señal nuevamente en el dominio $(\tau, \eta)$. Obviando los detalles del cálculo y utilizando la aproximación parabólica de $R(\eta)$ dentro de la exponencial, 
la señal en este punto resulta

$$
\begin{aligned}
s_{r c}(\tau, \eta) & =\mathfrak{F}^{-1}\left\{S_{0}\left(f_{\tau}, \eta\right) H_{r}\left(f_{\tau}\right)\right\} \\
& =A_{o b j} p_{r}\left(\tau-\frac{2 R(\eta)}{c}\right) w_{a}(\eta) \exp \left\{-j 4 \pi f_{0} \frac{R_{0}}{c}\right\} \exp \left\{-j \pi K_{a} \eta^{2}\right\},
\end{aligned}
$$

donde $p_{r}$ es la función sinc que resulta de la compresión del pulso en tiempo rápido.

La expresión de $s_{r c}$ evidencia claramente la modulación de frecuencia mediante la dependencia cuadrática de la fase con el tiempo de acimut en la última exponencial (señal chirp en acimut). Además, el retardo $2 R(\eta) / c$ dentro de $p_{r}$ indica la RCM.

La señal $s_{r c}$ es transformada al dominio de Fourier en la dirección de acimut para obtener su expresión en rango-Doppler, $S_{r d}$. Debido a la característica chirp en acimut, la relación entre frecuencia y tiempo es lineal en acimut $f_{\eta}=-K_{a} \eta$ de manera $S_{r d}$ puede obtenerse fácilmente reemplazando $\eta=-f_{\eta} / K_{a}{ }^{3}$ :

$$
S_{r d}\left(\tau, f_{\eta}\right)=A_{o b j} p_{r}\left(\tau-\frac{2 R_{r d}\left(f_{\eta}\right)}{c}\right) w_{a}\left(f_{\eta}\right) \exp \left\{-j 4 \pi f_{0} \frac{R_{0}}{c}\right\} \exp \left\{j \pi \frac{f_{\eta}^{2}}{K_{a}}\right\},
$$

donde $R_{r d}\left(f_{\eta}\right)=R\left(\eta=f_{\eta} / K_{a}\right)=R_{0}+\lambda^{2} R_{0} f_{\eta}^{2} / 8 V_{r}$.

\section{Corrección de la migración en celda de rango}

La cantidad $\Delta R\left(f_{\eta}\right)=\lambda^{2} R_{0} f_{\eta}^{2} / 8 V_{r}$ representa el desplazamiento del objetivo en función de la frecuencia de acimut, para cada $R_{0}$. Es la migración en rango que debe corregirse. Para ello debe computarse $\Delta R\left(f_{\eta}\right)$ para cada $f_{\eta}$ y descontarlo de la trayectoria del objetivo en el dominio rango-Doppler, de manera que la parábola descripta por $R_{r d}$ se transforme en una recta $R_{r d}=R_{0}$. Teniendo en cuenta que cada celda de rango (columna de datos en el espacio rango-Doppler), representa una distancia $\Delta_{\text {rango }}=c / 2 f_{m}$, la corrección gruesa se realiza desplazando a izquierda los datos una cantidad igual a la parte entre del cociente $R_{r d} / \Delta_{\text {rango }}$, para cada $f_{\eta}$

\footnotetext{
${ }^{3}$ Formalmente este reemplazo está justificado al emplearse el Principio de Fase Estacionaria para obtener la transformada de Fourier (Cumming y Wong, 2005, p.74)
} 
(cada línea de datos). La corrección fina, correspondiente a la parte fraccional del cociente, no puede realizarse por desplazamiento y debe realizarse por interpolación.

Luego de haber realizado la RCMC, la dependencia del tiempo en rango con el de acimut es removida del argumento de $p_{r}$ :

$$
S_{r d}\left(\tau, f_{\eta}\right)=A_{o b j} p_{r}\left(\tau-\frac{2 R_{0}}{c}\right) w_{a}\left(f_{\eta}\right) \exp \left\{-j 4 \pi f_{0} \frac{R_{0}}{c}\right\} \exp \left\{j \pi \frac{f_{\eta}^{2}}{K_{a}}\right\} .
$$

\section{Señal Enfocada}

Finalmente, la aplicación de un filtro adaptado a la señal chirp en acimut remueve la dependencia cuadrática de la fase. Su expresión en el dominio transformado es $H_{a c}\left(f_{\eta}\right)=\exp \left\{-j \pi f_{\eta}^{2} / K_{a}\right\}$. La señal en el dominio original resulta:

$$
\begin{aligned}
s_{a c}(\tau, \eta) & =\mathfrak{F}^{-1}\left\{S_{r d}\left(\tau, f_{\eta}\right) H_{a c}\left(f_{\eta}\right)\right\} \\
& =A_{o b j} p_{r}\left(\tau-\frac{2 R_{0}}{c}\right) p_{a}(\eta) \exp \left\{-j 4 \pi f_{0} \frac{R_{0}}{c}\right\},
\end{aligned}
$$

donde $p_{a}$ es una función sinc en acimut que resulta de la remoción de la fase cuadrática en esa dirección.

La expresión (2.23) es el resultado del algoritmo RDA para el enfoque correspondiente a un objetivo puntual. Se observa que el sinc $p_{r}$ está centrado en $\tau=2 R_{0} / c$ que es el tiempo de ida y vuelta hasta la celda de rango donde se ubica el objetivo. Por otro lado, el sinc en acimut $p_{a}$ está centrado en $\eta=0$. Recordando que $\eta$ mide el tiempo lento para el cual el Doppler en acimut se anula, se observa que el objetivo fue registrado en su posición de Doppler nulo en acimut. Por último, el factor exponencial indica el desfasaje introducido por el viaje de ida y vuelta del pulso hasta el objetivo. 


\subsubsection{Resultados para datos simulados}

El procedimiento del apartado anterior se aplicó a los datos simulados previamente para un target puntual, con la implementación basada en Schlutz (2009). La Fig. 2.10 muestra los datos comprimidos en rango, describiendo una parábola que da cuenta del efecto RCM. La Fig. 2.11 muestra el resultado de la RCMC. Se observa que la mayoría de la energía de la señal se concentra ahora en una sola celda de rango (columna de datos).

El resultado de la compresión en acimut se observa en la Fig. 2.12, donde se puede apreciar la que la energía se enfoca en la posición original del objetivo. Se observa también las crestas y los valles a cada lado de dicho punto, tanto en rango como en acimut, debido a la forma sinc de la señal enfocada, dada por la ec. (2.23).

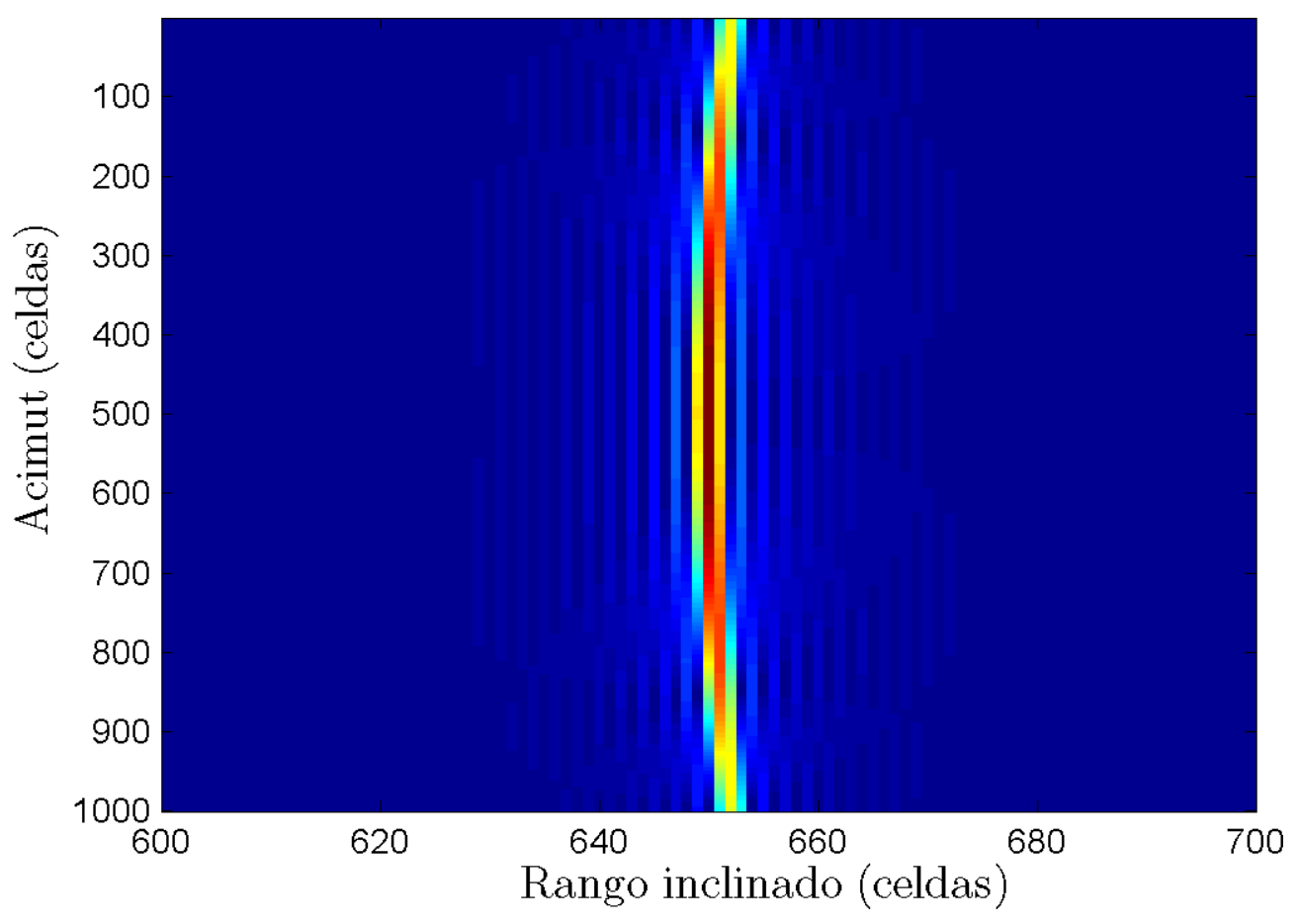

Figura 2.10: Compresión en rango 


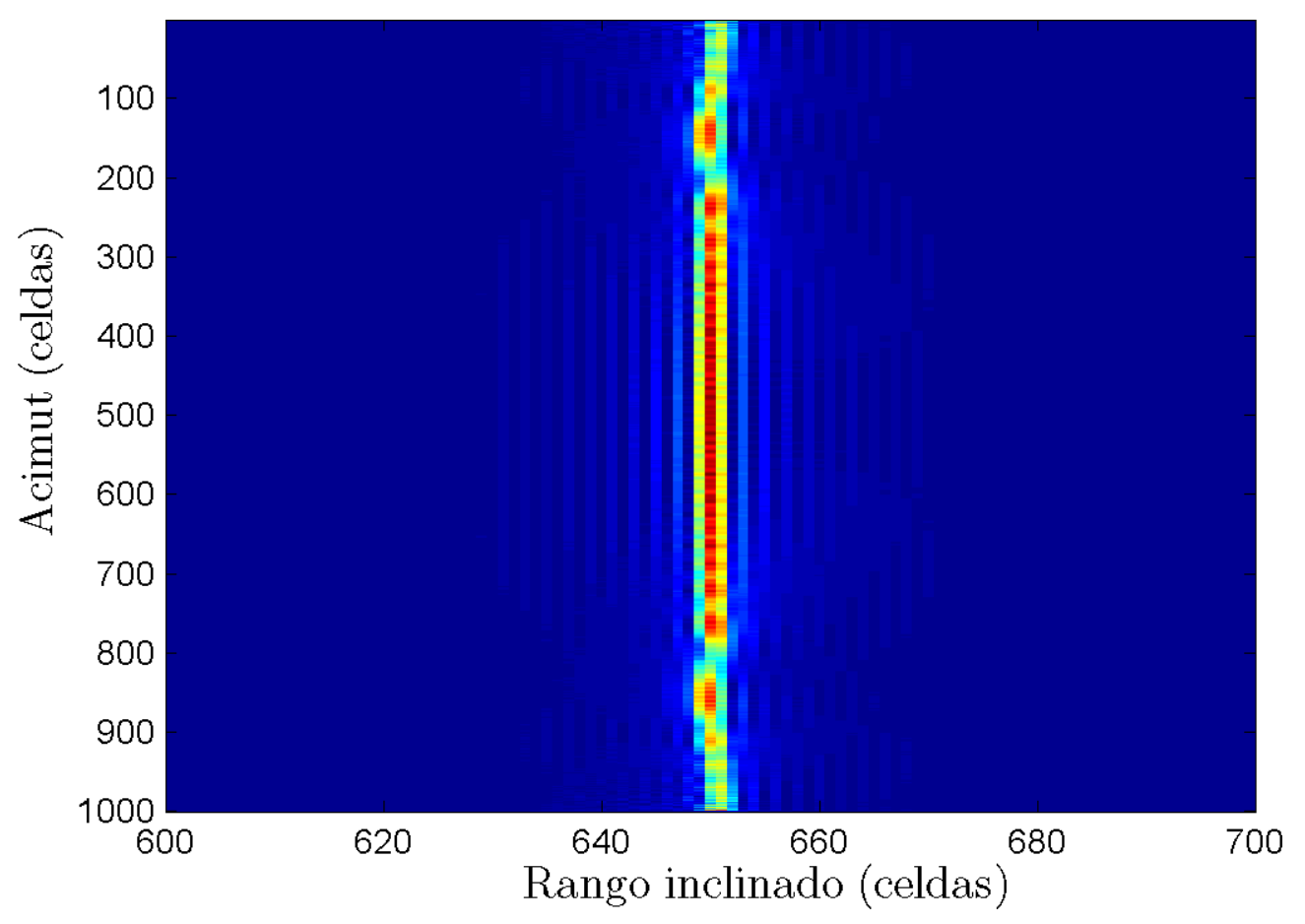

Figura 2.11: Corrección de migración en rango

\subsubsection{Resultados para datos reales}

El algoritmo RDA se aplicó a datos reales de la misión SARAT ${ }^{4}$ con una implementación propia detallada en el apéndice 2.B.

La Fig. 2.13 muestra una imagen de los datos crudos para la polarización hh. La Fig. 2.14 muestra el espacio de señal luego de la compresión en rango y finalmente la Fig. 2.15 muestra la imagen enfocada. El resultado se presenta en rango inclinado sin calibración geométrica. Debe tenerse en cuenta que en el SAR polarimétrico, cada polarización da lugar a una imagen diferente de la misma escena, con sus propias características de amplitud y fase. El estudio del dato enfocado como un vector

\footnotetext{
${ }^{4}$ Imagen SARAT provista por la Comisión Nacional de Actividades Espaciales de Argentina (CONAE) para el proyecto " Métodos y Modelos Estadísticos para Segmentación y Clasificación de Datos SAR (Proyecto n⿳ 19)", llevado a cabo en el marco del "Anuncio de Oportunidad para el Desarrollo de Aplicaciones y Puesta a Punto de Metodologías Utilizando Imágenes SAR banda L Polarimétricas," (C) CONAE(2011).
} 


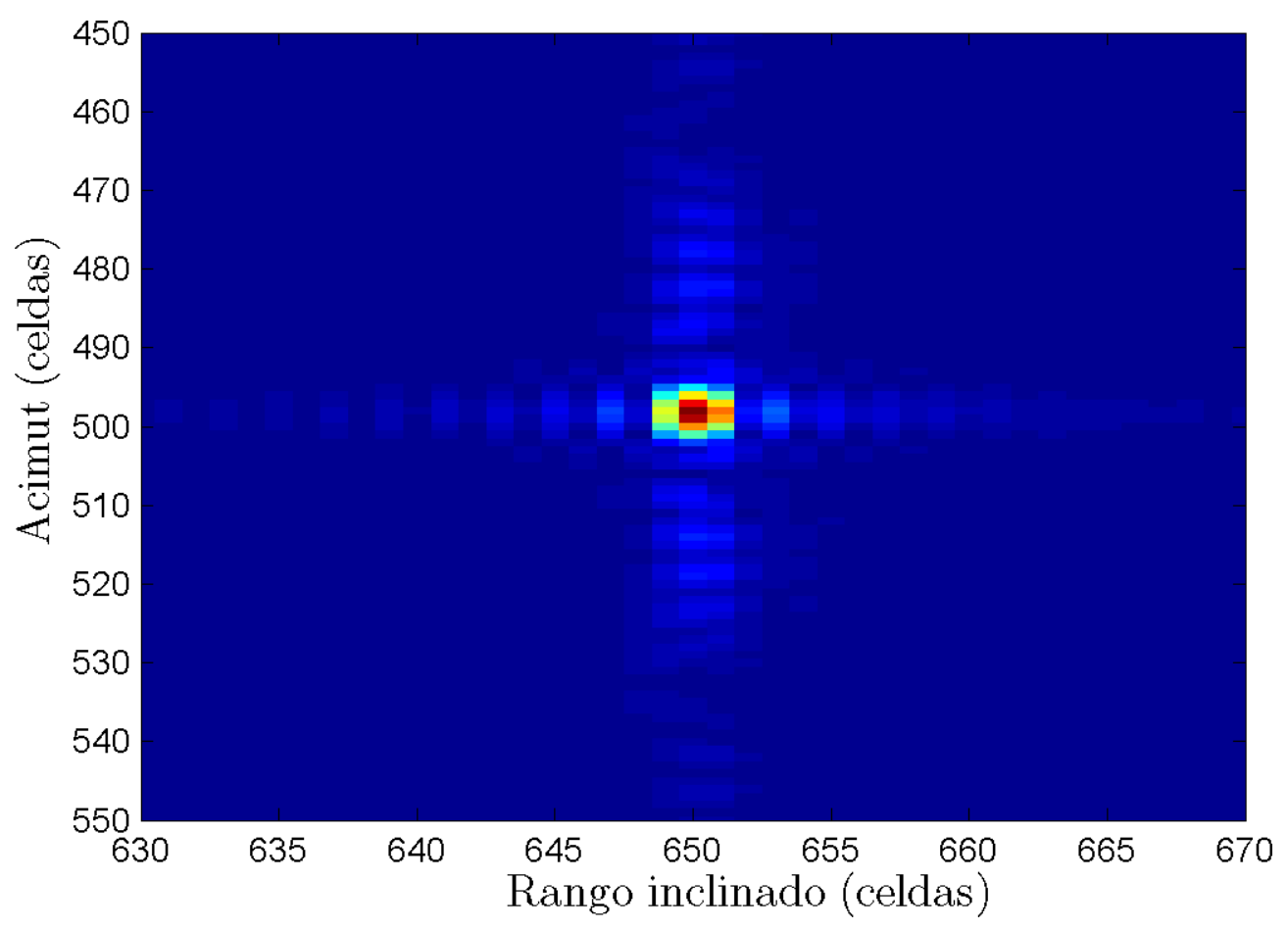

Figura 2.12: Compresión en Acimut

complejo donde cada una de sus componentes proviene de una polarización es lo que permite la extracción de la información útil del terreno para su estudio.

\subsection{Conclusiones}

En este capítulo se analizó el sistema SAR en su modalidad stripmap. A partir de una geometría sencilla y del pulso radar emitido, se derivó la señal recibida y sus características tanto en tiempo lento como en tiempo rápido. Posteriormente se definió el proceso de enfoque como el encargado de transformar el espacio de datos SAR en una imagen útil del terreno. Como ejemplo de algoritmo de enfoque, se estudio el algoritmo en Rango-Doppler y se aplicó tanto a datos sintéticos como a datos SAR reales. En el caso de estos últimos, una implementación propia del RDA permitió el enfoque exitoso de datos de la misión SARAT provistos por CONAE. Los resultados correspondientes fueron presentados en el marco del proyecto "Métodos y Modelos Estadísticos para Segmentación y Clasificación de Datos SAR" en ocasión 


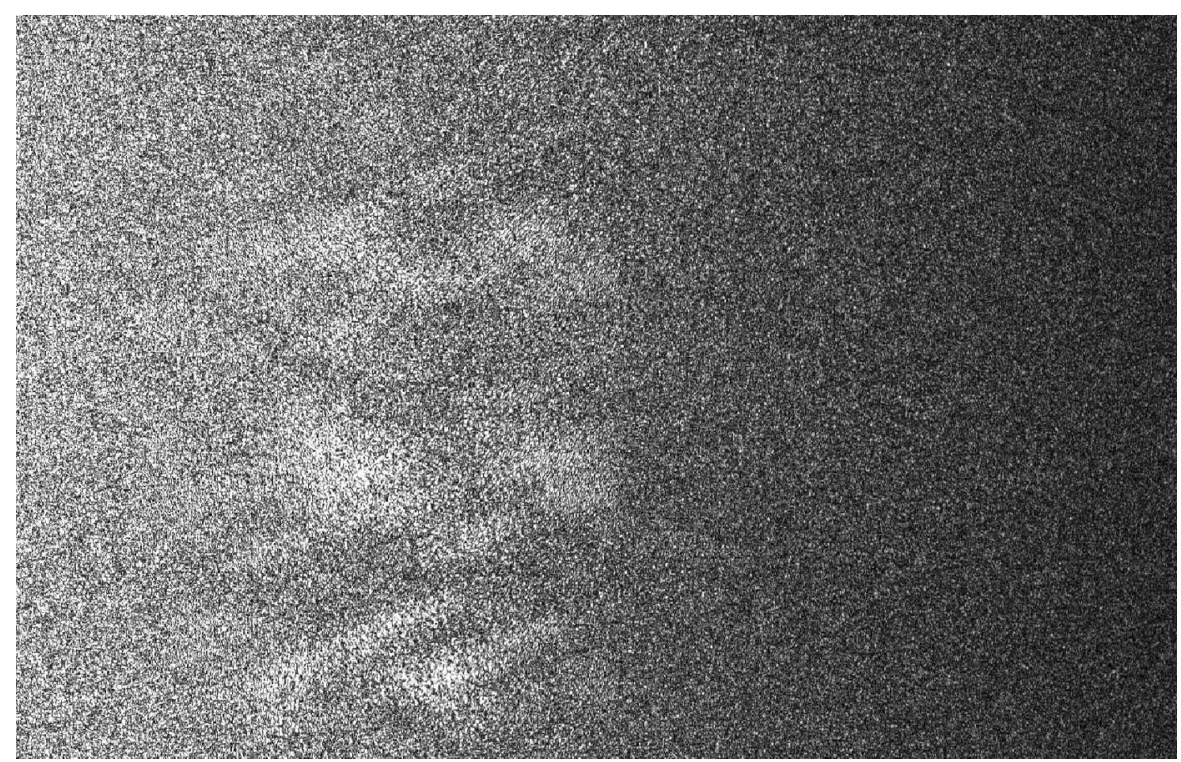

Figura 2.13: Datos SARAT. Espacio de señal (datos crudos).

del "Anuncio de Oportunidad para el Desarrollo de Aplicaciones y Puesta a Punto de Metodologías Utilizando Imágenes SAR banda L Polarimétricas".

Una vez estudiado el proceso de formación de la imagen polarimétrica (datos complejos), se abordará en los siguientes capítulos el estudio de los modelos estadísticos que los describen y en base a ellos, el desarrollo de los algoritmos de clasificación. 


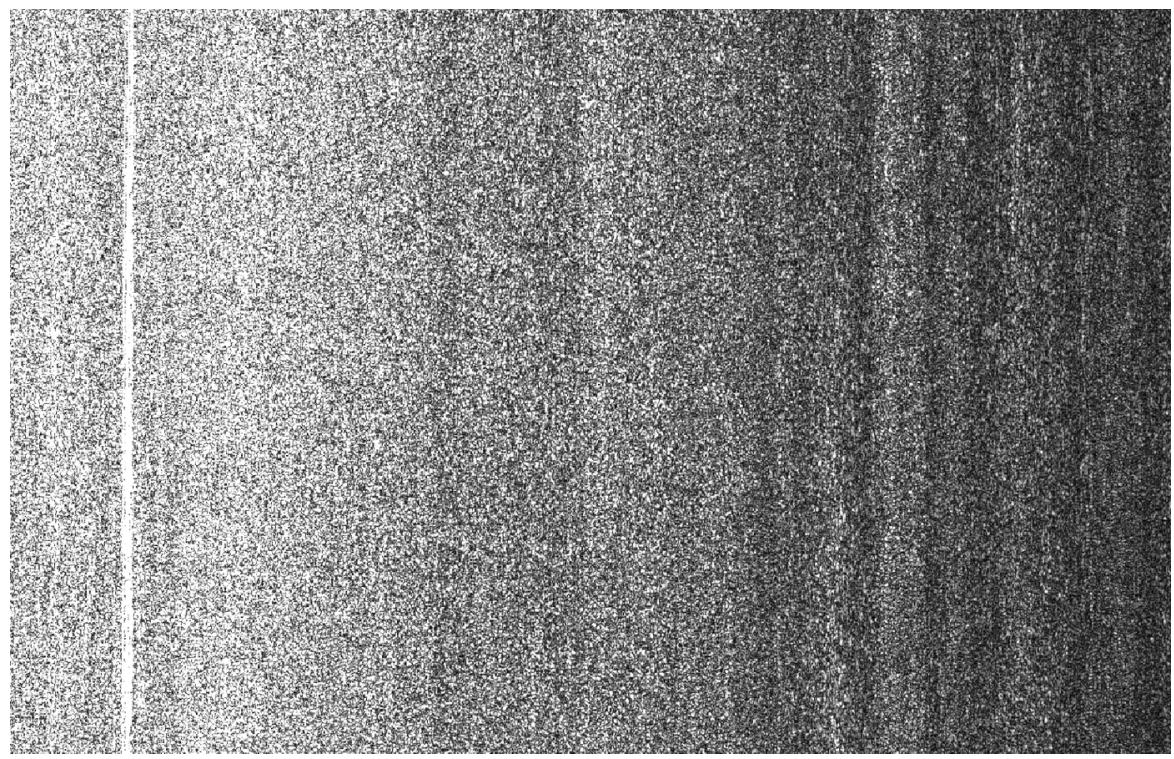

Figura 2.14: Datos SARAT. Compresión en rango.

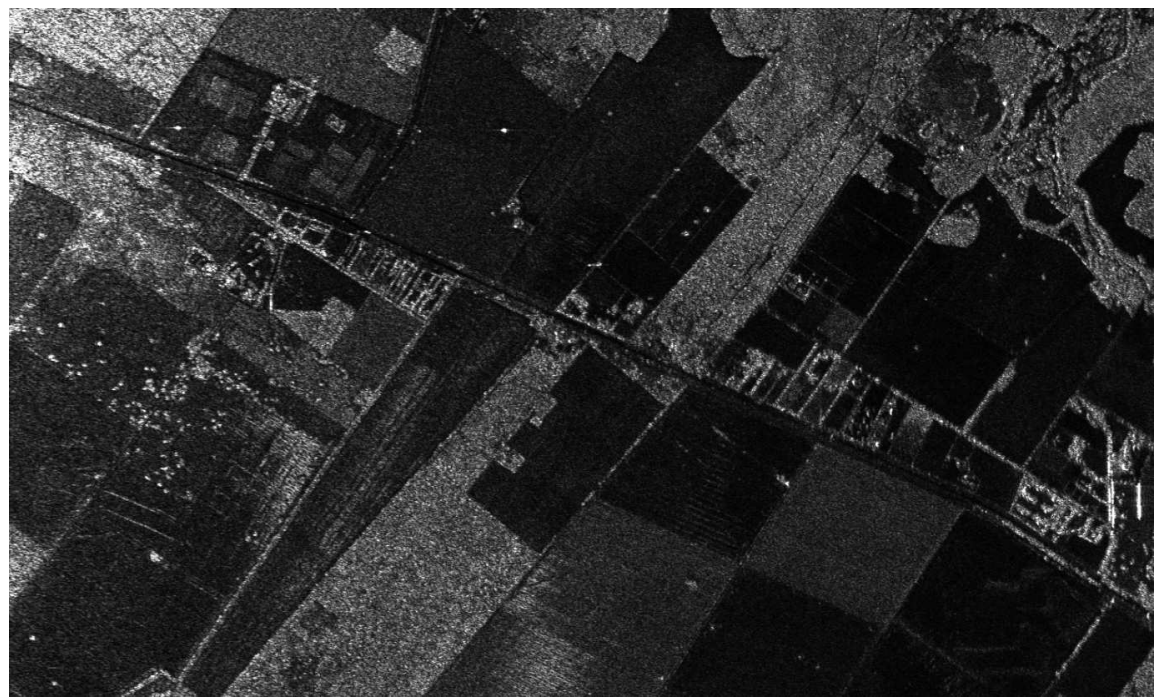

Figura 2.15: Datos SARAT. Compresión en Acimut. 


\section{A. Cálculo de la compresión en rango}

En este apartado se muestran los detalles del cálculo de la compresión de la señal SAR en rango.

Denotando a la señal comprimida en $\operatorname{rango} s_{f}(\tau)$, a la señal recibida $s_{0}^{\prime} \mathrm{y}$ al filtro adaptado $h_{r}(\tau)$ resulta:

$$
\begin{aligned}
s_{f}(\tau)= & \left\{s_{0}^{\prime} * h_{r}\right\}(\tau)=\int_{-\infty}^{\infty} s_{0}^{\prime}(\tau-u) h_{r}(u) d u \\
= & \int_{-\infty}^{\infty} K^{\prime} \operatorname{rect}\left(\frac{\tau-\tau_{0}-u}{T_{r}}\right) \exp \left\{j \pi K_{r}\left(\tau-\tau_{0}-u\right)^{2}\right\} \operatorname{rect}\left(\frac{u}{T_{r}}\right) \\
& \exp \left\{-j \pi K_{r} u^{2}\right\} d u \\
= & K^{\prime} \exp \left\{j \pi K_{r}\left(\tau-\tau_{0}\right)^{2}\right\} \\
& \int_{-\infty}^{\infty} \operatorname{rect}\left(\frac{\tau-\tau_{0}-u}{T_{r}}\right) \operatorname{rect}\left(\frac{u}{T_{r}}\right) \exp \left\{-j 2 \pi K_{r} u\left(\tau-\tau_{0}\right)\right\} d u,
\end{aligned}
$$

Si $\tau_{0}-T_{r} \leq \tau \leq \tau_{0}$, puede escribirse:

$$
\begin{aligned}
s_{f}(\tau) & =K^{\prime} \exp \left\{j \pi K_{r}\left(\tau-\tau_{0}\right)^{2}\right\} \int_{-T_{r} / 2}^{\tau-\tau_{0}+T_{r} / 2} \exp \left\{-j 2 \pi K_{r} u\left(\tau-\tau_{0}\right)\right\} d u \\
& =K^{\prime} \exp \left\{j \pi K_{r}\left(\tau-\tau_{0}\right)^{2}\right\}\left[\frac{\exp \left\{-j 2 \pi K_{r} u\left(\tau-\tau_{0}\right)\right\}}{-j 2 \pi K_{r}\left(\tau-\tau_{0}\right)}\right]_{-T_{r} / 2}^{\tau-\tau_{0}+T_{r} / 2} \\
& =K^{\prime}\left[T_{r}+\tau-\tau_{0}\right] \operatorname{sinc}\left[K_{r}\left(\tau-\tau_{0}\right)\left(T_{r}+\tau-\tau_{0}\right)\right] .
\end{aligned}
$$

Por el contrario, si $\tau_{0} \leq \tau \leq \tau_{0}+T_{r}$ :

$$
\begin{aligned}
s_{f}(\tau) & =K^{\prime} \exp \left\{j \pi K_{r}\left(\tau-\tau_{0}\right)^{2}\right\} \int_{\tau-\tau_{0}-T_{r} / 2}^{T_{r} / 2} \exp \left\{-j 2 \pi K_{r} u\left(\tau-\tau_{0}\right)\right\} d u \\
& =K^{\prime} \exp \left\{j \pi K_{r}\left(\tau-\tau_{0}\right)^{2}\right\}\left[\frac{\exp \left\{-j 2 \pi K_{r} u\left(\tau-\tau_{0}\right)\right\}}{-j 2 \pi K_{r}\left(\tau-\tau_{0}\right)}\right]_{\tau-\tau_{0}-T_{r} / 2}^{T_{r} / 2} \\
& =K^{\prime}\left[T_{r}-\left(\tau-\tau_{0}\right)\right] \operatorname{sinc}\left[K_{r}\left(\tau-\tau_{0}\right)\left(T_{r}-\left(\tau-\tau_{0}\right)\right)\right] .
\end{aligned}
$$


Las ecs. (2.27) y (2.28) pueden reducirse a una única expresión:

$$
\left.s_{f}(\tau)=K^{\prime}\left[T_{r}-\left|\tau-\tau_{0}\right|\right] \operatorname{sinc}\left[K_{r}\left(\tau-\tau_{0}\right)\left(T_{r}-\mid \tau-\tau_{0}\right) \mid\right)\right]
$$

El resultado es una función sinc modulada por una función triangular, ambas centradas en $\tau=\tau_{0}$. Teniendo en cuenta que la variación de esta última es mucho más lenta que la del sinc alrededor de dicho punto y que el sinc se extingue mucho más rápidamente, obtiene finalmente

$$
s_{f}(\tau) \simeq K^{\prime} T_{r} \operatorname{sinc}\left[K_{r} T_{r}\left(\tau-\tau_{0}\right)\right]
$$

que es la expresión de la ec.(2.13). 


\section{B. RDA: Implementación en MATLAB}

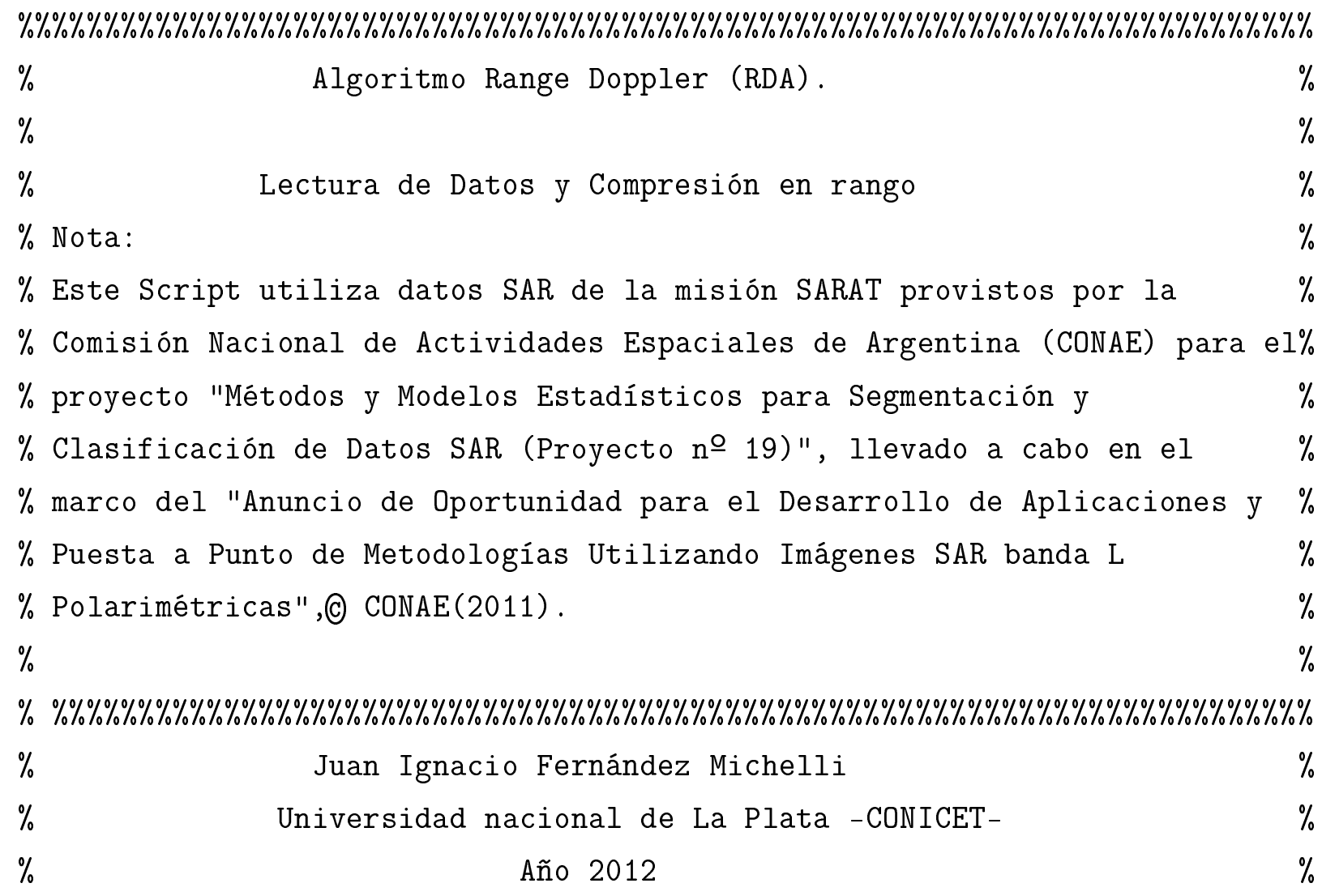

$\% \% \% \% \% \% \% \% \% \% \% \% \% \% \% \% \% \% \% \% \% \% \% \% \% \% \% \% \% \% \% \% \% \% \% \% \% \% \% \% \% \% \% \% \% \% \% \% \% \% \% \% \% \% \% \% \% \% \% \% \% \% \% \% \% \% \% \% \% \%$

clear all;

close all;

tic;

ArchivoDatos='W616T49245327.hh.raw';

ArchivoChirp='W616T49245327.hh. chirp';

\%Parámetros de la plataforma

vel=107.84; $\%$ velocidad de la Plataforma [m/s]

AltPlat $=6075.63 ; \quad \%$ altura de la plataforma [m] 


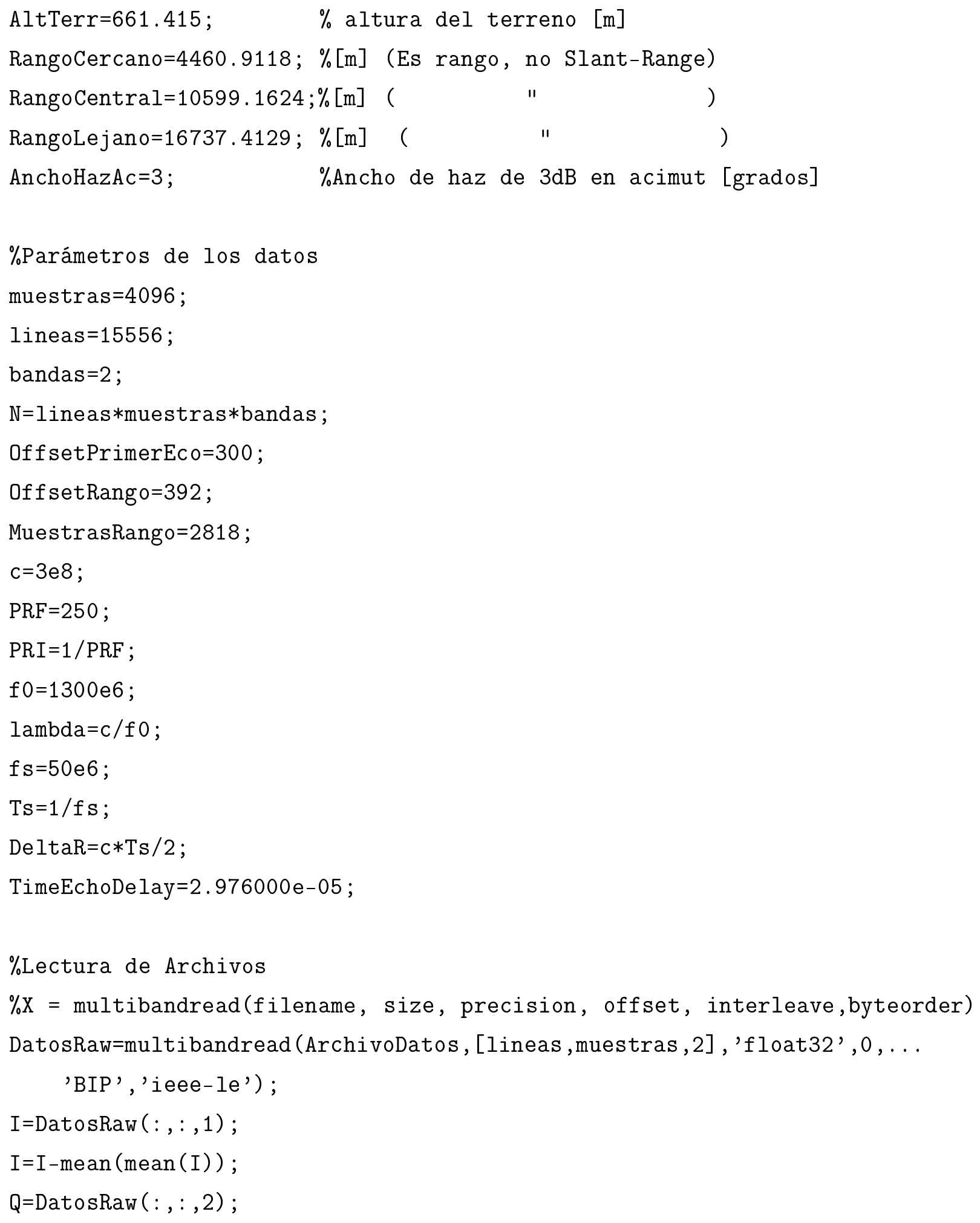


$\mathrm{Q}=\mathrm{Q}-\operatorname{mean}(\operatorname{mean}(\mathrm{Q}))$;

clear DatosRaw;

ChirpData=multibandread (ArchivoChirp, $[1,501,2]$, 'float32', 0, 'BIP', ...

'ieee-le');

$\%$ Carga de datos útiles

DatosComplTotales $=\mathrm{I}+1 \mathrm{i} * \mathrm{Q}$;

clear I Q;

DCompl=DatosComplTotales ; \% (OffsetPrimerEco+1: end, OffsetPrimerEco+1:end);

[n_acim,n_rango]=size (DCompl);

ChirpI=ChirpData $(1,:, 1)$;

ChirpQ=ChirpData $(1,:, 2)$;

ChirpComp=ChirpI+1i*ChirpQ;

l=length (ChirpComp);

clear ChirpI ChirpQ DatosComplTotales;

ChirpComp $=\left[\right.$ ChirpComp, zeros $\left(1, n_{-}\right.$rango-1) $]$; completo con ceros

DCompl= [DCompl, $\operatorname{zeros}\left(n_{-}\right.$acim , 1-1)];

FFT_Chirp=fft (ChirpComp);

EspectroRango=fft (DCompl, []$, 2)$;

imshow (imadjust (abs (DCompl $(870: 1740,1817: 3000)) / a b s(\max (\operatorname{DCompl}(:))), \ldots$

$$
[0.1]),[]) \text {; }
$$

set (gcf, 'units', 'centimeters') ;

set (gcf, 'position', [ $\left.\begin{array}{llll}5 & 5 & 15 & 12\end{array}\right]$ );

savefig('crudos', gcf, 'tiff');

\%Compresión en Rango

\%Convolución del pulso chirp con los datos en rango, mediante transformadas FFT_Chirp_matriz=repmat(FFT_Chirp,n_acim,1); \%Formo una matriz para 
\% multiplicar más fácil

FFT_Compr_Rango=conj (FFT_Chirp_matriz).*EspectroRango;\%multiplico

$\%$ fila por fila

Compr_Rango=ifft(FFT_Compr_Rango, [] ,2); \%Recupero la señal en tiempo

Compr_Rango=circshift (Compr_Rango, [0 1-1] );

Compr_Rango=Compr_Rango $\left(:, 1+1: n_{-}\right.$rango $\left.+1-1\right)$;

toc

save ('Datos_enfocados_en_rango.mat', 'Compr_Rango');

\% \% \% \% \% \% \% \% \% \% \% \% \% \% \% \% \% \% \% \% \% \% \% \% \% \% \% \% \% \% \% \% \% \% \% \% \% \% \% \% \% \% \% \% \% \% \% \% \% \% \% \% \% \% \% \% \% \% \% \% \% \% \% \% \% \% \% \% \% \% \% \% \% \% \%

$\% \quad$ Algoritmo Range Doppler (RDA) . \%

$\% \quad \%$

$\% \quad$ Compresión en Acimut $\%$

\% Nota: $\%$

\% Este Script utiliza datos SAR de la misión SARAT provistos por la \%

\% Comisión Nacional de Actividades Espaciales de Argentina (CONAE) para el\%

\% proyecto "Métodos y Modelos Estadísticos para Segmentación y \%

\% Clasificación de Datos SAR (Proyecto nㅇ 19)", llevado a cabo en el \%

\% marco del "Anuncio de Oportunidad para el Desarrollo de Aplicaciones y \%

\% Puesta a Punto de Metodologías Utilizando Imágenes SAR banda L \%

\% Polarimétricas",(C) CONAE(2011). $\%$

$\%$

$\%$ \% \% \% \% \% \% \% \% \% \% \% \% \% \% \% \% \% \% \% \% \% \% \% \% \% \% \% \% \% \% \% \% \% \% \% \% \% \% \% \% \% \% \% \% \% \% \% \% \% \% \% \% \% \% \% \% \% \% \% \% \% \% \% \% \% \% \% \% \% \% \% \% \% \% \% \%

$\% \quad$ Juan Ignacio Fernández Michelli \%

$\% \quad$ Universidad nacional de La Plata -CONICET- \%

$\%$ Año $2012 \%$

\% \% \% \% \% \% \% \% \% \% \% \% \% \% \% \% \% \% \% \% \% \% \% \% \% \% \% \% \% \% \% \% \% \% \% \% \% \% \% \% \% \% \% \% \% \% \% \% \% \% \% \% \% \% \% \% \% \% \% \% \% \% \% \% \% \% \% \% \% \% \% \% \% \% \% \% \% \% \% \% \%

$C=299792458$;

$f c=1.3 e 9$;

$f_{S}=50 e 6$; 


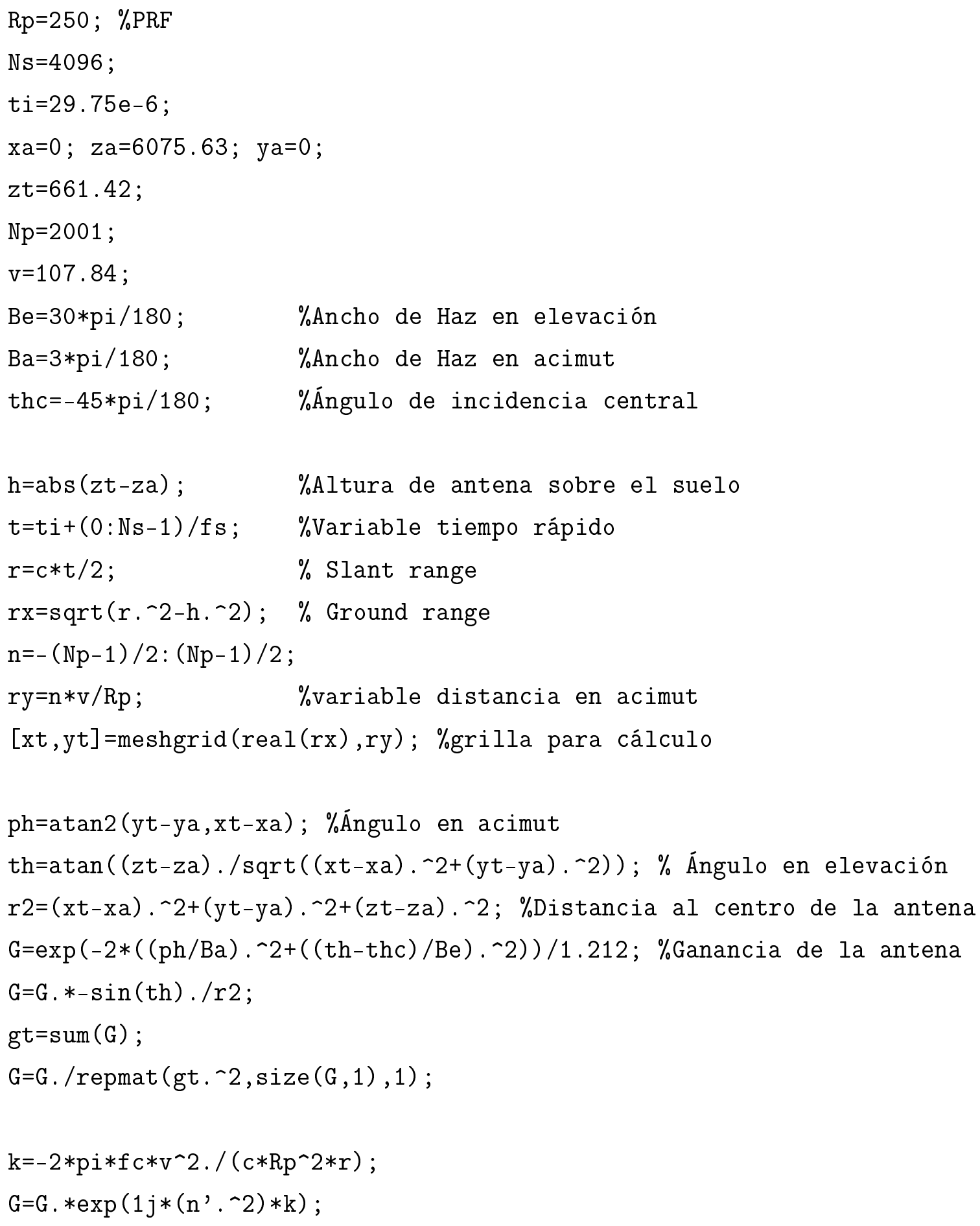


$\mathrm{y}=$ Compr_Rango;

clear Compr_Rango;

$[\mathrm{a}, \mathrm{b}]=\operatorname{size}(\mathrm{y})$;

$\mathrm{G}=\mathrm{G}(:, 1: \mathrm{b})$;

$I=\operatorname{abs}(\operatorname{ifft}(\operatorname{conj}(f f t([0 * y ; G])) . * f f t([y ; 0 * G])))$;

figure;

imshow (imadjust $(\operatorname{abs}(y(870: 1740,1817: 3000)) / \operatorname{abs}(\max (y(:))),[0 \quad 0.005]),[])$;

set (gcf , 'units', 'centimeters') ;

set(gcf, 'position', [ $\left.\begin{array}{llll}5 & 5 & 15 & 12\end{array}\right]$ );

savefig('compr_rango',gcf, 'tiff');

clear y G

$\mathrm{I} 8=\mathrm{I}(1: 8: 17552,:)+\mathrm{I}(2: 8: 17552,:)+\mathrm{I}(3: 8: 17552,:)+\mathrm{I}(4: 8: 17552,:)+\ldots$

$I(5: 8: 17552,:)+\mathrm{I}(6: 8: 17552,:)+\mathrm{I}(7: 8: 17552,:)+\mathrm{I}(8: 8: 17552,:)$;

clear I

$\operatorname{rx}=\operatorname{rx}(:, 1: b)$;

figure;

pcolor (real (rx (580:1736)) , - (870:1740)*v/Rp*8, I8 (870:1740, 580:1736));

shading interp;axis image;

is $=\operatorname{sort}(I 8(:))$;

colormap gray;

$\operatorname{caxis}([0$ is (round $(.9 * \operatorname{numel}(\mathrm{I} 8)))])$;

axis off;

set (gcf, 'units', 'centimeters ') ;

set(gcf, 'position', [ $\left.\begin{array}{llll}5 & 5 & 15 & 12\end{array}\right]$ );

savefig('enfocada',gcf, 'tiff') ; 


\section{Capítulo 3}

\section{Datos Polarimétricos y Modelos Esta- dísticos}

\subsection{Datos SAR Polarimétricos}

Un esquema sencillo para entender la interacción del pulso radar con el objetivo es el descripto en la Fig. 3.1. La onda electromagnética incidente emitida por el pulso radar viaja a través del espacio, incide sobre un objetivo interactuando con él y parte de la energía es re-irradiada como una nueva onda electromagnética. Debido a dicha interacción, la onda irradiada posee características diferentes a la onda incidente. El objetivo de interés es poder caracterizar esta nueva onda, de manera de poder extraer información del objetivo con el cual interactuó.

En el caso general, la expresión fundamental que relaciona la potencia de la onda incidente con la onda refleja es la denominada Ecuación de Radar (Richards, 2005, p.11):

$$
P_{r}=\frac{P_{t} G_{t}}{4 \pi R_{t}^{2}} \sigma \frac{A_{r}}{4 \pi R_{r}^{2}},
$$

donde $P_{r}$ es la potencia recibida. El primer factor está completamente determinado por sistema emisor: $P_{t}$ es la potencia transmitida, $G_{t}$ es la ganancia de la antena transmisora y $R_{t}$ es la distancia entre dicha antena y el objetivo. El último factor depende exclusivamente del sistema receptor: $A_{r}$ es la apertura efectiva de la antena 


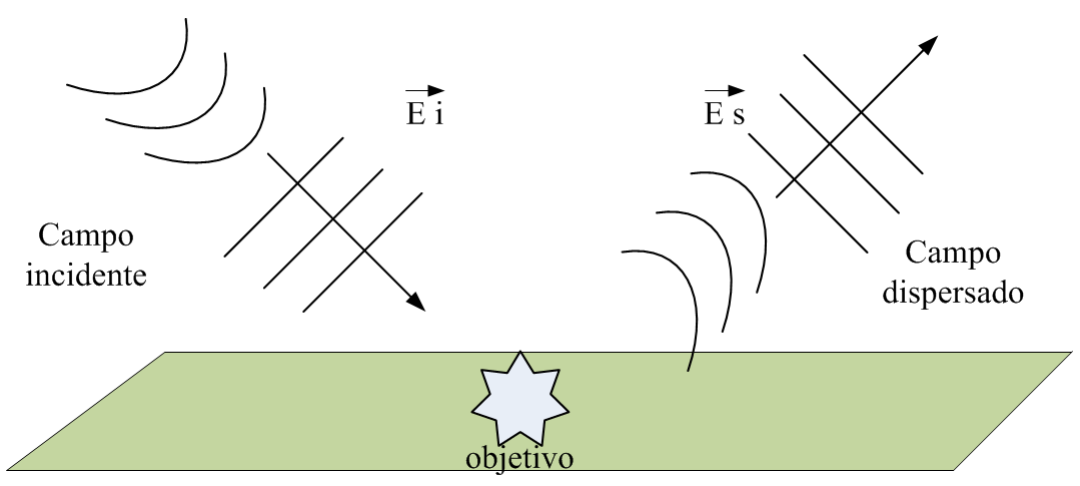

Figura 3.1: Proceso de dispersión.

receptora y $R_{r}$ es la distancia entre ésta y el objetivo. En un radar monoestático, sólo existe una antena, de manera que $R_{r}=R_{t}$. El factor $\sigma$ se denomina sección transversal de Radar y cuantifica cuánta potencia de la que incide en el objetivo es re-irradiada . La sección transversal de radar tiene unidades de superficie. Para independizar la cantidad de potencia que refleja el objetivo del ángulo de la onda incidente, $\sigma$ se define como la sección transversal de un radiador isotrópico equivalente que refleja la misma potencia que el objetivo original en la dirección observada:

$$
\sigma=4 \pi R \frac{\left|\vec{E}_{s}\right|^{2}}{\left|\vec{E}_{i}\right|^{2}}=4 \pi R^{2}|S|^{2} .
$$

$S \in \mathbb{C}$ se denomina coeficiente de dispersión y $\vec{E}_{i}, \vec{E}_{s}$ son los campos eléctricos vectoriales incidente y dispersado respectivamente. La sección transversal de radar depende en general de varios factores, como la frecuencia, ángulo de incidencia, polarización de la onda incidente, y características geométricas y dieléctricas del objetivo.

La Ec. (3.1) aplica para objetivos cuyas dimensiones son más pequeñas que la huella de la antena (objetivos puntuales), de manera que son completamente iluminados por ésta. En el caso SAR, interesan los denominados objetivos extendidos o distribuidos, es decir, aquellos de extensión mayor a la huella de antena. En esta situación, es útil representarlo como un conjunto de objetivos puntuales, cada uno de los cuales contribuye con una porción de la potencia re-irradiada, como se esquematiza en la Fig. 3.2. 


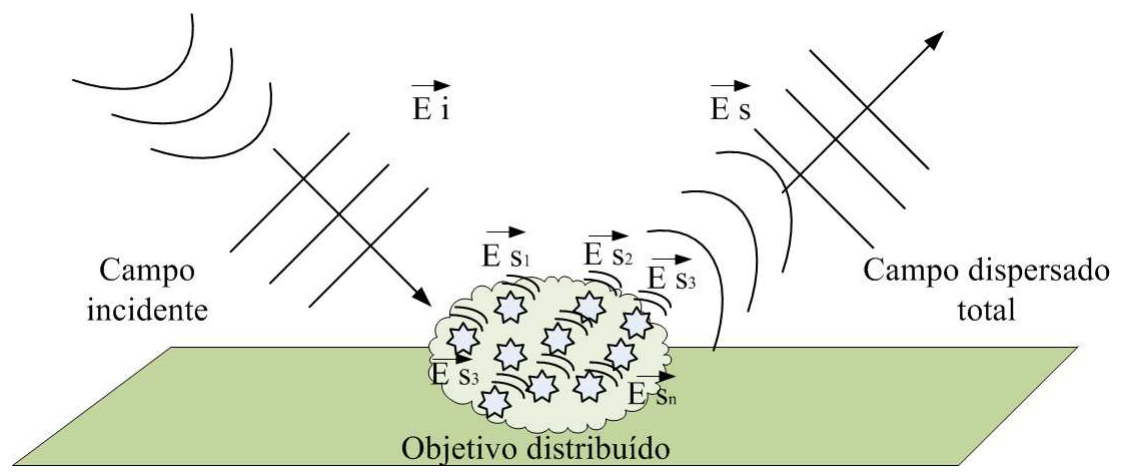

Figura 3.2: Proceso de dispersión en blancos distribuídos.

El campo dispersado $\vec{E}_{s}$ está formado por la suma coherente de los campos dispersados por cada objetivo puntual. La sección transversal se redefine en su versión diferencial $d \sigma=\sigma^{0} d s$, donde $d s$ es el diferencial de superficie y $\sigma^{0}$ es la sección transversal de radar por unidad de superficie. Por lo tanto, la Ec. (3.1) toma la siguiente forma diferencial:

$$
d P_{r}=\frac{P_{t} G_{t}}{4 \pi R_{t}^{2}} \sigma^{0} \frac{A_{r}}{4 \pi R_{r}^{2}} d s
$$

la cual debe integrarse sobre toda el área de la huella de la antena para obtener la potencia total.

Debe notarse que mientras que la Ec. (3.1) describe un problema determinístico para un objetivo puntual, Ec. (3.3) describe un problema estadístico, en el que $\sigma^{0}$ relaciona el promedio de la densidad de potencia dispersada con el promedio de la densidad de potencia incidente:

$$
\sigma^{0}=\frac{4 \pi R^{2}}{\text { Área }} \frac{\left\langle\left|\vec{E}_{s}\right|^{2}\right\rangle}{\left|\vec{E}_{i}\right|^{2}} .
$$

El parámetro $\sigma^{0}$ es adimensional y se utiliza para caracterizar el área iluminada por el radar. Análogamente a la sección transversal de radar, conserva su dependencia con la frecuencia, ángulo de incidencia y polarización. Esta última dependencia se aprovecha para la caracterización polarimétrica del objetivo, considerando que si la onda incidente posee polarización $p$ y se recibe con una antena de polarización $q$, 
entonces

$$
\sigma_{q p}^{0}=\frac{4 \pi R^{2}}{\text { Área }} \frac{\left\langle\left|\vec{E}_{q}\right|^{2}\right\rangle}{\left|\vec{E}_{p}\right|^{2}}=4 \pi\left|s_{q p}\right|^{2}
$$

es una medida de la respuesta del objetivo en la polarización $q$ cuando se lo incide con polarización $p$, es decir, una sección de radar normalizada dependiente de la polarización.

\subsubsection{Matriz de dispersión $S$}

El parámetro $\sigma_{q p}^{0}$ proporciona información de la potencia reflejada para cada polarización. Sin embargo, para extraer información de fase de la misma, es necesario aprovechar la naturaleza vectorial de las ondas polarizadas. Para ello los vectores de Jones del campo eléctrico dispersado se relacionan con el campo incidente con la siguiente expresión (Lee y Pottier, 2009, pp.37,56):

$$
\vec{E}_{s}=\frac{\exp \{-j k r\}}{r} S \vec{E}_{i}=\frac{\exp \{-j k r\}}{r}\left[\begin{array}{ll}
s_{11} & s_{12} \\
s_{21} & s_{22}
\end{array}\right] \vec{E}_{i}
$$

donde $k$ es el módulo del vector de propagación y $r$ es la distancia al objetivo. $S$ es la denominada matriz de dispersión y sus elementos son los coeficientes de dispersión complejos. Cada $s_{i j}$ indica la relación vectorial entre el campo incidente de polarización $j$ y el dispersado con polarización $i$, mientras que el factor $\exp \{-j k r\} / r$ tiene en cuenta la atenuación y el desfasaje por propagación.

En el caso del radar monoestático, $s_{i j}=s_{j i}$, de manera que la matriz $S$ es simétrica.

\subsubsection{Vector objetivo}

Una forma alternativa de representar la matriz $S$ es organizar sus elementos en un vector $\boldsymbol{k}$ denominado vector de objetivo. Formalmente, la vectorización se hace a través del producto interno Hermítico de $S$ con una base ortogonal $\Psi$ :

$$
\boldsymbol{k}=\frac{1}{2} \operatorname{tr}(S \Psi)
$$


donde $\operatorname{tr}(\cdot)$ indica la traza. Por lo tanto, la expresión del vector objetivo depende de la base elegida. Las más comúnmente utilizadas son las bases de Pauli $\Psi_{P}$ y la Lexicográfica $\Psi_{L}$, cuyas expresiones para el caso monoestático son (Lee y Pottier, 2009):

$$
\begin{aligned}
& \Psi_{P}=\sqrt{2}\left\{\left[\begin{array}{ll}
1 & 0 \\
0 & 1
\end{array}\right],\left[\begin{array}{cc}
1 & 0 \\
0 & -1
\end{array}\right],\left[\begin{array}{ll}
0 & 1 \\
1 & 0
\end{array}\right]\right\}, \\
& \Psi_{L}=2\left\{\left[\begin{array}{ll}
1 & 0 \\
0 & 0
\end{array}\right], \sqrt{2}\left[\begin{array}{ll}
0 & 1 \\
0 & 0
\end{array}\right],\left[\begin{array}{ll}
0 & 0 \\
0 & 1
\end{array}\right]\right\} .
\end{aligned}
$$

Cada base da lugar a su correspondiente vector de objetivo $\boldsymbol{k}_{L}$ y $\boldsymbol{k}_{P}$. En este trabajo se utilizará la base $\Psi_{L}$ de manera que se prescindirá del subíndice: $\boldsymbol{k}=$ $\left[s_{11} \sqrt{2} s_{12} s_{22}\right]$.

El vector objetivo, o equivalentemente la matriz de dispersión, representan los datos SAR de más bajo nivel con los que puede construirse una imagen del terreno.

\subsubsection{Matrices de Coherencia y Covarianza}

La matriz de dispersión caracteriza completamente a los objetivos estacionarios, es decir, a aquellos cuyas características permanecen fijas y estables durante el tiempo de observación. Sin embargo, dentro de la escena captada por el SAR los objetivos extendidos presentan un comportamiento dinámico en tiempo y espacio que impide su completa caracterización con la matriz $S$ únicamente. Suponiendo que dicho comportamiento corresponde a un proceso estocástico subyacente, una forma más adecuada de describir los datos es mediante su momento de segundo orden con la denominadas matrices de coherencia $T=\mathcal{E}\left\{\boldsymbol{k}_{P} \boldsymbol{k}_{P}^{H}\right\}$ y covarianza $C=\mathcal{E}\left\{\boldsymbol{k}_{L} \boldsymbol{k}_{L}^{H}\right\}$, donde $\mathcal{E}\{\cdot\}$ indica esperanza matemática y $H$ transposición conjugada. Ambas matrices proporcionan la misma información y puede pasarse de una a otra con un simple cambio de base. En la práctica, al trabajar con datos medidos, se calculan las matrices a partir de su estimador muestral remplazando el promedio estadístico por el promedio muestral $T=\left\langle\boldsymbol{k}_{P} \boldsymbol{k}_{P}^{H}\right\rangle, C=\left\langle\boldsymbol{k}_{L} \boldsymbol{k}_{L}^{H}\right\rangle$. A propósitos de este trabajo, 
será de interés la matriz de $C$, cuya expresión es:

$$
C=\left[\begin{array}{ccc}
\left\langle\left|s_{11}\right|^{2}\right\rangle & \sqrt{2}\left\langle s_{11} s_{12}^{*}\right\rangle & \left\langle s_{11} s_{22}^{*}\right\rangle \\
\sqrt{2}\left\langle s_{12} s_{11}^{*}\right\rangle & 2\left\langle\left|s_{12}\right|^{2}\right\rangle & \sqrt{2}\left\langle s_{12} s_{22}^{*}\right\rangle \\
\left\langle s_{22} s_{11}^{*}\right\rangle & \sqrt{2}\left\langle s_{22} s_{12}^{*}\right\rangle & \left\langle\left|s_{22}\right|^{2}\right\rangle
\end{array}\right]
$$

Debido a que SAR trata con objetivos extendidos, las matrices $C$ y $T$ constituyen los datos de menor complejidad capaces de capturar las características polarimétricas del terreno y debido a ello son la base del procesamiento para la gran mayoría de los algoritmos de clasificación y segmentación de imágenes SAR.

\subsubsection{Speckle}

El speckle es un fenómeno que caracteriza a las imágenes obtenidas por iluminación coherente, y que produce en ellas el efecto de moteado con diferentes intensidades. Dentro de la celda de resolución del radar, existen muchos elementos dispersores de dimensiones comparables a la longitud de onda, que interactúan con la señal incidente produciendo reflexiones, como se esquematiza en la Fig. 3.3. Dado que la onda incidente es coherente, cada una de estas reflexiones interactúa entre sí interfiriendo constructiva o destructivamente. Por lo tanto, el nivel de intensidad que se observa para cada píxel, es el resultado de dicha interacción. Dado que el resultado de la interferencia entre ondas depende de su fase, y ésta, a su vez, de la diferencia de camino recorrido por ellas, el fenómeno es dependiente del ángulo incidente. Por lo tanto, además de la longitud de onda, el fenómeno de speckle dependerá del ángulo de incidencia y de la distancia al objetivo.

Denotando con $x_{i}$ e $y_{i}$ a la parte real e imaginaria del fasor de campo eléctrico producido por el $i$-ésimo dispersor, el campo eléctrico resultante será la suma de la contribuciones de los $N_{d}$ dispersores dentro de la celda de resolución ${ }^{1}$ :

$$
E_{T}=x+j y=\sum_{i=1}^{N_{d}}\left(x_{i}+j y_{i}\right) .
$$

\footnotetext{
${ }^{1}$ En la notación fasorial del campo eléctrico se supone una variación armónica temporal que se omite en la expresión.
} 


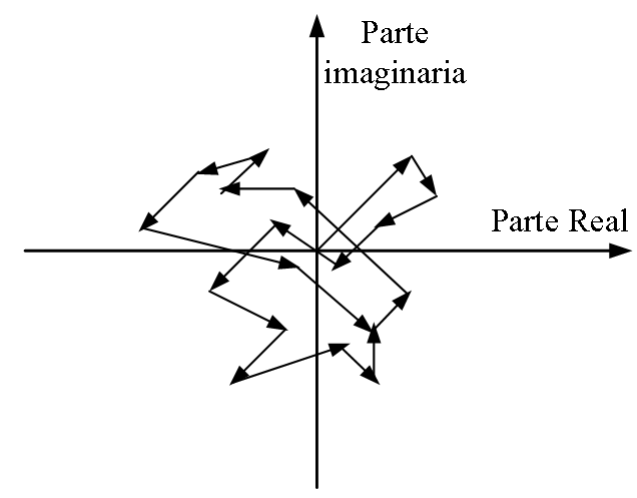

Figura 3.3: Sumatoria de reflexiones.

Este efecto del speckle sobre el campo recibido, junto con las características del terreno determinan la caracterización estadística de los datos.

\subsection{Modelo para datos homogéneos}

Bajo la suposición que en la celda de resolución SAR existe un gran número de elementos dispersores de dimensiones mucho más pequeñas que la longitud de onda, puede suponerse que la fase de las reflexiones $\left(x_{i}+j y_{i}\right)$ está uniformemente distribuida en el intervalo $[-\pi, \pi]$, es decir, el ángulo entre parte real e imaginaria puede tomar cualquier valor con igual probabilidad. Esto se denomina speckle completamente desarrollado. Además, si el efecto de ninguno de los dispersores predomina sobre el resto (terreno homogéneo), por el Teorema del Límite Central, la suma de las componentes reales e imaginarias independientes puede modelarse como una variable aleatoria de fdp gaussiana compleja de media nula y varianza igual a la suma de las varianzas de cada componente.

Esta característica se extiende al vector objetivo $\boldsymbol{k}$, de forma que puede modelarse como una variable aleatoria gaussiana multivariada $\mathcal{N}_{c}^{d}(0, \Sigma)$ cuya fdp es

$$
f_{\boldsymbol{k} ; \Sigma}(\boldsymbol{k} ; \Sigma)=\frac{\exp \left(-\boldsymbol{k}^{H} \Sigma^{-1} \boldsymbol{k}\right)}{\pi^{d}|\Sigma|}
$$


donde $\Sigma=\mathcal{E}\left\{\boldsymbol{k} \boldsymbol{k}^{H}\right\}$ es la matriz de covarianza con simetría hermítica y $d=3$ es la dimensión de $\boldsymbol{k}$. Este modelo describe correctamente los datos provenientes de terrenos homogéneos. En el Cap. 4, se utilizará el modelo gaussiano ${ }^{2}$ para el desarrollo de un clasificador no supervisado.

Además de modelar el vector objetivo, interesa describir estadísticamente a la matriz de covarianza $C$. Si el vector $\boldsymbol{k}$ sigue el modelo (3.11), entonces la matriz $C$ es un estimador de $\Sigma$. Si se promedian $n$ vectores $\boldsymbol{k}$ para calcular $C$,

$$
C=\frac{1}{n} \sum_{i=1}^{n} \boldsymbol{k}_{i} \boldsymbol{k}_{i}^{H},
$$

entonces la variable $Z=n C$ tiene fdp Wishart compleja:

$$
f_{Z ; n, \Sigma}(Z ; n, \Sigma)=\frac{n^{d n}|Z|^{n-d}}{h(n, d)|\Sigma|^{n}} \exp \left[-n \operatorname{tr}\left(\Sigma^{-1} Z\right)\right]
$$

con $h=\pi^{\frac{1}{2} d(d-1)} \prod_{j=0}^{d-1} \Gamma(n-j)$. La fdp Wishart es extensamente utilizada en el procesamiento de datos SAR y constituye la base para modelos de datos polarimétricos más complejos.

\subsubsection{Número equivalente de observaciones}

El fenómeno de speckle degrada la visualización de las imágenes, produciendo un efecto de moteado cuando se observa la imagen de intensidad o amplitud del valor complejo de cada dato. Una medida de esta degradación es la relación señal a ruido SNR de la imagen, definida como el cociente entre la media al cuadrado y la varianza de la intensidad. En base a la expresión (3.10), la intensidad de cada dato complejo para una dada polarización es $I=x^{2}+y^{2}$. Si los datos siguen el modelo gaussiano complejo $\mathcal{N}_{c}\left(0, \sigma^{2}\right)$ y $x$ e $y$ son independientes, entonces $I$ tiene fdp exponencial $\operatorname{Exp}(\sigma)$. Entonces, $\mathcal{E}(I)=\sigma^{2}$ y $\operatorname{Var}(I)=\sigma^{4}$, por lo que $\mathrm{SNR}=1$. Este valor es característico del speckle completamente desarrollado.

\footnotetext{
${ }^{2}$ Se utiliza en realidad un modelo gaussiano menos restrictivo en el que se permite a los datos poseer media.
} 
Para mejorar la SNR, suponiendo que los datos son independientes, se promedian en intensidad de manera de reducir su varianza. Este procedimiento se denomina procesamiento de múltiples observaciones (multilook). Al promediar $n$ datos se obtiene $I_{n}=\sum_{j=1}^{n}\left(x_{j}^{2}+y_{j}^{2}\right) / n$, de manera que $n I_{n}$ tiene fdp Chi-cuadrado con $2 n$ grados de libertad, $\chi^{2}(2 n)$. Recalculando la SNR resulta

$$
\operatorname{SNR}\left(I_{n}\right)=\frac{\mathcal{E}\left(I_{n}\right)^{2}}{\operatorname{Var}\left(I_{n}\right)}=\frac{\left(\sigma^{2}\right)^{2}}{\sigma^{4} / n}=n .
$$

Se observa que al promediar $n$ muestras independientes, se ha mejorado la SNR en un factor $n$, a costa de perder resolución. Debido a este comportamiento, la relación entre el cuadrado de la media y la varianza de los datos de intensidad es un factor de mérito que indica el grado en que una imagen está afectada por speckle. En la práctica, dado que puede existir correlación entre los datos promediados la mejora en SNR resulta menor a $n$, sin embargo, la relación mencionada sigue siendo válida y determina en denominado número equivalente de observaciones (ENL, por sus siglas en inglés).

EL ENL se extiende a datos polarimétricos, y pasa a ser uno de los parámetros de las fdp's que los describen (Anfinsen et al., 2009). Por ejemplo, el caso Wishart de la Ec. (3.13), el parámetro $n$ es el ENL de los datos.

\subsection{Modelo para datos no-homogéneos: la distribu- cion $\mathcal{G}_{p}^{0}$}

Cuando el terreno iluminado por el SAR no presenta las características de homogeneidad descriptas en la Sec. 3.2, los datos observados dejan de seguir la estadística gaussiana y debe considerarse un modelo más complejo. El enfoque más utilizado para describir este tipo de datos es el modelo multiplicativo, donde cada observación es el producto de dos variables aleatorias independientes: una tiene en cuenta el fenómeno speckle y la otra modela la información del terreno. En este marco, en Frery et al. (1997) los autores desarrollaron la familia de distribuciones $\mathcal{G}$ para datos de amplitud, intensidad y complejos de una sola polarización, capaz de describir datos 
con diferentes grados de heterogeneidad. Posteriormente, en Freitas et al. (2005) se extiende el modelo al caso polarimétrico con la distribución $\mathcal{G}_{p}$.

Un caso especial de la familia, la distribución $\mathcal{G}_{p}^{0}$, es capaz de describir datos homogéneos, heterogéneos y muy heterogéneos. Esta distribución será utilizada en el Cap. 5 en el desarrollo de un algoritmo de clasificación no supervisado. A continuación se explicarán sus características.

Partiendo del modelo multiplicativo, cada dato SAR polarimétrico $Z \in \mathbb{C}^{d \times d}$ puede expresarse como el producto $Z=x Y$, donde $x$ es independiente de $Y$. La matriz $Y \in \mathbb{C}^{d \times d}$ contiene el efecto del fenómeno speckle e información sobre las características polarimétricas del terreno, mientras que la variable $x \in \mathbb{R}^{+}$da cuenta de las fluctuaciones alrededor del comportamiento medio. El modelo $\mathcal{G}_{p}^{0}$ surge de asignar una fdp Gamma Inversa a $x, \Gamma^{-1}(-\alpha, \gamma)$, y una fdp Wishart a $Y, \mathcal{W C}(C, n)$ :

$$
\begin{aligned}
f_{x ; \alpha, \gamma}(x ; \alpha, \gamma) & =\frac{\gamma^{-\alpha} x^{\alpha-1}}{\Gamma(-\alpha)} \exp \left(-\frac{\gamma}{x}\right) \\
f_{Y ; n, \Sigma}(Y ; n, \Sigma) & =\frac{n^{n d}|Y|^{n-d}}{h(n, d)|\Sigma|^{n}} \exp \left(-n \operatorname{tr}\left(\Sigma^{-1} Y\right)\right)
\end{aligned}
$$

El parámetro de forma $\alpha<-1$ está directamente relacionado con la textura del terreno, siendo más rugoso cuanto más cercano es a -1 . El parámetro $\gamma$ es el parámetro de escala, de tal forma que si $x \sim \Gamma^{-1}(\alpha, \gamma)$ entonces $a x \sim \Gamma^{-1}(\alpha, a \gamma)$. La fdp $\mathcal{G}_{p}^{0}$ de la variable $Z=x Y$ tiene entonces la siguiente expresión:

$$
f_{Z ; \alpha, \gamma, \Sigma}(Z ; \alpha, \gamma, \Sigma)=\frac{n^{n d} \gamma^{-\alpha} \Gamma(n d-\alpha)}{h(n, d) \Gamma(-\alpha)|\Sigma|^{n}}|Z|^{n-d}\left(n \operatorname{tr}\left(\Sigma^{-1} Z\right)+\gamma\right)^{\alpha-n d}
$$

Los detalles de la derivación de la Ec. (3.17) figuran en el Apéndice 3.A.

Se puede observar que la capacidad de describir terrenos heterogéneos trae como consecuencia una mayor cantidad de parámetros respecto del caso homogéneo. Sin embargo, a diferencia de otras distribuciones basadas en el modelo multiplicativo como la $\mathcal{K}$ (Lee et al., 1994) o la $\mathcal{U}$ (Doulgeris et al., 2012), no depende de funciones complicadas (Bessel y Kummer-U, respectivamente), lo que facilita la estimación de sus parámetros. 
Calculando la media de $Z$ se obtiene $\mathcal{E}\{Z\}=\mathcal{E}\{x\} \mathcal{E}\{Y\}=\mathcal{E}\{x\} \Sigma$. Dado que el modelo multiplicativo establece que $\mathcal{E}\{Z\}=\Sigma$, la media de $x$ debe ser unitaria. Teniendo en cuenta la fdp de la Ec. (3.15), $\mathcal{E}\{x\}=\gamma /(-\alpha-1)$, por lo que es posible expresar $\gamma$ en función de $\alpha$ y obtener una expresión de $\mathcal{G}_{p}^{0}$ independiente de $\gamma$ :

$$
f_{Z ; \alpha, \Sigma}(Z ; \alpha, \Sigma)=\frac{n^{n d}(-\alpha-1)^{-\alpha} \Gamma(n d-\alpha)}{h(n, d) \Gamma(-\alpha)|\Sigma|^{n}}|Z|^{n-d}\left(n \operatorname{tr}\left(\Sigma^{-1} Z\right)+(-\alpha-1)\right)^{\alpha-n d} .
$$

Sin embargo, en este trabajo se conservarán ambos parámetros $\alpha$ y $\gamma$ en la expresión de $\mathcal{G}_{p}^{0}$, sin pérdida de generalidad, para facilitar la implementación del algoritmo descripto en el Cap. 5. 


\section{A. Derivación de la fdp $\mathcal{G}_{p}^{0}$}

Partiendo de la expresión de $Z=x Y$, puede expresarse la fdp de $Z$ de la siguiente manera:

$$
f_{Z}(Z)=\int_{-\infty}^{\infty} f_{Y / x}\left(\frac{Z}{x}\right) f_{x}(x) x^{-2 d} d x
$$

donde se omitió indicar explícitamente la dependencia con los parámetros por simplicidad. La función $f_{Y / x}(\cdot)$ indica la fdp condicional de $Y$ condicionada a $x$ y $x^{-2 d}$ es el Jacobiano de la transformación. Dado que $Y$ y $x$ son independientes, resulta $f_{Y / x}(\cdot)=f_{Y}(\cdot)$. Reemplazando por las expresiones (3.15) y (3.15), resulta:

$$
\begin{aligned}
f_{Z}(Z) & =\int_{0}^{\infty} x^{-2 d} \frac{n^{n d}|Z / x|^{n-d}}{h(n, d)|\Sigma|^{n}} \exp \left(-n \operatorname{tr}\left(\Sigma^{-1} Z / x\right)\right) \frac{\gamma^{-\alpha} x^{\alpha-1}}{\Gamma(-\alpha)} \exp \left(-\frac{\gamma}{x}\right) d x \\
& =\frac{n^{n d} \gamma^{-\alpha}|Z|^{n-d}}{h(n, d)|\Sigma|^{n} \Gamma(-\alpha)} \int_{0}^{\infty} x^{\alpha-n d-1} \exp \left[-\left(n \operatorname{tr}\left(\Sigma^{-1} Z\right)+\gamma\right) x^{-1}\right] d x \\
& =\frac{n^{n d} \gamma^{-\alpha}|Z|^{n-d}}{h(n, d)|\Sigma|^{n} \Gamma(-\alpha)} \int_{0}^{\infty} x^{a} \exp \left[-b x^{-1}\right] d x
\end{aligned}
$$

En la última expresión se reemplazó $a=\alpha-n d-1$ y $b=n \operatorname{tr}\left(\Sigma^{-1} Z\right)+\gamma$.

Haciendo el cambio de variables $x=u^{-1}$ se obtiene

$$
f_{Z}(Z)=\frac{n^{n d} \gamma^{-\alpha}|Z|^{n-d} b^{a+1}}{h(n, d)|\Sigma|^{n} \Gamma(-\alpha)} \int_{0}^{\infty} u^{-a-2} \exp (-u) d u
$$

La integral en la última expresión es igual a la función Gamma $\Gamma(-a-1)$. Por último, reemplazando las expresiones de $a$ y $b$ resulta

$$
f_{Z}(Z)=\frac{n^{n d} \gamma^{-\alpha}|Z|^{n-d} \Gamma(n d-\alpha)}{h(n, d)|\Sigma|^{n} \Gamma(-\alpha)}\left(n \operatorname{tr}\left(\Sigma^{-1} Z\right)+\gamma\right)^{\alpha-n d}
$$

que es exactamente la Ec. (3.17). 


\section{Capítulo 4}

\section{Clasificación para datos homogéneos}

\subsection{Introducción}

En el contexto de procesamiento de datos SAR la clasificación de imágenes es un tópico de gran actividad e importancia (por ej., Uhlmann y Kiranyaz, 2014; Dabboor y Shokr, 2013; Sánchez-Lladó et al., 2011). Consiste básicamente en transformar la imagen de una escena a una nueva imagen, donde los píxeles están organizados en grupos. Cada grupo está definido por una característica específica que comparten los datos que pertenecen a dicho grupo. Esta característica revela información estructural de la escena subyacente que es la que desea recuperarse. En este contexto, definimos un clasificador como un algoritmo que clasifica cada píxel en una imagen, indicando a cuál de los grupos pertenece.

Como se ha visto en el Cap.3, debido a la naturaleza coherente de la señal de radar, las imágenes SAR son afectadas por speckle. Este fenómeno se produce por la superposición de las ondas reflejadas en los elementos irregulares situados a diferentes ángulos de incidencia respecto al de la onda incidente. El tamaño de estos elementos es comparable con la longitud de onda de la onda incidente (Lee y Pottier, 2009, ch.4), lo que causa superposición constructiva y destructiva que afecta a los datos enfocados. Debido a este efecto, las señales de amplitud e intensidad de la señal compleja tienden a tener muy baja relación señal a ruido (entendida como la relación entre la media y la desviación estándar) y la clasificación se vuelve una 
tarea dificultosa. Por lo tanto, la mayoría de los algoritmos de clasificación utiliza datos de intensidad o amplitud, ya que pueden ser promediados para incrementar el número equivalente de observaciones (looks) y por lo tanto mejorar la relación señal a ruido. Como contraparte, el proceso de promediado degrada la resolución y pueden perderse detalles de la imagen.

Por ejemplo, en Cloude y Pottier (1997) se propone un método de clasificación no supervisado basado en la descomposición polarimétrica del objetivo. Esta descomposición permite agrupar los píxeles de acuerdo al mecanismo de reflexión principal (superficial, dipolo o múltiples reflexiones) y al grado de aleatoriedad (entropía). En Lee et al. (1999) se desarrolla otro método no supervisado basado en la matriz de covarianza del vector de objetivo y su función de densidad de probabilidad Wishart. Este último algoritmo utiliza el esquema propuesto en Lee et al. (1999) como inicialización y luego una clasificación iterativa hasta alcanzar la convergencia. En Fjortoft et al. (1999) la segmentación se produce mediante un proceso de detección de bordes utilizando datos complejos, pero no se utiliza información de polarización. Todos estos métodos requieren promediado de datos para tener resultados confiables.

Métodos basados en el algoritmo Esperanza - Maximización (Dempster et al., 1977) también han sido propuestos para resolver el problema de clasificación. En Horta et al. (2008b) el algoritmo EM Estocástico (SEM, Stochastic EM) se utiliza en conjunto con el modelo $\mathcal{G}_{P}^{0}$ (Freitas et al., 2005) para describir terrenos homogéneos, heterogéneos y extremadamente heterogéneos. Más recientemente, en Kayabol y Zerubia (2013) se propone un método no supervisado de clasificación basado en el algoritmo Esperanza - Clasificación - Maximización (CEM, Classification EM), donde se utiliza un modelo de mezcla de distribuciones para describir datos de amplitud multilook. En Yuan et al. (2006) y Dutta y Sarma (2014) se emplea el método EM con clasificación MAP junto con un modelo de mezcla de gaussianas para datos de amplitud. Esta clasificación asigna un píxel a una determinada clase cuando la probabilidad a posteriori de que dicho píxel pertenezca a esa clase es mayor que la respectiva al resto de las clases.

En la mayoría de los trabajos que tratan con modelos de mezcla de densidades gaussianas para clasificación, los mismos son utilizados para describir variables 
reales (amplitud o intensidad), debido a que estos datos brindan la posibilidad de mejorar la relación señal a ruido por medio del promediado. Por otro lado, promediar en el dominio complejo no tiene efecto en el speckle por que no se incrementa el número equivalente de observaciones. Además, en aquellos enfoques no se optimiza la complejidad del modelo: el número de componentes de la mezcla es predefinido o estimado mediante zonas de entrenamiento en la imagen. Esto requiere información adicional del terreno o la interpretación de información que puede no estar realmente presente en los datos bajo estudio.

En este apartado se propone un algoritmo de clasificación no supervisado utilizando un modelo de mezcla de densidades gaussianas complejas para los datos SAR polarimétricos. Este algoritmo no requiere información previa o la definición de zonas de entrenamiento, y la complejidad del modelo se estima con un proceso iterativo. Esto permite representar a los datos con mezclas de densidades con el mínimo número de componentes. El algoritmo estima los parámetros de cada componente de la mezcla y luego clasifica cada píxel de acuerdo a un criterio MAP. Se divide el procedimiento en cuatro etapas: las primeras dos realizan la inicialización y la estimación de la complejidad del modelo utilizando el algoritmo EM y el Criterio de Información Bayesiano (BIC). La tercera etapa realiza la clasificación basada en el algoritmo CEM utilizando como inicialización la información provista por la etapa anterior. Finalmente, el último paso aplica un filtro no lineal para suavizar la imagen resultante.

Vale la pena mencionar que aunque se utiliza el vector de objetivo como dato complejo, no se utiliza la matriz de dispersión relacionada para realizar la clasificación ya que no es útil para describir objetivos extendidos. El método propuesto se basa en los parámetros de la mezcla de densidades gaussianas como descriptores de cada clase, incluyendo la matriz de covarianza. Esta matriz es un adecuado y extensamente utilizado descriptor de clases ya que condensa las características polarimétricas de blancos extendidos.

El algoritmo propuesto es evaluado utilizando datos simulados y reales mostrando un alto desempeño, aún cuando no se aplica ninguna técnica de reducción de speckle, con el objeto de preservar la resolución y simplicidad del modelo. Adicio- 
nalmente, la etapa de selección de modelo permite expresar el conjunto de datos con mínima complejidad preservando la estructura original, que es una característica no compartida con algoritmos convencionales.

\subsection{Modelo de Datos}

En un sistema SAR polarimétrico, cada punto en el terreno es representado por la matriz de dispersión compleja $S \in \mathbb{C}^{2 \times 2}$, cuyos elementos indican la relación entre los campos incidentes y reflejados para cada polarización (Lee y Pottier, 2009, cap.3). Los elementos en $S$ son acomodados en el llamado vector de objetivo $\boldsymbol{k}=$ $\left[\begin{array}{llll}s_{h h} & s_{h v} & s_{v h} & s_{v v}\end{array}\right]^{T}$ (el superíndice $T$ indica transposición), que es la expresión de un dato SAR SLC complejo. Suponiendo que la superficie del terreno alcanzada por la huella de la antena SAR está formada por areas homogéneas, el modelo gaussiano puede ser utilizado (Frery et al., 2012). Debido a que los datos contienen información de una porción grande de terreno, debe modelarse más de un area con diferentes características polarimétricas. Por lo tanto, se asigna un modelo de mezcla de densidades gaussianas (CGMM) a los datos, donde cada componente de la mezcla representa una característica particular de la escena subyacente.

Denotando con $\boldsymbol{k} \in \mathbb{C}^{d}$ la variable aleatoria que representa un dato SAR polaritmétrico, la forma general del modelo de mezcla con $K$ clases es la siguiente:

$$
f(\boldsymbol{k} ; \boldsymbol{\theta})=\sum_{j=1}^{K} \omega_{j} f_{j}\left(\boldsymbol{k} ; \boldsymbol{\theta}_{j}\right),
$$

donde $f_{1}, \ldots, f_{K}$ son las $K$ densidades de probabilidad del modelo representando cada una a una clase. Cada $f_{j}$ tiene un conjunto de parámetros descripto por el vector $\boldsymbol{\theta}_{j}$. Los coeficientes $w_{j}$ indican la proporción de la $j$-ésima componente en la mezcla,

sujetas a la restricciones $\sum_{j=1}^{K} \omega_{j}=1 \mathrm{y} \omega_{j} \geq 0, j=1 \ldots K$. El vector de parámetros de la mezcla es $\boldsymbol{\theta}=\left(\omega_{1}, \ldots, \omega_{K}, \boldsymbol{\theta}_{1}, \ldots, \boldsymbol{\theta}_{K}\right)$. Bajo las condiciones gaussianas cada término de (4.1) tiene la forma $f_{j}\left(\boldsymbol{k} ; \boldsymbol{\theta}_{j}\right)=\pi^{-d}\left|\Sigma_{j}\right|^{-1} \exp \left(-\left(\boldsymbol{k}-\boldsymbol{\mu}_{j}\right)^{H} \Sigma_{j}^{-1}\left(\boldsymbol{k}-\boldsymbol{\mu}_{j}\right)\right)$, donde la media $\boldsymbol{\mu}_{j} \in \mathbb{C}^{d}$ y covarianza $\Sigma_{j} \in \mathbb{C}^{d \times d}$ son los parámetros $\boldsymbol{\theta}_{j}$ de la $j$-ésima clase. 
El objetivo del algoritmo de clasificación propuesto es identificar todas las clases presentes en el conjunto de datos, por medio de la estimación del orden de modelo $K$ y los parámetros de la mezcla $\boldsymbol{\theta}$ que mejor se ajustan a los datos en el sentido de máxima verosimilitud. Cada par $\left(\omega_{j}, \boldsymbol{\theta}_{j}\right)$ describe una clase y a su vez identifica una característica polarimétrica de la superficie. Debido a la complejidad de modelo, no existe solución analítica para este problema de optimización. Por lo tanto, se utiliza un enfoque iterativo basado en el algoritmo EM para determinar el orden del modelo y estimar los parámetros.

\subsection{Estructura del algoritmo}

En esta sección se describe en detalle la estructura y funcionamiento del algoritmo, el cual está organizado en las siguientes cuatro etapas:

\section{Inicialización}

2. Selección del modelo

\section{Refinamiento}

\section{Suavizado}

Como se señaló previamente, el algoritmo procede iterativamente. Luego de una etapa de inicialización, la selección de orden de modelo se realiza en base al Criterio de Información Bayesiano -BIC- (Schwarz, 1978) y el algoritmo EM. Esta etapa provee no sólo el orden del modelo, sino también una inicialización apropiada para la etapa de refinamiento. Con la clasificación inicial como punto de partida y el algoritmo CEM (Celeux y Govaert, 1992), se obtiene la clasificación final y la estimación de los parámetros. Finalmente, un filtro de moda se aplica en la imagen clasificada para suavizar la imagen final. En las siguientes secciones se detallará el funcionamiento de cada etapa. En Algoritmo 1 se presenta el pseudocódigo del algoritmo. 


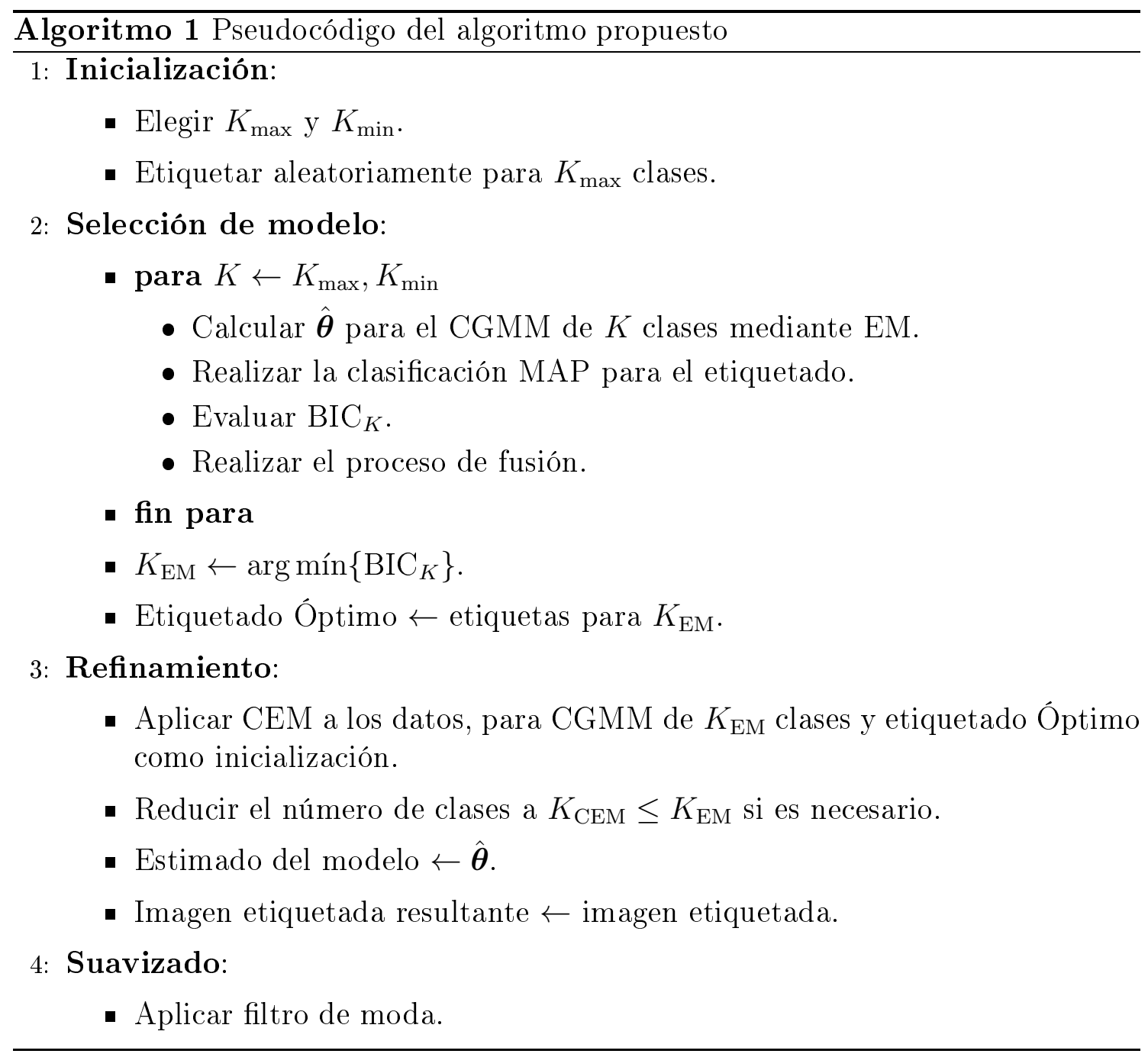




\subsubsection{Inicialización}

Inicialmente, se eligen un máximo y un mínimo número de clases, $K_{\max }$ y $K_{\min }$. A pesar de que es recomendable que esta elección se haga en base a información previa del terreno, esto no es un requerimiento ya que puede elegirse $K_{\min }=1 \mathrm{y}$ $K_{\max }$ igual a la cantidad de datos. Sin embargo, esto resulta muy costoso en términos de memoria y tiempo de cómputo.

Siendo un algoritmo no supervisado, no se requiere etapas de entrenamiento. Inicialmente hay $K_{\max }$ clases diferentes. Cada dato es aleatoriamente asignado a una clase estableciendo su etiqueta a cualquiera de las $K_{\max }$ clases.

Aunque arbitrario, este simple esquema de inicialización es suficiente para que el algoritmo alcance la convergencia, haciendo innecesario un procedimiento de inicialización más complicado.

\subsubsection{Selección del modelo}

Dado un conjunto de datos SAR, interesa describirlo con un CGMM de mínima complejidad que mantenga toda la información relevante. Es decir, se debe determinar el mínimo número de clases (lo que supone el mínimo número de parámetros) que se ajusta al conjunto de datos. Este proceso, llamado selección de modelo, se realiza iterativamente: se estiman los parámetros para las $K$ clases y se evalúa el ajuste del modelo a los datos. Luego, se reduce el número de clases a $K-1$ y se re-

calcula el ajuste. Esto se repite desde $K_{\max }$ a $K_{\min }$, utilizando el algoritmo EM para la estimación de parámetros y BIC para evaluar el ajuste. El modelo que minimiza el BIC es seleccionado. La reducción del número de clases se realiza fusionando dos clases clandidatas en una sola, en cada paso. En esta sección se describe los fundamentos del algoritmo EM, el cálculo de BIC, la selección de las clases candidatas y el proceso de fusión.

\section{El algoritmo EM}

El algoritmo EM soluciona el problema de estimación de máxima verosimilitud utilizando un enfoque iterativo de dos etapas: la primera donde el número de va- 
riables se reduce mediante promediado estadístico, de modo que en la segunda el problema de maximización original se convierte en uno más sencillo de resolver.

Debido a la complejidad del modelo de mezclas gaussiano, no existe solución con expresión cerrada para la estimación de máxima verosmilitud de los parámetros $\boldsymbol{\theta}$. En lugar de ello, EM maximiza la función $Q\left(\boldsymbol{\theta} / \boldsymbol{\theta}^{\prime}\right)=$ $\sum_{i=1}^{N} E_{\mathbf{y}_{i} \mid \boldsymbol{k}_{i} ; \boldsymbol{\theta}^{\prime}}\left[\log \left(f_{\boldsymbol{k}_{i}, \mathbf{y}_{i} ; \boldsymbol{\theta}^{\prime}}\left(\boldsymbol{k}_{i}, \mathbf{y}_{i} ; \boldsymbol{\theta}\right)\right)\right]$ en cada iteración, con el mínimo error cuadrático medio. En la última expresión $\boldsymbol{\theta}^{\prime}$ es el estimado del vector de parámetros calculado en la iteración previa, $f_{\boldsymbol{k}_{i}, \mathbf{y}_{i} ; \boldsymbol{\theta}}(\cdot)$ es la función de densidad conjunta de los datos completos (Dempster et al., 1977) y $E_{\mathbf{y}_{i} \mid \boldsymbol{k}_{i} ; \boldsymbol{\theta}^{\prime}}[\cdot]$ es el operador esperanza condicional. Puede demostrarse que la secuencia $\boldsymbol{\theta}^{(l)}$ hace crecer la función de verosimilitud tal que cuando se alcanza la convergencia (entendida como cambios suficientemente pequeños en la función de verosimilitud) en la iteración $L$-ésima, resulta $\boldsymbol{\theta}^{(L)}=\hat{\boldsymbol{\theta}}$ (McLachlan y Krishnan, 2008, p. 81).

El algoritmo EM consiste en dos pasos principales:

1) Paso E: Calcular $Q\left(\boldsymbol{\theta} / \boldsymbol{\theta}^{(l-1)}\right)$ usando el estimado previo $\boldsymbol{\theta}^{(l-1)}$.

2) Paso M: Calcular

$$
\boldsymbol{\theta}^{(l)}=\arg \operatorname{máx}_{\boldsymbol{\theta}} Q\left(\boldsymbol{\theta} / \boldsymbol{\theta}^{(l-1)}\right)
$$

La estructura del proceso iterativo es el siguiente:

a) Inicializar el algoritmo apropiadamente, de manera que el primer estimado $\boldsymbol{\theta}^{(0)}$ pueda ser calculado. En el algoritmo presentado, $\boldsymbol{\theta}^{(0)}$ se calcula en base a la asignación de píxeles en la etapa de inicialización.

b) Repetir los pasos M y E hasta que

$$
\ln \left(L\left(\boldsymbol{\theta}^{(l)} ; \boldsymbol{k}_{1}, \ldots, \boldsymbol{k}_{N}\right)\right)-\ln \left(L\left(\boldsymbol{\theta}^{(l-1)} ; \boldsymbol{k}_{1}, \ldots, \boldsymbol{k}_{N}\right)\right) \leq \epsilon
$$

Suponiendo el modelo descripto en (4.1), la iteración l-ésima resulta: 
- Paso E:

$$
\begin{aligned}
Q\left(\boldsymbol{\theta} / \boldsymbol{\theta}^{(l-1)}\right) & =\sum_{i=1}^{N} \sum_{j=1}^{K} \gamma_{i j}^{(l)} \log \left(\omega_{j} f_{j}\left(\boldsymbol{k}_{i}, \boldsymbol{\theta}_{j}\right)\right), \\
\gamma_{i j}^{(l)} & =\frac{\omega_{j}^{(l-1)} f_{j}\left(\boldsymbol{k}_{i}, \boldsymbol{\theta}_{j}^{(l-1)}\right)}{\sum_{r=1}^{K} \omega_{r}^{(l-1)} f_{r}\left(\boldsymbol{k}_{i}, \boldsymbol{\theta}_{r}^{(l-1)}\right)} .
\end{aligned}
$$

- Paso M:

$$
\begin{aligned}
\omega_{j}^{(l)} & =\frac{\sum_{i=1}^{N} \gamma_{i j}^{(l)}}{N}, \\
\boldsymbol{\mu}_{j}^{(l)} & =\frac{\sum_{i=1}^{N} \gamma_{i j}^{(l)} \boldsymbol{k}_{i}}{\sum_{i=1}^{N} \gamma_{i j}^{(l)}}, \\
\Sigma_{j}^{(l)} & =\frac{\sum_{i=1}^{N} \gamma_{i j}^{(l)}\left(\boldsymbol{k}_{i}-\boldsymbol{\mu}_{j}^{(l)}\right)\left(\boldsymbol{k}_{i}-\boldsymbol{\mu}_{j}^{(l)}\right)^{H}}{\sum_{i=1}^{n} \gamma_{i j}^{(l)}} .
\end{aligned}
$$

La Ec. (4.3) calcula la probabilidad a posteriori de que el dato $i$ pertenezca a $j$ ésima clase, en la iteración $l$. Las Ecs. (4.4) a (4.6) son el resultado de la maximización para cada parámetro en la iteración l-ésima.

\section{Clasificación MAP}

Después de que EM alcanza la convergencia se realiza la clasificación MAP. Siendo $L$ la iteración en que converge EM, entonces para cada dato $i$, se analiza $\gamma_{i j}^{(L)}$ para todas las $K$ clases. La clasificación se realiza asignando (etiquetando) el $i$-ésimo dato a la $k$-ésima clase si $\gamma_{i k}^{(L)}>\gamma_{i j}^{(L)}$, para $k \neq j, j=1, \ldots, K$.

Luego del paso de inicialización donde cada píxel fue asignado a una de las $K_{\max }$ clases, el primer estimado $\boldsymbol{\theta}^{(0)}$ para $K_{\max }$ clases puede calcularse utilizando (4.4) a (4.6) (en este punto, $\gamma_{i j}$ se comporta como una función indicadora: $\gamma_{i j}=1$ si el píxel $i$ pertenece a la clase $j, \mathrm{y} \gamma_{i j}=0$ en caso contrario), permitiendo a EM comenzar su proceso iterativo. Una vez alcanzada la convergencia luego de la iteración $L_{K_{\max }}$, se obtiene el estimado $\boldsymbol{\theta}^{\left(L_{K_{\max }}\right)}$, es decir, el CGMM está caracterizado completamente 
para $K_{\max }$ clases. Además, cada dato queda etiquetado indicando a qué clase fue asignado.

Después de la evaluación del modelo mediante BIC, se aplica un proceso de fusión (explicado luego) para reducir el número de clases en uno. Se calcula un nuevo vector $\boldsymbol{\theta}$ para $K_{\max }-1$ clases, basado en $\boldsymbol{\theta}^{\left(L_{K_{\max }}\right)}$. Luego se aplica EM nuevamente con este nuevo vector $\boldsymbol{\theta}$ como inicialización para calcular el CGMM para las $K_{\max }-1$ clases. Este proceso se repite hasta que se alcanza $K_{\min }$ clases. De esta manera resulta una secuencia $\left\{\mathrm{BIC}_{K}\right\}, K=K_{\max }, \ldots, K_{\min }$ y una clasificación (datos etiquetados) para cada valor de $K$. El orden del modelo es entonces seleccionado como el $K$ para el cual $\left\{\mathrm{BIC}_{K}\right\}$ es mínimo, y se lo denota $K_{\mathrm{EM}}$. Los datos etiquetados y $K_{\mathrm{EM}}$ son utilizados como inicialización en el paso de refinamiento.

\section{Criterio de Información Bayesiano}

Dado el conjunto de datos SAR y el CGMM propuesto, resulta de interés cuántas componentes gaussianas son necesarias para describirlo. Desde el punto de vista de la función de verosimilitud, cuantas más componentes contenga el modelo, mejor se ajustará éste a los datos, ya que su valor máximo aumenta. Sin embargo, demasiadas componentes pueden sobreestimar el número de características diferentes del terreno. BIC evalúa la probabilidad a posteriori de que los datos hayan sido generados por un modelo específico, dados los datos observados. Esto resulta en una expresión donde la función de verosimilitud logarítmica se penaliza por un término que tiene en cuenta el número de parámetros a estimar (Konishi y Kitawa, 2008). En consecuencia, el modelo óptimo seleccionado para los datos observados es el que maximiza dicha probabilidad a posteriori. BIC se define como el logaritmo natural de dicha probabilidad multiplicado por -2 , de manera que se transforma en un problema de minimización.

La etapa de selección de modelo proporciona $K_{\max }-K_{\min }+1$ diferentes modelos, cada uno descripto por su propio vector de parámetros. Para evaluar el ajuste del modelo a los datos, BIC se calcula como

$$
\mathrm{BIC}=-2 \mathcal{L}\left(\boldsymbol{\theta} ; \boldsymbol{k}_{1}, \ldots, \boldsymbol{k}_{N}\right)+p \ln (N)
$$


donde $\mathcal{L}\left(\boldsymbol{\theta} ; \boldsymbol{k}_{1}, \ldots, \boldsymbol{k}_{N}\right)$ es la función de verosimilitud logarítmica, $\boldsymbol{\theta}$ es el vector de parámetros y $p$ es la dimensión. En este caso, $\mathcal{L}\left(\boldsymbol{\theta} ; \boldsymbol{k}_{1}, \ldots, \boldsymbol{k}_{N}\right)$ es el logaritmo natural del producto $\prod_{i=1}^{N} f\left(\boldsymbol{k}_{i} ; \boldsymbol{\theta}\right)$. En un CGMM con $K$ clases y datos $\boldsymbol{k} \in \mathbb{C}^{d}$, hay $K$ matrices de covarianza Hermíticas de dimensión $d \times d, K$ vectores de media de $d$ elementos y $K$ factores de peso $\omega_{j}$. Esto resulta en $p=\left(2 d^{2}-d+1\right) K-1$ parámetros reales. En este caso, $d=3$, entonces $p=16 K-1$.

\section{Fusión de clases}

Durante la etapa de selección de modelo, el número de clases debe ser reducido progresivamente. Después de completar cada ciclo de estimación EM, se seleccionan y fusionan dos clases combinando sus parámetros (factores de peso, medias y matrices de covarianza) para formar una nueva clase. Esta nueva clase y las restantes que no participaron del proceso de fusión, conforman el modelo reducido utilizado como inicialización por el siguiente ciclo de estimación EM. Con el objeto de no afectar drásticamente el ajuste logrado en la etapa previa, las clases a fusionar deben ser elegidas de manera que el nuevo CGMM mantenga la mayor la similitud estadística posible con el previo CGMM. Para esta tarea se utiliza el método propuesto en Runnalls (2007), donde el autor trata el problema de fusionar dos componentes gaussianas de una mezcla utilizando la divergencia de Kullback-Leibler (KL). Por completitud, seguidamente se explica los fundamentos del método.

Para enfatizar su dependencia con cada parámetro, se denotará con $\left(\omega_{i}, \boldsymbol{\mu}_{i}, \Sigma_{i}\right)$ y $\left(\omega_{j}, \boldsymbol{\mu}_{j}, \Sigma_{j}\right)$ a las componentes $f_{i}\left(\boldsymbol{k} ; \boldsymbol{\theta}_{i}\right)$ y $f_{j}\left(\boldsymbol{k} ; \boldsymbol{\theta}_{j}\right)$ de $(4.1)$, respectivamente. La fusión de las componentes $i$ y $j$ resultan en una nueva componente gaussiana $f_{i j}\left(\boldsymbol{k} ; \boldsymbol{\theta}_{i j}\right)$, cuyos parámetros $\left(\omega_{i j}, \boldsymbol{\mu}_{i j}, \Sigma_{i j}\right)$ deben determinarse. Una manera adecuada de conseguirlo es manteniendo los momentos de orden cero, uno y dos con los que ambas componentes contribuyen a la mezcla, de la siguiente manera: 


$$
\begin{aligned}
& \omega_{i j}=\omega_{i}+\omega_{j}, \\
& \boldsymbol{\mu}_{i j}=\omega_{i} \boldsymbol{\mu}_{i}+\omega_{j} \boldsymbol{\mu}_{j}, \\
& \Sigma_{i j}=\frac{1}{\omega_{i j}}\left[\omega_{i} \Sigma_{i}+\omega_{j} \Sigma_{j}+\frac{\omega_{i} \omega_{j}}{\omega_{i j}}\left(\boldsymbol{\mu}_{i}-\boldsymbol{\mu}_{j}\right)\left(\boldsymbol{\mu}_{i}-\boldsymbol{\mu}_{j}\right)^{H}\right] .
\end{aligned}
$$

Por otro lado, una forma natural de calcular la similitud estadística (o la falta de ella) entre dos distribuciones, es la divergencia KL. Si $f_{1}(\boldsymbol{k})$ y $f_{2}(\boldsymbol{k})$ son dos funciones de densidad de probabilidad en $R^{d}$, la divergencia KL de $f_{2}(\boldsymbol{k})$ desde $f_{1}(\boldsymbol{k})$ se define como:

$$
d_{K L}\left(f_{1}, f_{2}\right)=\int_{R^{d}} f_{1}(\boldsymbol{k}) \log \frac{f_{1}(\boldsymbol{k})}{f_{2}(\boldsymbol{k})} d \boldsymbol{k} .
$$

Claramente $d_{K L}\left(f_{1}, f_{2}\right) \geq 0$, y $d_{K L}\left(f_{1}, f_{1}\right)=0$. Cuanto mayor es $d_{K L}\left(f_{1}, f_{2}\right)$, menos similares son $f_{1}(\boldsymbol{k})$ y $f_{2}(\boldsymbol{k})$. Cuando $f_{1}(\boldsymbol{k}) \sim C N\left(\mu_{1}, \Sigma_{1}\right)$ and $f_{2}(\boldsymbol{k}) \sim$ $C N\left(\mu_{2}, \Sigma_{2}\right)$ son densidades gaussianas complejas, la divergencia KL tiene la expresión cerrada:

$$
\begin{aligned}
d_{K L}\left(f_{1}, f_{2}\right)= & \frac{1}{2} \operatorname{tr}\left[\Sigma_{2}^{-1}\left(\Sigma_{1}+\Sigma_{2}+\left(\mu_{1}-\mu_{\mathbf{2}}\right)\left(\mu_{1}-\mu_{2}\right)^{H}\right)\right] \\
& +\frac{1}{2} \log \frac{\left|\Sigma_{2}\right|}{\left|\Sigma_{1}\right|} .
\end{aligned}
$$

En el caso de CGMM, no existe forma cerrada para la expresión de la divergencia KL. Sin embargo, en Runnalls (2007) se calcula una cota superior para la divergencia del modelo CGMM. Esta cota provee una medida de similitud entre modelos CGMM antes y después de la fusión. En el citado trabajo, el autor muestra que al fusionar las componentes $f_{i}\left(\boldsymbol{k} ; \boldsymbol{\theta}_{i}\right)$ y $f_{j}\left(\boldsymbol{k} ; \boldsymbol{\theta}_{j}\right)$ de la muestra para formar un CGMM reducido, la divergencia KL del nueva CGMM con respecto al original está acotada, y la cota puede calcularse. En otras palabras, la cota superior de la divergencia KL de la mezcla luego de la fusión respecto de la mezcla antes de la fusión de $f_{i}\left(\boldsymbol{k} ; \boldsymbol{\theta}_{i}\right)$ y $f_{j}\left(\boldsymbol{k} ; \boldsymbol{\theta}_{j}\right)$, denotada $B_{\text {div }}\left(\left(\omega_{i}, \boldsymbol{\mu}_{i}, \Sigma_{i}\right),\left(\omega_{j}, \boldsymbol{\mu}_{j}, \Sigma_{j}\right)\right)$, resulta: 


$$
\begin{aligned}
& B_{d i v}\left(\left(\omega_{i}, \boldsymbol{\mu}_{i}, \Sigma_{i}\right),\left(\omega_{j}, \boldsymbol{\mu}_{j}, \Sigma_{j}\right)\right) \\
& \quad=\frac{1}{2}\left[\left(\omega_{i}+\omega_{j}\right) \log \left|\Sigma_{i j}\right|-\omega_{i} \log \left|\Sigma_{i}\right|-\omega_{j} \log \left|\Sigma_{j}\right|\right] .
\end{aligned}
$$

Esta expresión, que resulta independiente de las medias, provee un método para elegir las componentes a ser fusionadas. Evaluando (4.13) para cada par de componentes en la mezcla, se eligen los componentes $i$ y $j$ tales que $B_{d i v}\left(\left(\omega_{i}, \boldsymbol{\mu}_{i}, \Sigma_{i}\right),\left(\omega_{j}, \boldsymbol{\mu}_{j}, \Sigma_{j}\right)\right)$ es mínimo. Los parámetros del nuevo componente estarán dados por las Ecs. (4.8) a $(4.10)$.

\subsubsection{Refinamiento}

Luego de la etapa de selección de modelo, se aplica un proceso de refinamiento para mejorar la clasificación. Un método basado en el algoritmo EM, denominado CEM, se aplica a los datos tomando como inicialización los datos etiquetados para las $K_{\text {EM }}$ clases calculadas en la etapa anterior.

El algoritmo CEM se basa en los mismos principios que EM, pero incorpora un paso de clasificación (paso C) entre los pasos E y M. Después de calcular (4.3) en el paso $\mathrm{E}$, el paso $\mathrm{C}$ consiste en asignar el $i$-ésimo dato a la $k$-ésima clase si $\gamma_{i k}>\gamma_{i j}$, para $k \neq j, j=1, \ldots, K$. Es decir, consiste en realizar la clasificación MAP inmediatamente después del paso E en cada iteración. La diferencia respecto de EM convencional consiste en que los parámetros de cada clase calculados en el paso M son estimados utilizando los datos pertenecientes a esa clase en particular, como sigue: 


$$
\begin{aligned}
\omega_{j}^{(l)} & =\frac{N_{j}^{(l)}}{N}, \\
\boldsymbol{\mu}_{j}^{(l)} & =\frac{\sum_{\boldsymbol{k} \in \text { clase } j} \boldsymbol{k}}{N_{j}^{(l)}}, \\
\Sigma_{j}^{(l)} & =\frac{\sum_{\boldsymbol{k} \in \text { clase } j}\left(\boldsymbol{k}-\boldsymbol{\mu}_{j}^{(l)}\right)\left(\boldsymbol{k}-\boldsymbol{\mu}_{j}^{(l)}\right)^{H}}{N_{j}^{(l)}},
\end{aligned}
$$

donde $N_{j}^{(l)}=\#\{\boldsymbol{k} \in$ clase $j\}$ es el número de datos pertenecientes a la clase $j$ en la iteración $l$. Puede demostrarse (Celeux y Govaert (1992), Prop. 2) que CEM converge a un punto crítico de la función de verosimilitud si los parámetros están bien definidos. Y más aún, puede alcanzar el máximo siendo inicializado con los parámetros estimados por EM de la etapa previa.

En la mayoría de los casos luego de aplicar CEM, se observó que algunas de las $K_{\mathrm{EM}}$ clases contenían un número bajo de píxeles, indicando la necesidad de una reducción adicional en el número de clases. La razón es que, al contrario que en el proceso de fusión, la clasificación CEM no contempla la estructura global de los datos. Para evitar las clases no relevantes, se permite que CEM reduzca el número de clases como sigue. Si el número de miembros de una clase se vuelve no significante en cualquier paso de la clasificación, sus píxeles son reasignados aleatoriamente al resto de las clases, se reduce acordemente el número de clases y la estimación continúa con el nuevo número de clases. Se declara que el número de miembros de una clase es no significante cuando es menor a veinte veces el número de parámetros a estimar para esa clase. Esto evita tener que estimar los parámetros de una clase sobre la que no se tiene suficiente información.

Luego de alcanzar la convergencia, CEM proporciona los factores de peso, medias y matrices de covarianza estimados de las $K_{\mathrm{CEM}} \leq K_{\mathrm{EM}}$ clases del CGMM, junto con la clasificación (datos etiquetados) utilizada para mostrar gráficamente el resultado en una imagen.

Dado que CEM es un algoritmo basado en EM, su uso inmediatamente después del paso de selección de modelo donde ya se había aplicado EM puede parecer 
de utilidad cuestionable. Sin embargo, los resultados obtenidos muestran que una vez establecido el orden del modelo, CEM logra mejores clasificaciones que EM convencional. Esto se debe a que CEM realiza una clasificación de los píxeles dura, mientras que EM realiza una clasificación blanda por medio del parámetro $\gamma_{i j}$. Por otro lado, CEM no podría utilizarse directamente en la etapa de selección de modelo debido a dos razones principales: es muy sensible al punto de inicialización y los estimados que provee no son los de máxima verosimilitud requeridos para el cálculo de BIC.

\subsubsection{Suavizado}

Luego de la clasificación CEM, la imagen clasificada puede aparecer ruidosa, con grupos de píxeles con etiquetas diferentes a las de los píxeles vecinos. Este efecto es una característica conocida de los algoritmos basados en MLE cuando no se utiliza la información de píxeles vecinos en el proceso de estimación. Se propone entonces aplicar un filtro no lineal de moda a los datos etiquetados, para mejorar la visualización de los resultados.

Teniendo la imagen clasificada, se define una ventana de filtrado de $h \times w$ píxeles, con $h$ y $w$ impares por conveniencia. El suavizado se realiza asignando a cada píxel en la posición $[i, j]$ el valor de la moda de las etiquetas dentro de la ventana del filtro, cuando ésta está centrada en $[i, j]$. El nuevo valor del píxel aún pertenece a $\left\{1, \ldots, K_{\mathrm{CEM}}\right\}$ debido a la operación moda, y un pequeño grupo de píxeles son re-etiquetados, creando una imagen más suave. Como contraparte, la resolución de la clasificación puede verse reducida en la frontera entre clases. El compromiso entre resolución y nivel de suavizado se resuelve heurísticamente estableciendo el tamaño de ventana, ya que ambos requerimientos son dependientes de la aplicación. En caso que dentro de la ventana existan diferentes clases con la misma cantidad de píxeles, el píxel analizado se asigna aleatoriamente a una de las clases involucradas. En este trabajo se ha utilizado $h=w=5$. 


\subsection{Desempeño con datos simulados}

En esta sección se presenta el desempeño del algoritmo propuesto cuando se lo aplica a datos simulados, y la comparación de los resultados con los obtenidos por el conocido clasificador Wishart (Lee et al., 1999). Vale la pena notar que la elección de un algoritmo para comparar resultados no es una tarea sencilla, a pesar de la gran variedad de algoritmos disponibles. Se ha elegido el algoritmo de Wishart en base a los siguientes argumentos: es ampliamente utilizado en la bibliografía, el modelo gaussiano complejo está ligado a la densidad Wishart para matrices de covarianza muestrales como las que se usan en el algoritmo propuesto y, finalmente, se trata de un algoritmo no supervisado e iterativo como el que se propone.

Debido a que los datos utilizados en esta sección son simulados, conocemos el terreno verdadero (ground truth), por lo que podemos utilizar la matriz de confusión y el estadístico kappa para comparar resultados.

\subsubsection{Datos Simulados}

Para evaluar el algoritmo, se simuló un terreno (terreno verdadero simulado) cuyos datos SLC recibidos se describen mediante la Ec. (4.1). La escena es una imagen de $200 \times 200$ píxeles formada por seis zonas con diferente características de dispersión, cada una respresentada por una componente gaussiana compleja $f_{j}\left(\boldsymbol{k} ; \boldsymbol{\theta}_{j}\right)$, $j=1, \ldots, 6$. La Fig. 4.1(a) muestra la escena simulada y la Tabla 4.1 muestra los parámetros que describen cada zona. Cada matriz de covarianza pertenece a una región diferente del plano $H / \alpha$ (Cloude y Pottier, 1997) para simular realizaciones provenientes de mecanismos de dispersion diferentes. Los factores de peso $\omega$ resultan de la proporción de píxeles pertenecientes a cada zona sobre la cantidad de píxeles total. Todas las zonas se simularon con media nula.

Debe notarse que las zonas superior e inferior de la Fig. 4.1(a) tienen el mismo color, representando el mismo tipo de terreno. Lo mismo aplica para las zonas del extremo derecho e izquierdo. La 4.1(b) es una imagen color RGB de los datos simulados SLC de la escena, donde cada color representa la contribución de cada componente polarimétrica. 


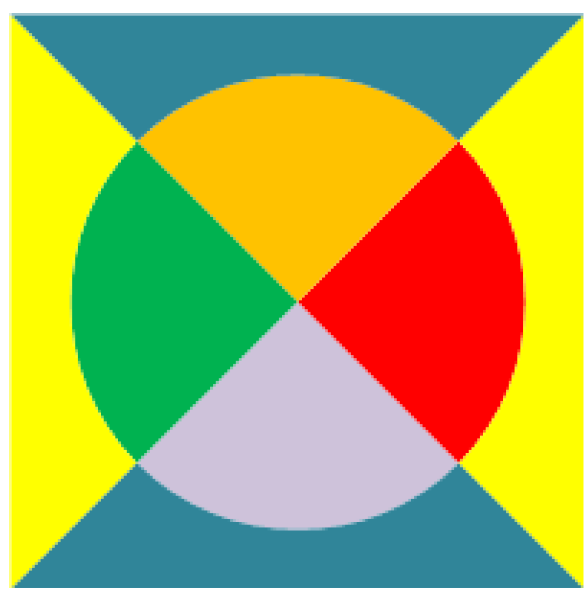

(a)

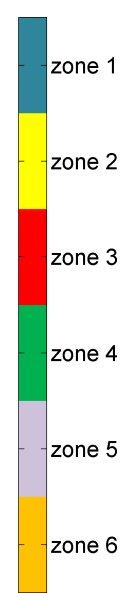

ne 6

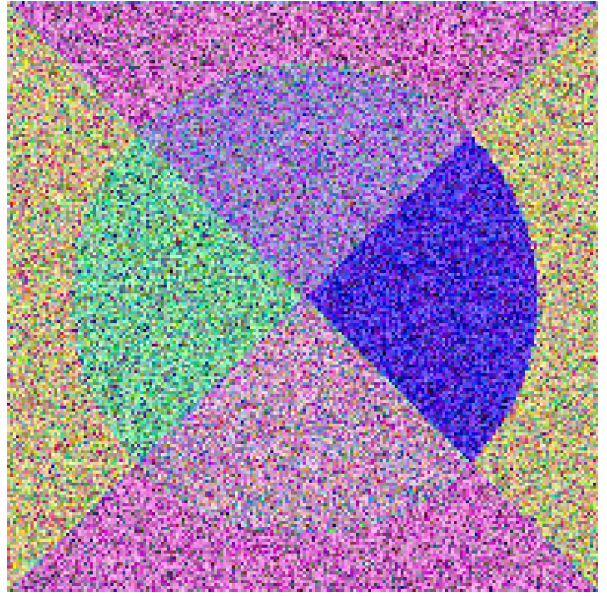

(b)

Figura 4.1: Datos Simulados. (a) Terreno verdadero. (b) Datos SLC. Rojo:|HH-VV|. Verde:|HV+VH|. Azul:|HH+VV|.

\subsubsection{Resultados}

Se aplicó el algoritmo propuesto a los datos SLC simulados, con $K_{\max }=10 \mathrm{y}$ $K_{\text {min }}=2$. La Fig. 4.2 muestra los resultados de la etapa de selección de modelo y la Fig. 4.2(a) muestra el valor BIC de acuerdo a la reducción del número de clases. Puede observarse que BIC toma el mínimo valor para $K=6$, que es el número de clases verdadero. El algoritmo establece $K_{\mathrm{EM}}=6$ como óptimo y almacena los correspondientes datos etiquetados. La Fig. 4.2(b) muestra la imagen etiquetada.

La etapa de refinamiento aplica CEM con $K_{\mathrm{EM}}=6$ a los datos etiquetados obtenidos en la etapa previa. La 4.3(a) muestra la imagen etiquetada resultante, muy similar a $4.2(\mathrm{~b})$. El número de clases no fue reducido (es decir, $K_{\mathrm{CEM}}=K_{\mathrm{EM}}=6$ ) porque la etapa anterior proveyó una inicialización muy cercana a la óptima para el algoritmo CEM, lo que produjo una poca cantidad de píxeles re-etiquetados. Finalmente, la Fig. 4.3(b) muestra el resultado final luego de la aplicación del suavizado. Como se mencionó anteriormente, se aplicó el algoritmo Wishart a los datos SLC simulados para $K=6$ e inicialización aleatoria ${ }^{1}$. En la simulaciones se utilizó una

\footnotetext{
${ }^{1}$ Se observó que el resultado final es independiente de la inicialización, debido al procesamiento de múltiples observaciones.
} 
Tabla 4.1: Parámetros del terreno simulado

\begin{tabular}{|c|c|c|c|c|c|}
\hline Zona & \multicolumn{3}{|c|}{$\Sigma$} & $\omega$ & Plano $H / \alpha$ \\
\hline \multirow{4}{*}{ Zona 1} & 0,907 & $-0,040+j 0,027$ & $0,001+j 0,169$ & \multirow{3}{*}{0,2442} & \multirow{3}{*}{ región 8} \\
\hline & $-0,040-j 0,027$ & 0,043 & $0,006-j 0,010$ & & \\
\hline & $0,001-j 0,169$ & $0,006+j 0,010$ & 0,050 & & \\
\hline & 0,339 & $0,005+j 0,253$ & $-0,141+j 0,037$ & \multirow{4}{*}{0,2512} & \multirow{4}{*}{ región 4} \\
\hline \multirow[t]{3}{*}{ Zona 2} & $0,005-j 0,253$ & 0,408 & $-0,031+j 0,119$ & & \\
\hline & {$[-0,141-j 0,037$} & $-0,031-j 0,119$ & 0,253 & & \\
\hline & 0,374 & $-0,048-j 0,044$ & $0,461-j 0,060^{=}$ & & \\
\hline \multirow[t]{3}{*}{ Zona 3} & $-0,048+j 0,044$ & 0,018 & $-0,050+j 0,062$ & \multirow[t]{2}{*}{0,1203} & \multirow[t]{2}{*}{ región 9} \\
\hline & $0,461+j 0,060$ & $-0,050-j 0,044$ & 0,609 & & \\
\hline & 0,105 & $-0,045+j 0,208$ & $0,053+j 0,029]$ & \multirow{3}{*}{0,1257} & \multirow{3}{*}{ región 7} \\
\hline \multirow[t]{3}{*}{ Zona 4} & $-0,045-j 0,208$ & 0,775 & $0,113-j 0,156$ & & \\
\hline & $0,053-j 0,029$ & $0,113+j 0,156$ & 0,120 & & \\
\hline & 0,434 & $0,218-j 0,012$ & $0,071-j 0,225]$ & & \multirow{3}{*}{ región 5} \\
\hline \multirow[t]{3}{*}{ Zona 5} & $0,218+j 0,012$ & 0,322 & $0,030-j 0,112$ & \multirow[t]{2}{*}{0,1221} & \\
\hline & $0,071+j 0,225$ & $0,030+j 0,112$ & 0,244 & & \\
\hline & 0,396 & $0,146-j 0,046$ & $0,311+j 0,002]$ & \multirow{3}{*}{0,1365} & \multirow{3}{*}{ región 6} \\
\hline \multirow[t]{2}{*}{ Zona 6} & $0,146+j 0,046$ & 0,187 & $0,126+j 0,105$ & & \\
\hline & $0,311-j 0,002$ & $0,126-j 0,105$ & 0,417 & & \\
\hline
\end{tabular}

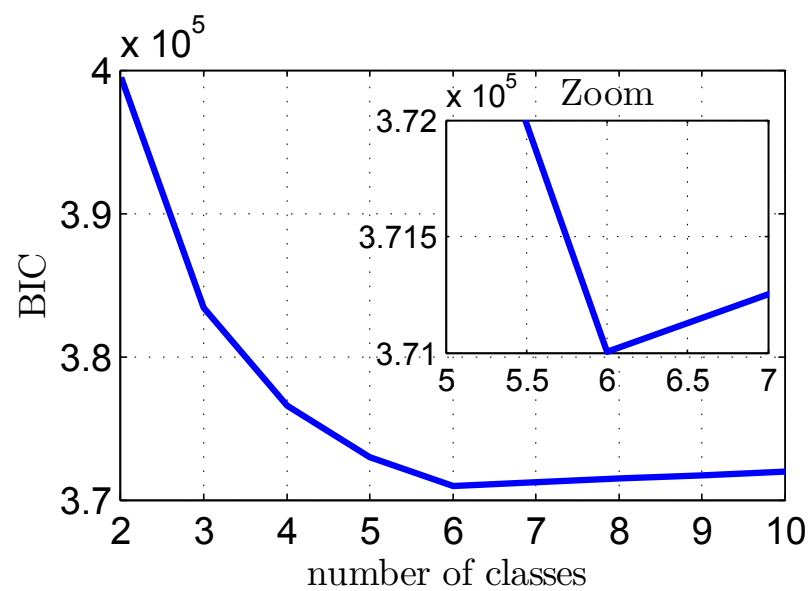

(a)

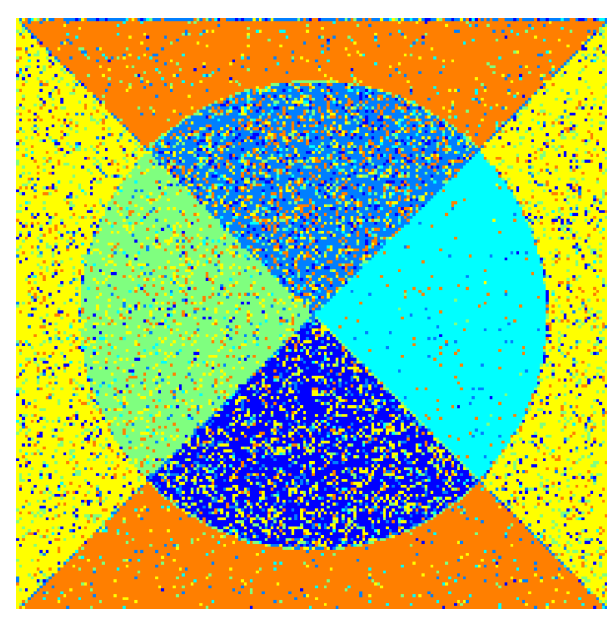

(b)

Figura 4.2: Resultado de la selección de modelo. (a) Valor BIC, (b)Imagen etiquetada para $K=6$ clases.

matriz de covarianza de 25 observaciones, obtenidas mediante el promediado de $\boldsymbol{k}^{H}$ 


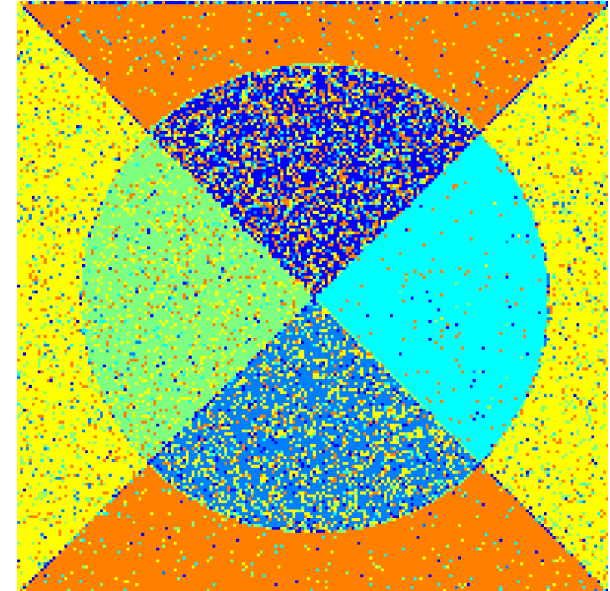

(a)

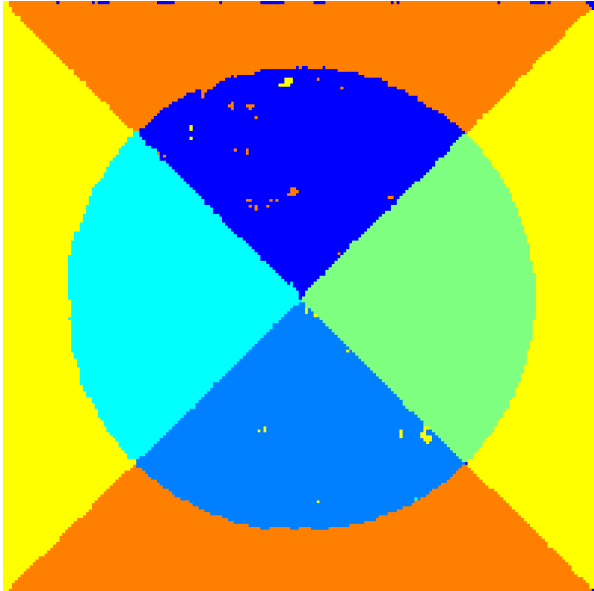

(b)

Figura 4.3: Resultados para datos simulados. (a) Refinamiento, (b) Suavizado.

con una ventana de $5 \times 5$. La Fig. 4.4 muestra la imagen etiquetada con las seis clases identificadas.

Los colores en las clasificaciones resultantes de las Figs. 4.3(b) y 4.4 no indican características particulares, sólo indican la estructura de la clasificación y el número de clases.

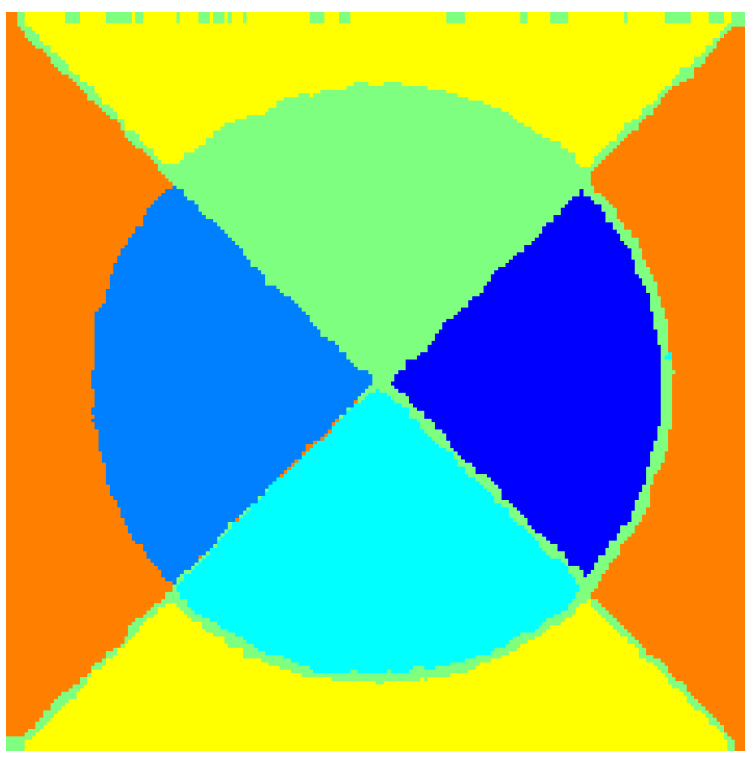

Figura 4.4: Clasificación Wishart. 


\subsubsection{Desempeño}

Un análisis cualitativo de las Figs. 4.3(b) y 4.4 muestra que los dos métodos identifican exitosamente la estructura de la imagen original. En los resultados del algoritmo Wishart, los píxeles mal clasificados se localizan cerca de los bordes de las clases donde la resolución fue degradada por el procesamiento de multiples observaciones. Por otro lado, los bordes son bien preservados por el algoritmo propuesto, pero presenta un etiquetado más ruidoso en las zonas cinco y seis.

Se calculó la matriz de confusión y el estadístico kappa para ambas clasificaciones en base al terreno original simulado de la Fig. 4.1(a) como terreno verdadero. Las Tablas 4.2 y 4.3 muestran las matrices resultantes y la Tabla 4.4 muestra los índices de desempeño correspondientes.

Tabla 4.2: Matriz de confusión para el algoritmo propuesto.

\begin{tabular}{|c|c|c|c|c|c|c|c|}
\hline & \multicolumn{5}{|c|}{ Algoritmo Propuesto } & \multirow[b]{2}{*}{ z6 } \\
\hline & & z1 & z2 & z3 & $\mathrm{z} 4$ & z5 & \\
\hline \multirow{6}{*}{$\begin{array}{l}-0 \\
0 \\
0 \\
> \\
> \\
0 \\
0 \\
0 \\
0 \\
0=0 \\
0 \\
E-1\end{array}$} & Z1 & 10040 & 0 & 1 & 9 & 0 & $\overline{0}$ \\
\hline & $\mathrm{z} 2$ & 0 & 9754 & 1 & 0 & 0 & 12 \\
\hline & z3 & 82 & 438 & 4759 & 113 & 14 & 56 \\
\hline & $\mathrm{z} 4$ & 20 & 130 & 0 & 4799 & 1 & 77 \\
\hline & $\mathrm{z} 5$ & 57 & 18 & 0 & 10 & 4783 & 16 \\
\hline & z6 & 0 & 0 & 0 & 0 & 0 & 4810 \\
\hline
\end{tabular}

Tabla 4.3: Matriz de confusión para el clasificador Wishart

\begin{tabular}{|c|c|c|c|c|c|c|c|}
\hline & \multicolumn{5}{|c|}{ Clasificador Wishart } & \multirow[b]{2}{*}{ z6 } \\
\hline & & $\overline{\mathrm{z} 1}$ & $\overline{z 2}$ & z3 & $\mathrm{z} 4$ & $\mathrm{z} 5$ & \\
\hline \multirow{6}{*}{ 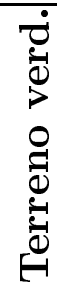 } & $\mathrm{z} 1$ & 9956 & 0 & $\overline{94}$ & 0 & 0 & 0 \\
\hline & $\mathrm{z} 2$ & 6 & 9022 & 738 & 0 & 1 & 0 \\
\hline & $\mathrm{z} 3$ & 61 & 138 & 5217 & 2 & 44 & 0 \\
\hline & $\mathrm{z} 4$ & 158 & 1 & 230 & 4600 & 38 & 0 \\
\hline & $\mathrm{z} 5$ & 24 & 0 & 65 & 0 & 4795 & 0 \\
\hline & $\mathrm{z} 6$ & 0 & 0 & 532 & 0 & 7 & 4271 \\
\hline
\end{tabular}

Las filas en las matrices de confusión indican las clases verdaderas presentes en los datos, mientras las columnas indican las clases identificadas por los algoritmos. 
Tabla 4.4: Índices de desempeño

\begin{tabular}{|c|cc|}
\hline \multirow{2}{*}{ Índice } & \multicolumn{2}{|c|}{ Algoritmo } \\
\cline { 2 - 3 } & Propuesto & Wishart \\
\hline Exactitud general & 0.9736 & 0.9465 \\
Estadístico kappa & $\mathbf{0 . 9 6 7 5}$ & $\mathbf{0 . 9 3 4 3}$ \\
\hline
\end{tabular}

El elemento $(i, j)$ indica el número de píxeles de la clase $i$ que el algoritmo asignó a la clase $j$. Una clasificación perfecta produce una matriz de confusión diagonal. Por lo tanto, la exactitud general se mide con la razón entre la traza y el número total de píxeles.

El índice kappa $\in[0,1]$ mide el nivel de concordancia entre la clasificación lograda con un determinado procedimiento y el terreno verdadero. Este índice no está afectado por el sesgo producido por el tamaño de las clases. Cuanto mayor es el índice, mayor es el nivel de concordancia.

El alto valor de kappa alcanzado por el algoritmo propuesto indica que presenta un nivel de concordancia con el terreno verdadero levemente superior al resultado Wishart.

Estos resultados sugieren que el esquema de clasificación propuesto supera en desempeño al método de Wishart porque obtiene una mejor matriz de confusión y un mejor índice kappa.

\subsection{Desempeño con datos reales}

En esta sección se aplica el algoritmo propuesto a datos SAR SLC reales. Se utiliza un área de $200 \times 200$ píxeles (Fig. 4.5) extraída de un conjunto de datos de $1750 \times 1000$ píxeles de la misión EMISAR (Fig. 4.6), disponible en la página web de la Agencia Espacial Europea (ESA) (European Space Agency, 2014).

\subsubsection{Resultados}

La Fig. 4.7 muestra los valores BIC de la etapa de selección de modelo. El mínimo se alcanza en $K_{\mathrm{EM}}=6$ indicando que se necesitan seis clases para describir los datos. 


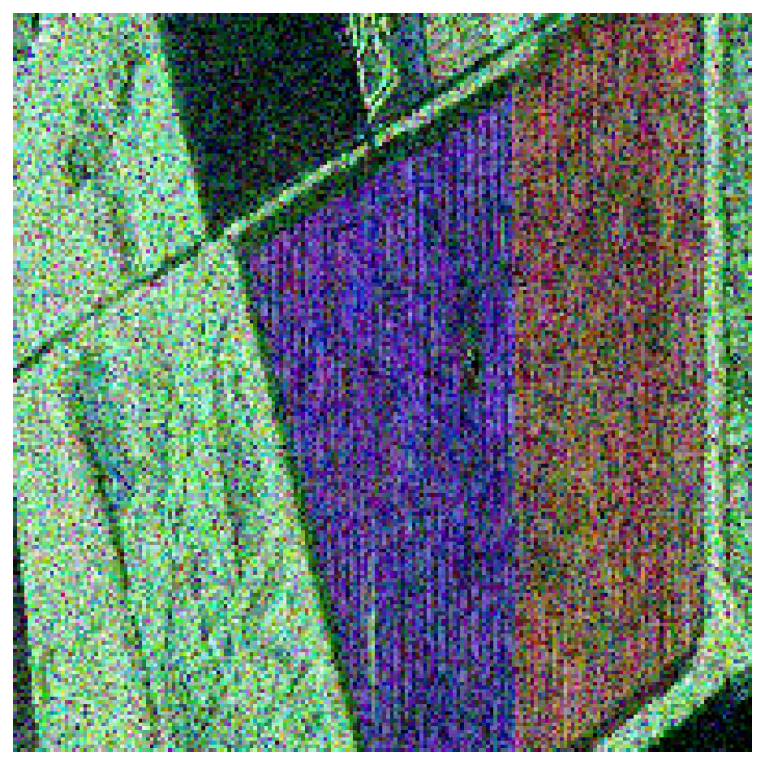

Figura 4.5: Area de interés. Red:|HH-VV|. Green:|HV+VH|. Blue:|HH+VV|.

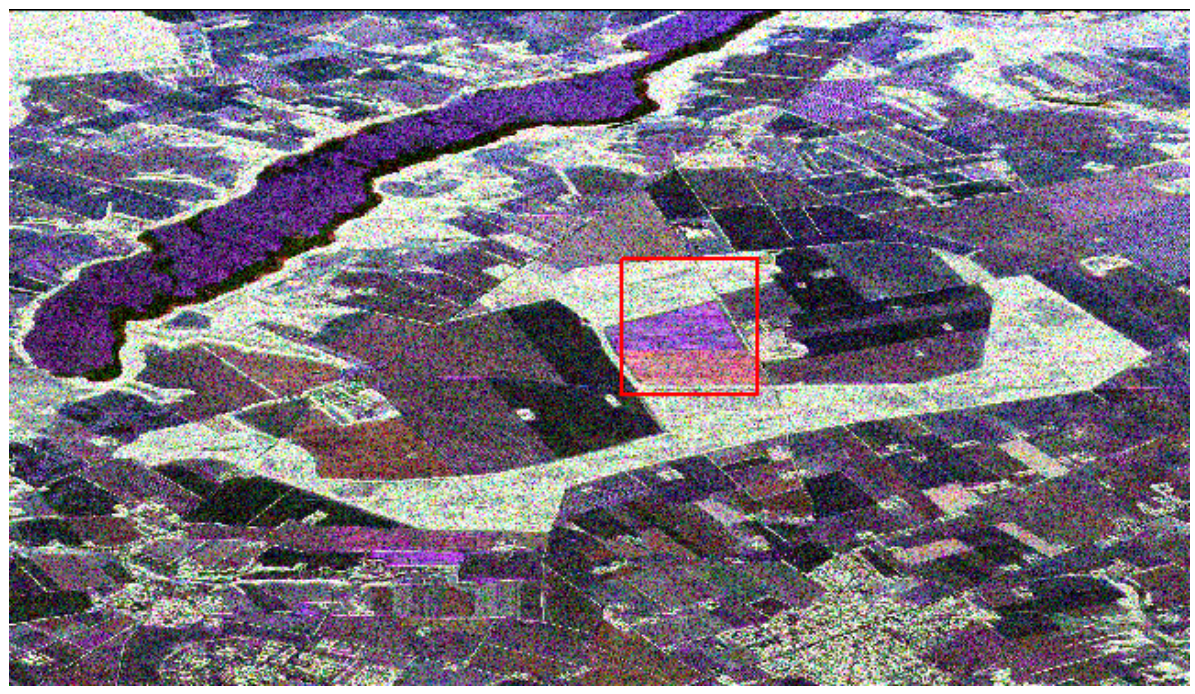

Figura 4.6: Imagen EMISAR completa apaisada. El recuadro rojo indica el área extraída.

La Fig. 4.8(a) muestra los datos etiquetados después de la etapa de refinamiento. El algoritmo CEM reduce aún más el número de clases de $K_{\mathrm{EM}}=6$ a $K_{\mathrm{CEM}}=4$. En la Fig. 4.8(b) se observa el etiquetado final luego del suavizado. EL algoritmo Wishart también fue aplicado al mismo conjunto de datos con cuatro clases inicializado 
aleatoriamente, con un promediado de $5 \times 5$ píxeles. Los resultados se muestran en la Fig. 4.9 .

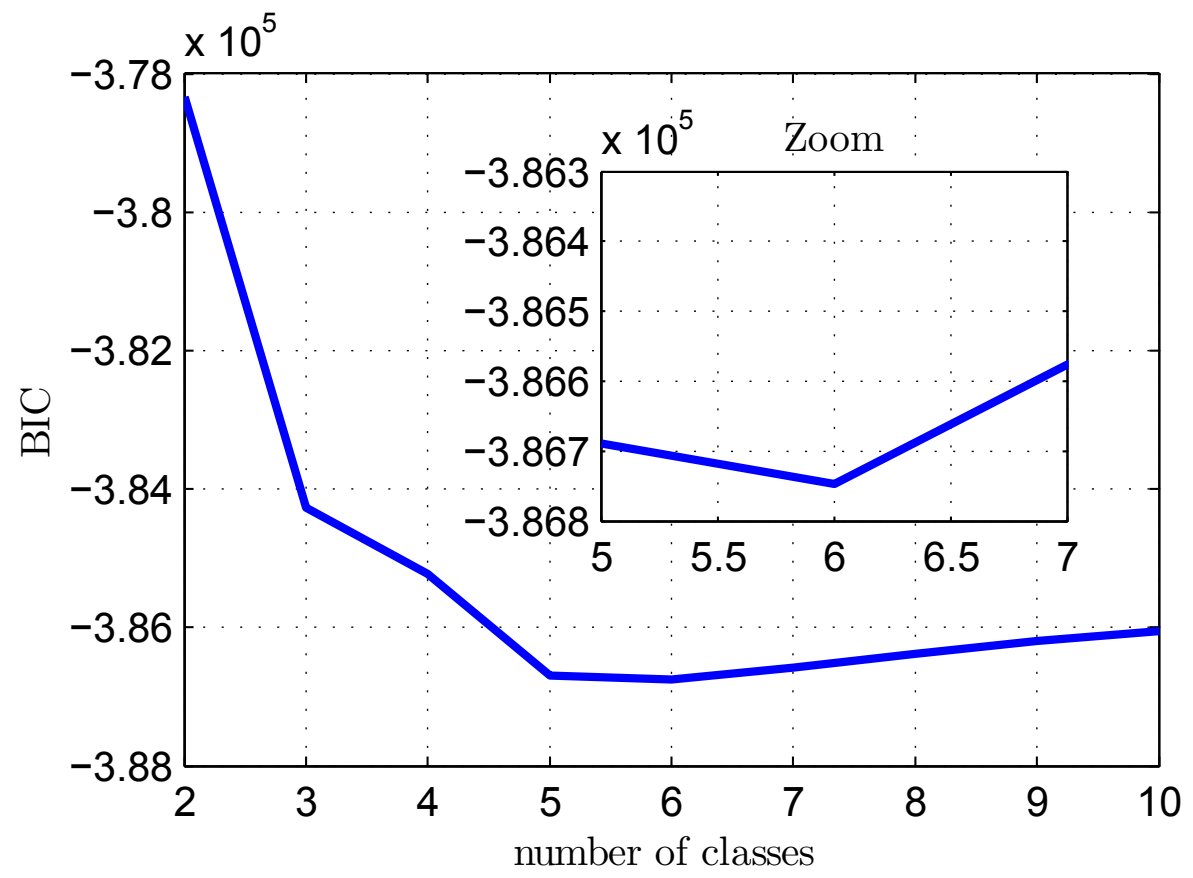

Figura 4.7: Valor BIC para datos reales.

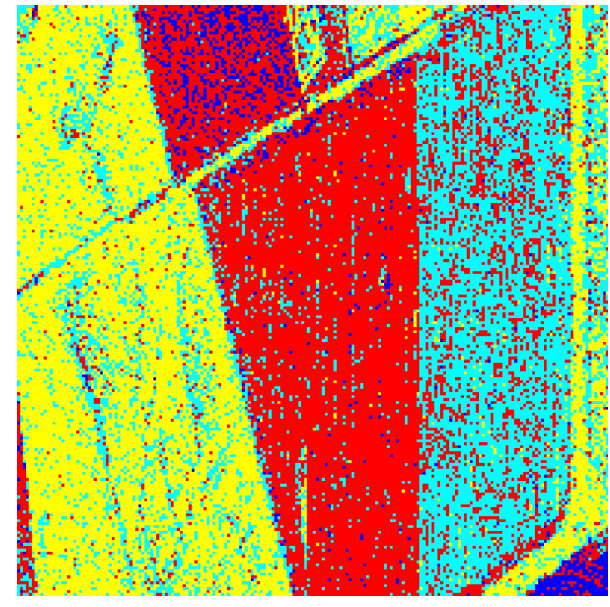

(a)

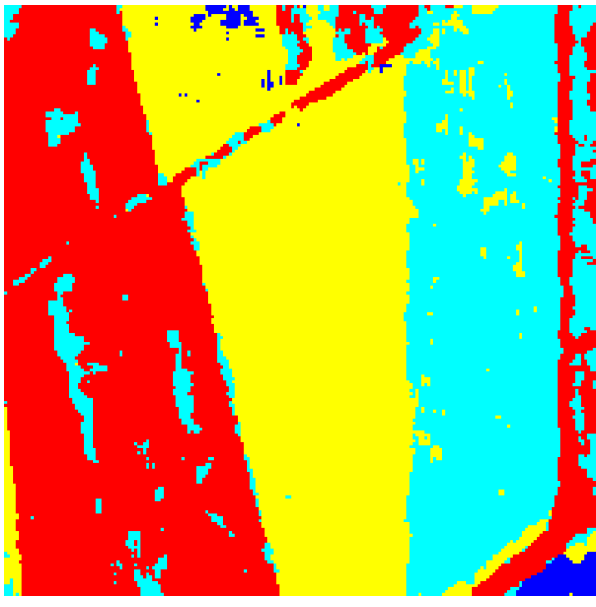

(b)

Figura 4.8: Resultados para datos reales. (a) Refinamiento, (b) Suavizado. 


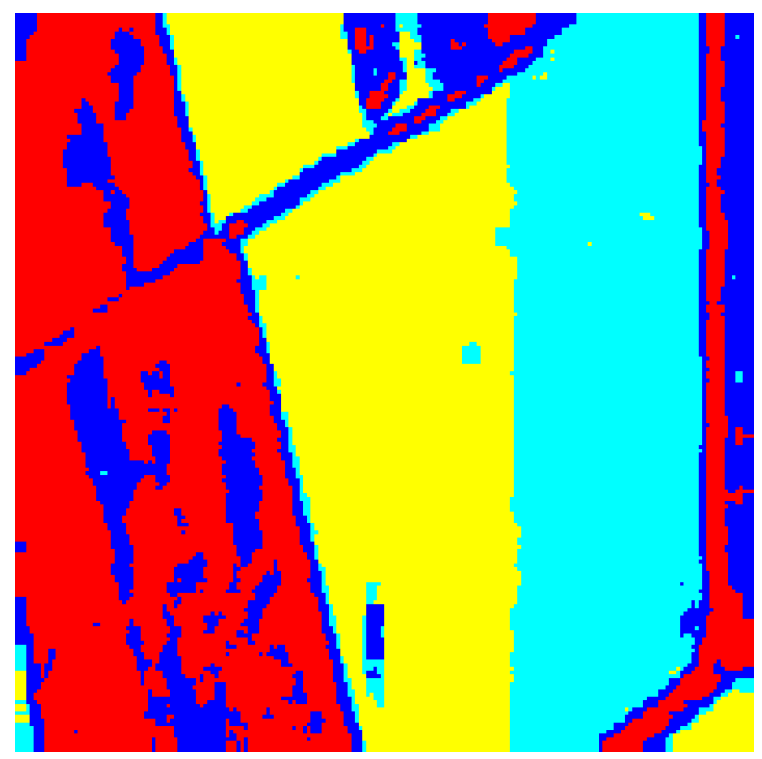

Figura 4.9: Clasificación Wishart para datos reales.

\subsubsection{Desempeño}

En el caso de datos reales, no es posible evaluar el desempeño de los algoritmos con la matriz de confusión debido a que no se dispone del terreno verdadero. Por lo tanto, debe utilizarse una medida de similitud basada en los datos originales y en la imagen etiquetada únicamente. Respecto de este punto, en Davies y Bouldin (1979) los autores definen una medida de similitud promedio $\bar{r}$ de una imagen segmentada que puede utilizarse para dicho propósito.

Denotando con $s_{i}$ la dispersión de la clase $i$ y con $m_{i j}$ la distancia entre centros de las clases $i$ y $j$, la razón $r_{i j}=\left(s_{i}+s_{j}\right) / m_{i j}$ es una medida de similitud estadística entre las clases $i$ y $j$. La similitud promedio se define como:

$$
\bar{r}=\frac{1}{K} \sum_{i=1}^{K} \operatorname{máx}_{j \neq i} r_{i j} .
$$

Cuanto menor es la similitud promedio $\bar{r}$, mayor es la separación entre las clases. Dadas dos clasificaciones $A$ y $B$ con índices $\bar{r}_{A}$ y $\bar{r}_{B}$ respectivamente, se establece que $A$ es mejor que $B$ de acuerdo a este índice si $\bar{r}_{A}<\bar{r}_{B}$. 
Las definiciones de $s_{i}$ y $m_{i j}$ dependen de cada aplicación en particular. En el presente trabajo se utilizan las siguientes definiciones:

$$
\begin{aligned}
\mathbf{m}_{i} & =\frac{1}{n_{i}} \sum_{\boldsymbol{k} \in \text { class } i} \boldsymbol{k}_{i}, \\
s_{i} & =\sqrt{\frac{1}{n_{i}} \sum_{\boldsymbol{k} \in \text { class } i}\left(\boldsymbol{k}_{i}-\mathbf{m}_{i}\right)^{H}\left(\boldsymbol{k}_{i}-\mathbf{m}_{i}\right)}, \\
\operatorname{Cov}_{i} & =\frac{1}{n_{i}} \sum_{\boldsymbol{k} \in \text { clase } i}\left(\boldsymbol{k}_{i}-\mathbf{m}_{i}\right)\left(\boldsymbol{k}_{i}-\mathbf{m}_{i}\right)^{H}, \\
m_{i j} & =\frac{\left\|\operatorname{Cov}_{i}-\operatorname{Cov}_{j}\right\|_{F}}{\left\|\operatorname{Cov}_{i}\right\|_{F}\left\|\operatorname{Cov}_{j}\right\|_{F}},
\end{aligned}
$$

donde $n_{i}$ es el número de elementos en la clase $i,\|\cdot\|_{F}$ representa la norma de Frobenius y $m_{i j}$ es la distancia normalizada entre matrices de covarianza de los datos en las clases $i$ y $j$.

El índice $\bar{r}$ se calculó para el método propuesto y el Wishart, para $K=K_{C E M}$ clases. La Tabla 4.5 muestra los resultados de $\bar{r}$, el valor máximo de dispersión $s_{\max }$ y la mínima distancia entre clases $m_{\min }$ para cada clasificación.

Tabla 4.5: Medida de similitud

\begin{tabular}{|c|cc|}
\hline \multirow{2}{*}{ Índice } & \multicolumn{2}{|c|}{ Algoritmo } \\
\cline { 2 - 3 } & Propuesto & Wishart \\
\hline$s_{\max }$ & 0.3302 & 0.3655 \\
$m_{\min }$ & 0.5728 & 0.5898 \\
$\bar{r}$ & $\mathbf{0 . 2 4 3 0}$ & $\mathbf{0 . 3 8 0 1}$ \\
\hline
\end{tabular}

\subsection{Discusión}

El algoritmo propuesto resuelve exitosamente la clasificación de imágenes SAR polarimétricas donde el modelo complejo gaussiano aplica, es decir, para objetivos homogéneos. 
Los resultados obtenidos para datos simulados y reales muestran que su desempeño es mejor que el correspondiente al algoritmo Wishart en términos de calidad de clasificación. Sin embargo, la comparación se realizó principalmente para corroborar la validez del algoritmo propuesto.

Vale la pena notar que se ha probado el desempeño de la clasificación por comparación con el algoritmo de Wishart para un número fijo de clases $K_{\mathrm{CEM}}$.

Respecto de la habilidad de identificar el número apropiado de clases con datos reales, se ha implementado el proceso de fusión sugerido Cloude y Pottier (1997) para el algoritmo Wishart, y la optimización BIC como en el algoritmo propuesto. Sin embargo, se notó que el número óptimo de clases estimado por cada algoritmo no concordaba en todas los conjuntos de datos probados, por lo que no fue posible realizar una comparación justa y confiable. Debido a que no se dispone de información de terreno verdadero para datos reales, no es posible probar el algoritmo presentado rigurosamente en ese sentido. Alternativamente, se procedió de la siguiente forma. El algoritmo se aplicó sobre tres conjuntos de datos SLC RADARSAT-2 ${ }^{2}$, donde las clases presentes son visualmente distinguibles. Se definió en cada una, una zona de control para cada clase presente, bajo la suposición de que en cada zona sólo hay datos de la clase representada. Esto permite definir un terreno verdadero compuesto por dichas zonas de control y evaluar el desempeño con la matriz de confusión y los índices asociados.

La Fig. 4.10 muestra los resultados. Las imágenes de la izquierda muestran la escena de cada conjunto, con la definición de las zonas de control en cada caso. A la izquierda, se muestra el resultado de cada clasificación. Se observa que en todos los casos, el algoritmo fue capaz de identificar el número correcto de clases y la estructura de la escena.

En las Tablas 4.6 a 4.8 figuran los resultados de la evaluación de desempeño, mostrando una alta concordancia entre el terreno verdadero y la clasificación conseguida, en los tres casos. Estas pruebas manifiestan la habilidad del algoritmo propuesto de identificar la cantidad correcta de clases en una escena.

\footnotetext{
${ }^{2}$ Disponibles en http://gs.mdacorporation.com/SatelliteData/Radarsat2/SampleDataset.aspx
} 
Tabla 4.6: Desempeño para imagen de San Francisco

\begin{tabular}{|c|c|ccc|c|c|}
\cline { 2 - 7 } \multicolumn{2}{c|}{} & \multicolumn{3}{c|}{ Clasificación } & \multicolumn{2}{c|}{ Índices } \\
\cline { 2 - 7 } \multicolumn{2}{c|}{} & edificado & vegetacion & mar & exactitud & kappa \\
\hline$\dot{\vdots}=\bar{\vdots}$ & edificado & $\mathbf{1 6 5 3}$ & 1319 & 15 & & \\
vegetación & 0 & $\mathbf{2 8 5 7}$ & 143 & 0.8565 & 0.7803 \\
& mar & 0 & 2 & $\mathbf{4 3 9 8}$ & & \\
\hline
\end{tabular}

Tabla 4.7: Desempeño para imagen del Estrecho de Gibraltar

\begin{tabular}{|c|c|c|c|c|c|}
\hline & \multicolumn{2}{|c|}{ Clasificación } & \multicolumn{2}{|c|}{ Índices } \\
\hline & & vegetacion & mar & exactitud & kappa \\
\hline$\stackrel{\dot{0}}{\dot{\theta}}$ & $\begin{array}{c}\text { vegetación } \\
\text { mar }\end{array}$ & $\begin{array}{c}39005 \\
1\end{array}$ & $\begin{array}{c}102 \\
\mathbf{3 9 2 6 9}\end{array}$ & 0.9966 & 0.9932 \\
\hline
\end{tabular}

El algoritmo presentado resulta adecuado para aplicaciones donde es necesario acceder a información embebida en el momento de segundo orden de los datos. Por ejemplo, podría utilizarse para identificación de objetivos con estructura conocida. Respecto de este punto, en Hochwald y Nehorai (1995) se presenta una relación entre polarización y la matriz de covarianza, relacionando la matriz con un tipo de objetivo. El costo computacional para determinar el orden de modelo lleva a expresar los datos polarimétricos con un CGMM de mínima complejidad, sin utilizar información previa. Esto sugiere que el método propuesto podría utilizarse como inicialización de otros algoritmos de clasificación.

\subsection{Conclusiones}

Se ha presentado el desarrollo de un algoritmo de clasificación no supervisado para datos SAR SLC, basado en un modelo CGMM. El algoritmo fue aplicado exitosamente para datos SAR simulados y reales. Los resultados fueron comparados con aquellos obtenidos por el algoritmo Wishart por medio la matriz de confusión y el estadístico kappa para datos simulados, y por medio de una medida de dispersión para datos reales. El algoritmo propuesto se desempeñó mejor en términos de esos índices. A pesar de que no se utilizó filtrado de speckle ni información de píxeles vecinos, produjo clasificaciones correctas sin utilizar información previa como el 
Tabla 4.8: Desempeño para imagen de Flevoland

\begin{tabular}{|c|c|c|c|c|c|c|}
\hline & \multicolumn{3}{|c|}{ Clasificación } & \multicolumn{2}{|c|}{ İndices } \\
\hline & & bosque & sembradc & pasto & exactitud & kappa \\
\hline \multirow{3}{*}{$E$} & bosque & 1999 & 1 & 0 & & \\
\hline & sembrado & 0 & 750 & 0 & 0.9936 & 0.9887 \\
\hline & pasto & 20 & 1 & 654 & & \\
\hline
\end{tabular}

número de clases o zonas de entrenamiento. Además, se ha probado el algoritmo propuesto en varias imágenes, no mostradas en este trabajo. Los índices calculados resultaron similares o mejores que los correspondientes al algoritmo Wishart en siete de diez casos, lo que parece favorecer a al método propuesto.

En el siguiente capítulo se abordará la clasificación de datos no homogéneos, donde el modelo gaussiano debe abandonarse para considerar la textura. Sin embargo, se mantendrá el enfoque del modelo de mezclas de densidades y el método EM para el desarrollo de un clasificador no supervisado. 

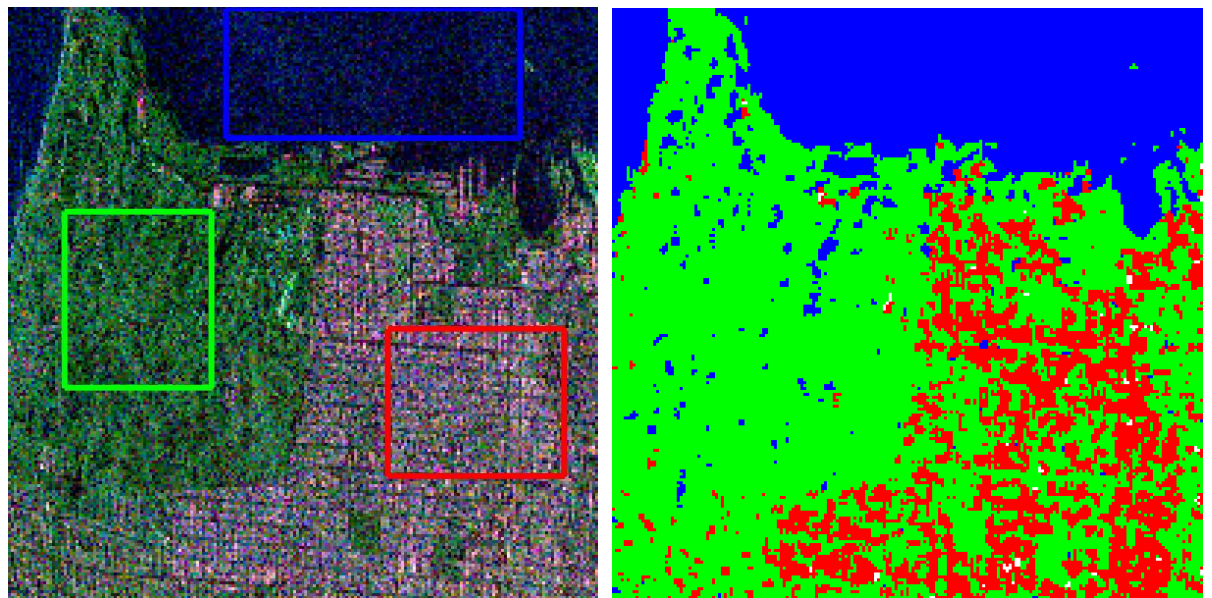

(a) San Francisco. Zonas de interés: edificado, vegetación, mar.
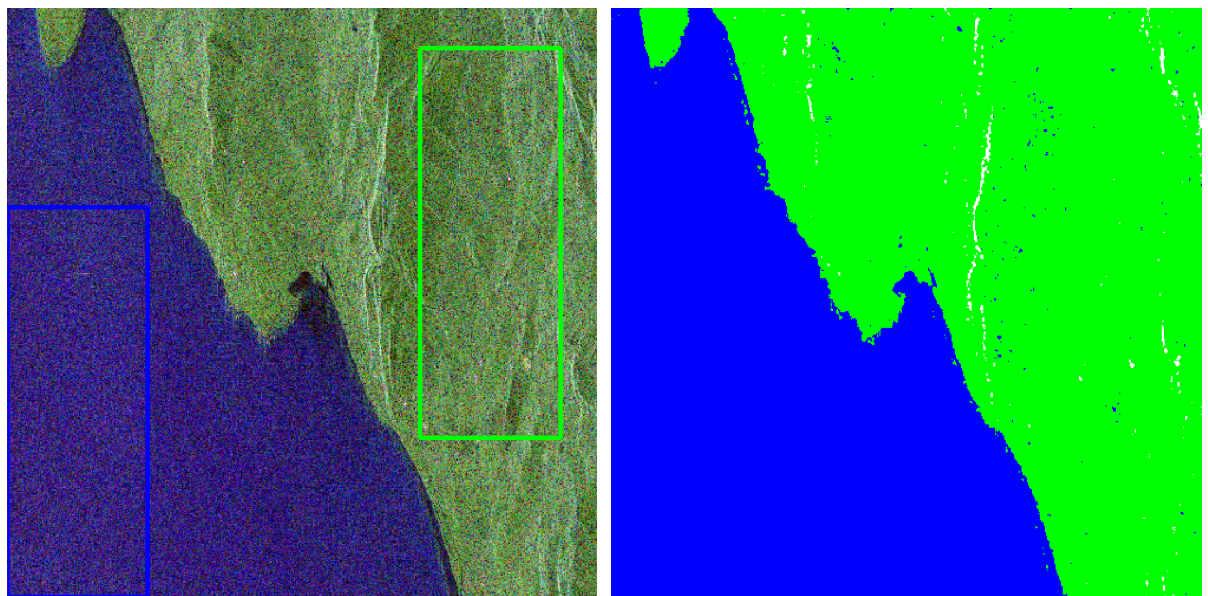

(b) Estrecho de Gibraltar. Zonas de interés: vegetación, mar.
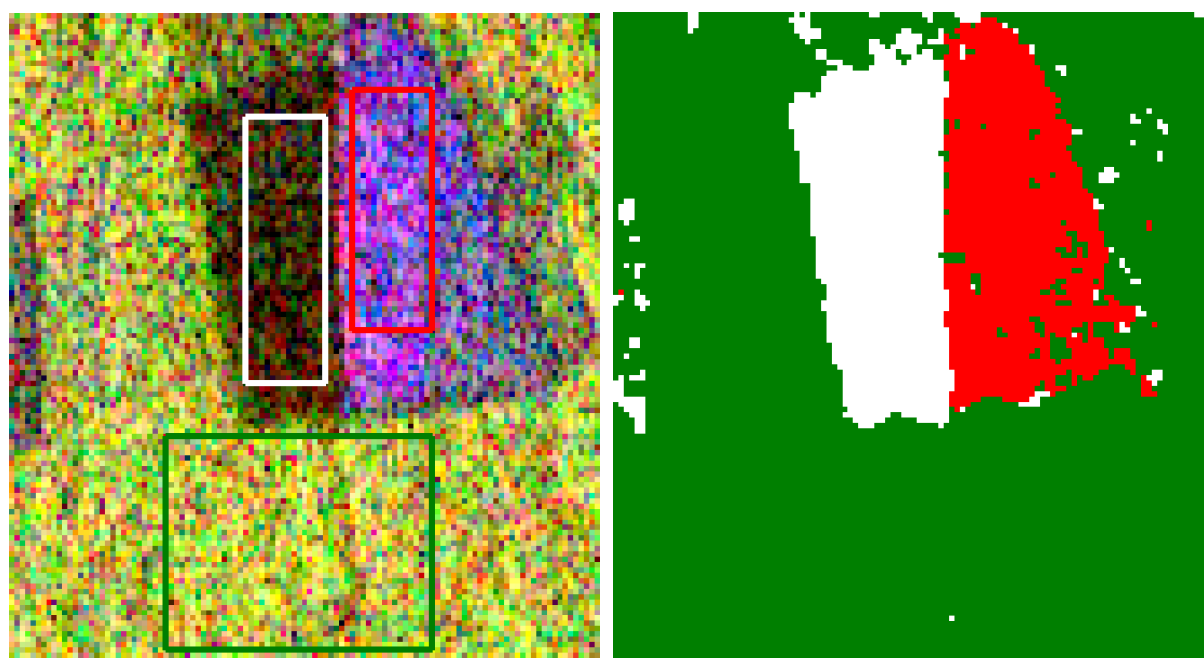

(c) Flevoland. Zonas de interés: bosque, sembrado, pasto

Figura 4.10: Imágenes RADARSAT-2. Izquierda: zonas de interés. Derecha: clasificación. 


\section{Capítulo 5}

\section{Clasificación para datos no-homogéneos}

\subsection{Introducción}

La clásica densidad de Wishart basada en el modelo complejo gausssiano de los coeficientes de dispersión describe correctamente areas homogéneas sin textura con speckle completamente desarrollado. El conocido método de clasificación no supervisado presentando en Lee et al. (1999) está basado en la mencionada distribución y usa la descomposición $H / \alpha$ Cloude y Pottier (1997) como inicialización. Sin embargo, la creciente resolución alcanzada por los actuales SAR debido a la continua mejora de la tecnología provoca que los modelos clásicos no siempre se ajusten a los datos medidos. Por lo tanto, surge la necesidad de utilizar modelos más complejos para interpretar las observaciones.

A este respecto, en Frery et al. (1997) los autores han desarrollado la familia de distribuciones $\mathcal{G}$, capaz de describir datos altamente heterogéneos. Sin embargo, la versatilidad del modelo $\mathcal{G}$ tiene como contraparte la dificultad en la estimación de sus parámetros. En Horta et al. (2008b) los autores proponen una mezcla de densidades $\mathcal{G}_{p}^{0}$ y el método SEM para clasificar datos SAR polarimétricos, utilizando el método de los momentos para estimar sus parámetros. En Horta et al. (2008a) su trabajo es extendido con el estudio de diferentes inicializaciones.

En este capítulo se propone la utilización del método EM para la clasificación de datos SAR polarimétricos modelados con mezcla de densidades $\mathcal{G}_{p}^{0}$. Se trata de 
un método iterativo no supervisado donde sólo se informa al algoritmo el ENL de la imagen. No requiere ninguna información adicional para su inicialización. Debido a que se utiliza el mismo modelo de datos y un enfoque basado en EM como en Horta et al. (2008b), el presente algoritmo puede considerarse como su extensión no supervisada que provee los estimados de máxima verosimilitud de las matrices de covarianza y de los parámetros de forma y escala.

\subsection{Modelo de Datos}

Como se vio en apartados anteriores, los datos SAR se construyen a partir de la señal de retorno que está relacionada con las propiedades dieléctricas de la superficie. Los datos polarimétricos del SAR monoestático se forman mediante el vector de objetivo $\boldsymbol{k}=\left[\begin{array}{lll}s_{h h} & \sqrt{2} s_{h v} & s_{v v}\end{array}\right]$. En áreas homogéneas el vector de objetivo $\boldsymbol{k}$ se describe por la densidad compleja gaussiana. Los datos asociados de múltiples observaciones MLC, definidos como $Z=\sum_{i=1}^{n} \boldsymbol{k}_{i} \boldsymbol{k}_{i}^{H} / n$ siguen una distribución Wishart compleja $\mathcal{W C}(C, n)$, donde $C=\mathcal{E}\left\{\boldsymbol{k} \boldsymbol{k}^{H}\right\}$ es la matriz de covarianza, $n$ es el número de observaciones (looks), $(\cdot)^{H}$ denota transposición y conjugación, y $\mathcal{E}\{\cdot\}$ indica la operación esperanza.

En áreas no homogéneas el vector de objetivo se desvía de la estadística gaussiana. Cada dato polarimétrico $Z$ puede ser descripto como el producto de dos variables aleatorias $Z=\mathbf{X} Y$, donde $\mathbf{X} \in \mathbb{R}^{+}$describe la dispersión del terreno e $Y \in \mathbb{C}^{3 \times 3}$ contiene la información polarimétrica y el speckle. Para describir los datos MLC, se utilizará la densidad $\mathcal{G}_{p}^{0}$ resultante (ver cap.3), que tiene la siguiente expresión:

$$
f(Z ; \boldsymbol{\theta})=\frac{n^{n d}|Z|^{n-d} \Gamma(d n-\alpha)}{h(n, d)|C|^{n} \Gamma(-\alpha) \gamma^{\alpha}}\left(n \operatorname{tr}\left(C^{-1} Z\right)+\gamma\right)^{\alpha-d n},
$$

donde $\boldsymbol{\theta}=(\alpha, \gamma, C, n), h(n, d)=\pi^{d(d-1) / 2} \Gamma(n) \ldots \Gamma(n-d+1)$ and $d=3$ es el número de polarizaciones. El parámetro $\alpha<0$ está directamente relacionado con la rugosidad del terreno y $C$ describe sus características polarimétricas. Los símbolos $\operatorname{tr}(\cdot)$ y $|$.$| indican la traza y el determinante respectivamente.$ 


\subsubsection{Modelo de mezclas}

Los datos se describen por un modelo de mezcla de $K$ clases dado por:

$$
f(Z, \boldsymbol{\Theta})=\sum_{j=1}^{K} \omega_{j} f_{j}\left(Z ; \boldsymbol{\theta}_{j}\right)
$$

donde $f_{1}, \ldots, f_{K}$ son las $K$ densidades de probabilidad dadas en (5.1). Cada clase se describe con un conjunto de parámetros $\boldsymbol{\theta}_{j}=\left(\alpha_{j}, \gamma_{j}, C_{j}, n_{j}\right)$. Los coeficientes $w_{j}$ indican la proporción de la $j$-ésima componente en la mezcla, sujetas a las restricciones $\sum_{j=1}^{K} \omega_{j}=1$ y $\omega_{j} \geq 0, j=1 \ldots K$. El vector de parámetros de la mezcla es $\boldsymbol{\Theta}=\left(\omega_{1}, \ldots, \omega_{K}, \boldsymbol{\theta}_{1}, \ldots, \boldsymbol{\theta}_{K}\right)$.

\subsection{Estructura del algoritmo}

El algoritmo propuesto sigue una estrategia descendente (top-down). Comienza con una única clase y divide el conjunto de datos iterativamente en más clases conforme encuentra evidencia de estructuras más complejas dentro de ellas. Esta etapa provee una inicialización adecuada al algoritmo EM, que estima el vector de parámetros de la mezcla y la imagen etiquetada. Finalmente, un filtro no lineal de moda se aplica para suavizar el resultado, como en el algoritmo de clasificación para datos homogéneos.

Luego de una inicialización trivial, prosigue la etapa de división-fusión que determina la cantidad de clases en el conjunto de datos. Se basa en el test de hipótesis para las matrices de covarianza de distribución Wishart propuesto en Conradsen et al. (2003) y en el clasificador basado en la misma distribución (Lee et al., 1999). Esta etapa provee el número de clases de la mezcla y la inicialización (imagen etiquetada) a la siguiente etapa. El paso de estimación aplica el algoritmo EM al modelo de mezclas de densidades $\mathcal{G}_{p}^{0}$ para estimar su vector de parámetros $\boldsymbol{\Theta}=\left(\omega_{1}, \ldots, \omega_{K}, \boldsymbol{\theta}_{1}, \ldots, \boldsymbol{\theta}_{K}\right)$. En la siguiente etapa se realiza la clasificación MAP de cada pixel en el conjunto de datos en base a los parámetros estimados. Final- 
mente, se aplica el filtrado no lineal para suvizar el resultado. El pseudocódigo del procedimiento se presenta en Algoritmo 2.

\subsubsection{Inicialización}

La inicialización del algoritmo es muy sencilla, y consiste en asignar todos los píxeles (datos) a una única clase. Este es el primer paso de la estrategia descendente para capturar la estructura de clases.

\subsubsection{División-fusión}

En esta etapa se obtienen el número de clases $K$ presentes en los datos, una partición de los datos $P_{K}$ y un conjunto de matrices de covarianza $\{M\}=\left\{M_{1}, \ldots, M_{K}\right\}$, que describen cada centro de clase. La partición $P_{K}$ y el conjunto de $\{M\}=$ $\left\{M_{1}, \ldots, M_{K}\right\}$ serán utilizados como inicialización por la siguiente etapa. Debe notarse que si bien las $M_{j}$ son matrices de covarianza, no son denotadas con $C_{j}$ pues no son las matrices estimadas del modelo 5.2. Las matrices $M_{j}$ sólo actúan como descriptores de clase en esta instancia.

Esta etapa procede iterativamente. Suponiendo que hay $K_{L}$ clases y una partición $P_{K_{L}}$ en la iteración $L$-ésima, el clasificador Wishart se aplica a los datos de a cada clase por separado para identificar dos subclases. Luego de alcanzar la convergencia, se tienen dos matrices $M_{i 1}$ and $M_{i 2}$ como los centros estimados de las subclases, para cada clase $\mathcal{C}_{i}$. Luego debe determinarse si estas dos subclases están lo suficientemente separadas estadísticamente como para considerarlas dos clases completamente diferentes. Para eso, se utiliza el test estadístico para matrices Wishart desarrollado en Conradsen et al. (2003) como una medida de separación entre clases.

Se considera como hipótesis nula $H_{0}: M_{i 1}=M_{i 2}$ contra la alternativa $H_{1}$ : $M_{i 1} \neq M_{i 2}$. Bajo $H_{0}$ el cociente de verosimilitud resulta

$$
\log Q=n\left(2 d \log 2+\log \left|M_{i 1}\right|+\log \left|M_{i 2}\right|-2 \log \left|M_{i 1}+M_{i 2}\right|\right)
$$


donde $n$ es el número equivalente de observaciones. La probabilidad de encontrar un valor $z$ menor a $-2 \rho \log Q$ is

$$
\begin{aligned}
P\{-2 \rho \log Q \leq z\}= & P\left\{\chi^{2}\left(d^{2}\right) \leq z\right\}+w P\left\{\chi^{2}\left(d^{2}+4\right) \leq z\right\}- \\
& w P\left\{\chi^{2}\left(d^{2}\right) \leq z\right\}
\end{aligned}
$$

donde $\rho=1-17 / 12 n$ y $w=-d^{2}(1-1 / \rho)^{2} / 4+7 d^{2}\left(d^{2}-1\right) /\left(96 n^{2} \rho^{2}\right)$.

Dada una probabilidad de falsa alarma (PFA), se calcula el umbral $\Lambda$ tal que

$$
P\{-2 \rho \log Q \leq \Lambda\}=1-\mathrm{PFA}
$$

utilizando (5.4) y se compara con $Q^{\prime}=-2 \rho \log Q$. Si $Q^{\prime} \leq \Lambda$, se acepta $H_{0}$ y la clase no es dividida. Si $Q^{\prime}>\Lambda, H_{0}$ se rechaza, ya que existe suficiente evidencia para suponer que las matrices involucradas describen diferentes clases. Por lo tanto, la clase se divide de acuerdo a la clasificación Wishart y las matrices $M_{i 1}$ y $M_{i 2}$ son los nuevos centros de clase.

Luego de aplicar este proceso de división a las $K_{L}$ clases, es necesario aplicar una etapa de fusión porque las subclases pertenecientes a diferentes clases no fueron evaluadas, y es posible que pertenezcan a la misma clase. Se aplica entonces (5.3) para todas las matrices que resultaron del proceso de división: $M_{i k}, M_{j r}$; $i, j=1 \ldots K_{L}, i \neq j ; k, r=\{1,2\}$. Utilizando la misma PFA, aquellos test cuyos valores resulten más pequeños que $\Lambda$, indican aceptación de $H_{0}$, es decir, pertenecen a la misma clase y son candidatos a ser fusionados. Se toma el más pequeño de esos valores, correspondientes a las matrices más similares, y se fusionan las clases involucradas: se re-etiquetan los píxeles con un único valor y se calcula un nuevo centro de clase igual la media entre los centros de las clases fusionadas.

Luego de este proceso de división-fusión, resultan $K_{L+1}$ clases, con $K_{L+1} \in$ $\left[K_{L}, 2 K_{L}\right]$. En la siguiente iteración $L+1$, el proceso se repite para $K_{L+1}$ clases. La etapa concluye cuando $K_{M}=K_{M+1}=K$ y no hubo clases fusionadas en la iteración $M$-ésima.

Este simple procedimiento permite identificar la estructura de clases del conjunto de datos utilizando el número de observaciones como la única información requerida. 
El PFA actúa como variable de ajuste, estableciendo la sensibilidad del proceso para diferenciar clases.

\subsubsection{Estimación}

Para estimar el vector de parámetros $\Theta$ del modelo de mezclas (5.2), se aplica el algoritmo EM con $K$ clases, partición $P_{K}$ y centro de clases $\{M\}$ como inicialización. Los detalles del cálculo se encuentran en el Apéndice 5.A. Las expresiones resultantes para el l-ésimo paso son:

Paso E:

$$
\gamma_{i j}^{(l)}=\frac{\omega_{j}^{(l-1)} f_{j}\left(Z_{i}, \boldsymbol{\theta}_{j}^{(l-1)}\right)}{\sum_{r=1}^{K} \omega_{r}^{(l-1)} f_{r}\left(Z_{i}, \boldsymbol{\theta}_{r}^{(l-1)}\right)} .
$$

Paso M:

$$
\begin{gathered}
\omega_{j}^{(l)}=\frac{\sum_{i=1}^{N} \gamma_{i j}^{(l)}}{N}=\frac{N_{j}^{(l)}}{N}, \\
C_{j}^{(l)}=-\nu_{j} \sum_{i=1}^{N} \gamma_{i j}^{(l)} \frac{Z_{i}}{n \operatorname{tr}\left(C_{j}^{(l)}{ }^{-1} Z_{i}\right)+\gamma_{j}}, \\
\mathcal{L}\left(\alpha_{j}, \gamma_{j}, C_{j}\right)=N_{j}^{(l)} \log \left(\Gamma\left(n d-\alpha_{j}\right)\right)-N_{j}^{(l)} \log \left(\Gamma\left(-\alpha_{j}\right)\right)-N_{j}^{(l)} \alpha_{j} \log \left(\gamma_{j}\right) \\
+\sum_{i=1}^{N} \gamma_{i j}^{(l)}\left(\alpha_{j}-n d\right) \log \left(n \operatorname{tr}\left(C_{j}^{-1} Z_{i}\right)+\gamma_{j}\right),
\end{gathered}
$$

donde $\nu_{j}=\left(n d-\alpha_{j}\right) / N_{j}^{(l)} n, N_{j}^{(l)}=\sum_{i} \gamma_{i j}$ y $N$ es número total de píxeles.

La Ec. (5.6) calcula la probabilidad a posteriori de que el dato $i$ pertenezca a la clase $j$, en la iteración $l$, mientras que la Ec. (5.8) calcula la matriz de covarianza de cada clase. $\mathcal{L}\left(\alpha_{j}, \gamma_{j}, C_{j}\right)$ es la expresión a maximizar con respecto a $\alpha_{j}$ y $\gamma_{j}$.

Debido a que las Ecs. (5.8) y (5.9) no son independientes, se utiliza el método EM Condicional (ECM) (McLachlan y Krishnan, 2008) que consiste en maximizar cada variable condicionada a las restantes, es decir, restringiendo el espacio de parámetros en cada maximización de forma que el conjunto de restricciones reproduzca el espacio de parámetros completo. Por lo tanto, primero se resulve (5.8) con $\alpha_{j}$ y $\gamma_{j}$ fijas, 
y luego se maximiza $\mathcal{L}\left(\alpha_{j}, \gamma_{j}, C_{j}\right)$ con la $C_{j}$ obtenida. Ambos procedimientos son realizados numéricamente, para cada iteración del algoritmo EM.

La solución para la expresión (5.8) requiere una explicación mas profunda. Se trata de una ecuación trascendental sin solución analítica. Para resolverla, se utiliza el principio derivado en Conte et al. (2002), con el que se halla $C_{j}^{(l)}$ recursivamente como

$$
C_{j}^{(t+1)}=-\nu_{j} \sum_{i=1}^{N} \gamma_{i j}^{(l)} \frac{Z_{i}}{n \operatorname{tr}\left(C_{j}^{-1^{(t)}} Z_{i}\right)+\gamma_{j}},
$$

inicializando con la matriz de covarianza calculada en la iteración previa de EM: $C_{j}{ }^{(t=0)}=C_{j}{ }^{(l-1)}$. En Conte et al. (2002) se demuestra que para $n=1 \mathrm{y} \gamma_{j}=0$ esta recursión mejora la estimación de la covarianza a medida que $t$ crece en el sentido que la secuencia de estimados de dicha matriz aumenta el valor de la función de verosimilitud, que es precisamente el objetivo de la recursión EM. Adicionalmente, en Pascal et al. (2008) se demuestra su convergencia para cualquier inicialización.

Si bien no se demuestra aquí la convergencia de la recursión propuesta cuando $n \neq 1$ y $\gamma \neq 0$, se probó extensamente en gran variedad de casos y en todos ellos convergió al valor correcto.

La recursión termina cuando

$$
\epsilon=\frac{\left\|C_{j}^{(t+1)}-C_{j}^{(t)}\right\|_{F}}{\left\|C_{j}^{(t)}\right\|_{F}}
$$

se vuelve más pequeño que un valor predefinido. $\|\cdot\|_{F}$ representa la norma de Frobenius. Cuando se alcanza la convergencia, $C_{j}^{(t)} \approx C_{j}^{(t+1)}=C_{j}^{(l)}$.

Luego de que el algoritmo EM converge, se dispone de los estimados $\hat{\Theta}$ que describen completamente el modelo de mezcla (5.2). Debido a que el proceso de estimación completo comprende dos algoritmos iterativos anidados (EM y la estimación de la matriz de covarianza), puede insumir mucho tiempo si se utiliza el conjunto de datos completo. Por lo tanto puede tomarse un subconjunto representativo del mismo para acelerar el proceso. En las pruebas realizadas, se utilizó un subconjunto formado por el muestreo aleatorio con un factor de 0.4 (40\% del conjunto original) sin degradar apreciablemente los resultados de la estimación. 


\subsubsection{Clasificación}

En esta etapa se realiza la clasificación MAP. Cada dato del conjunto original $Z_{i}$ se asigna a la clase con la verosimilitud más alta:

$$
\begin{aligned}
& \mathcal{C}_{j} \leftarrow Z_{i}, j=\underset{j}{\operatorname{argmáx}} f_{j}\left(Z_{i}, \boldsymbol{\theta}_{j}\right), \\
& \forall i \in[1 \ldots N], \forall j \in[1 \ldots K] .
\end{aligned}
$$

\subsubsection{Suavizado}

Análogamente a lo que sucedía en algoritmo presentado en Cap.4, la imagen clasificada puede resultar ruidosa debido a que no se utiliza información de los píxeles vecinos en la clasificación. El mismo filtro no lineal basado en la operación moda propuesto en 4.3.4 se aplica para suavizar la imagen resultante, esta vez utilizando $h=w=3$.

\subsection{Desempeño con datos simulados}

Para evaluar el desempeño del algoritmo propuesto, se utilizó un análisis Montecarlo. Se simularon imágenes SAR compuestas de cuatro clases de acuerdo al modelo (5.2). Cada clase se describe con una densidad $\mathcal{G}_{p}^{0}$ y cada una de ellas contribuye a la mezcla con igual peso $\left(\omega_{j}=1 / 4, j=1 \ldots 4\right)$. Los datos de cada clase fueron generados de acuerdo al procedimiento sugerido en Horta et al. (2008b) utilizando el modelo multiplicativo descripto en la Sec. 5.2 y $n=4$, con los parámetros de la Tabla 5.1,

Tabla 5.1: Parámetros de los datos simulados

\begin{tabular}{|c|c|c|}
\hline Color & $\alpha_{j}$ & $C_{j}$ \\
\hline \hline cyan & $-1,2$ & $\operatorname{Toepl}\left(\left[\begin{array}{lll}1 & \rho_{1} & \rho_{1}^{2}\end{array}\right]\right)$ \\
\hline rojo & $-1,8$ & $\operatorname{Toepl}\left(\left[\begin{array}{lll}1 & \rho_{2} & \rho_{2}^{2}\end{array}\right]\right)$ \\
\hline amarillo & -3 & $\operatorname{Toepl}\left(\left[\begin{array}{lll}1 & \rho_{3} & \rho_{3}^{2}\end{array}\right]\right)$ \\
\hline azul & -10 & $\operatorname{Toepl}\left(\left[\begin{array}{lll}1 & \rho_{4} & \rho_{4}^{2}\end{array}\right]\right)$ \\
\hline
\end{tabular}


\begin{tabular}{l} 
Algoritmo 2 Pseudocódigo del algoritmo propuesto \\
\hline 1: Inicialización: \\
- Etiquetar la imagen completa con una única clase.
\end{tabular}

2: División-fusión: Repetir mientras nuevo número de clases $\neq$ número de clases previo:

- $K \leftarrow$ número de clases previos

- para $\mathrm{k}=1: \mathrm{K}$ (cada clase):

- Aplicar el clasificador Wishart para dos clases e inicialización aleatoria.

- Calcular el test de hipótesis sobre ambas matrices de covarianza estimadas.

- Si se acepta $H_{0}$ : no realizar la división.

- Si se rechaza $H_{0}$ : confirmar la división, re-etiquetar de acuerdo a la clasificación Wishart, $K \leftarrow K+1$.

- fin para

- Calcular el test de hipótesis sobre $M_{l}$ and $M_{m}$, para $l, m \in[1: K], l \neq m$.

- Fusionar clases cuyo test no rechazó $H_{0}$ y tomó el mínimo valor. $K \leftarrow K-1$.

- Nuevo número de clases $\leftarrow K$.

3: Estimación:

- Elegir aleatoriamente un subconjunto de datos $Z_{\text {sub }}$.

- Aplicar EM a $Z_{\text {sub }}$, para el número de clases y el etiquetado inicial resultante de la etapa división-fusión.

- Estimación resultante $\leftarrow \hat{\Theta}$.

4: Clasificación:

- Asignar cada dato original $Z_{i}$ a la clase $j=\operatorname{argmáx}_{j} f_{j}\left(Z_{i}, \boldsymbol{\theta}_{j}\right)$.

5: Suavizado:

- Aplicar filtro de moda. 
donde $\rho_{1}=0,8003+j 0,1419, \rho_{2}=0,4715-j 0,1927, \rho 3=0,1576-j 0,9706 \mathrm{y}$ $\rho 4=-0,4404-j 0,1645$. Estos valores se utilizaron en Formont et al. (2013) para generar matrices SAR polarimétricas. La expresión Toepl $\left(\left[\begin{array}{lll}a & b & c\end{array}\right]\right)$ indica la matriz Hermítica Toeplitz con primera columna $[a b c]$.

En cada configuración Montecarlo se simuló una imagen SAR compuesta de cuatro clases con covarianzas $\mathbf{C}_{j}$ y $\alpha$ y $n$ tomados de las listas $n=\{5,7,9,15,25\}$, $\alpha=\{-1,5,-2,-2,5,-3,-3,5,-4,-4,5,-5,-5,5,-6,-10\}$. Se generaron 50 conjuntos de datos para cada configuración, dando un total de 2750 realizaciones.

La Fig. 5.1(a) muestra el terreno simulado con cuatro zonas de $100 \times 100$ píxeles cada una. La Fig. 5.1(b) la imagen MLC en intensidad (span) de una realización a modo de ejemplo. Cada zona se identifica con una clase del modelo de mezcla simulado.

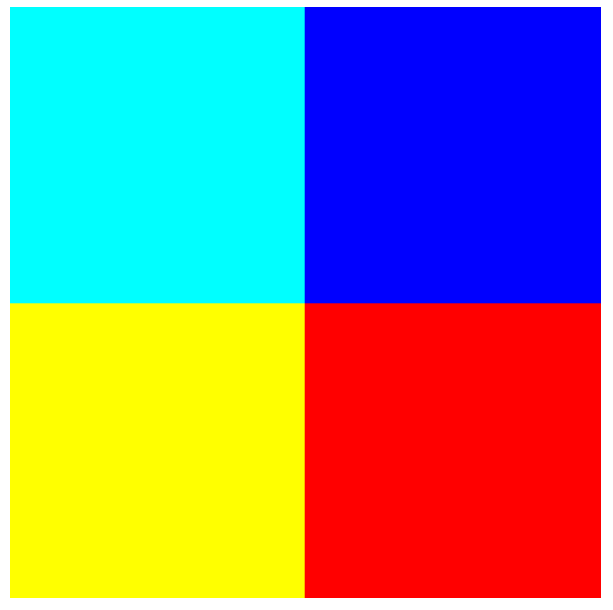

(a)

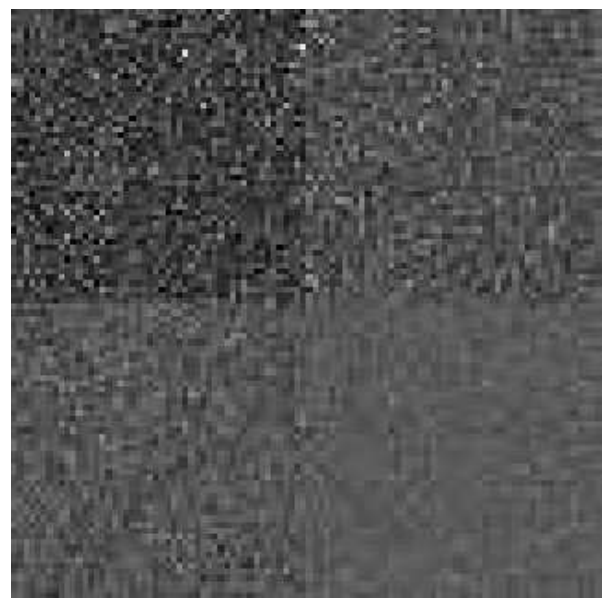

(b)

Figura 5.1: (a): Terreno simulado. (b): Imagen de intensidad (span).

\subsubsection{Resultados}

El algoritmo propuesto se aplicó a cada realización Montecarlo. Se evaluaron dos aspectos diferentes del algoritmo: su habilidad para identificar el número correcto de clases y la exactitud de la clasificación. El primero se evaluó contando la cantidad de clasificaciones resultantes que identificaron las cuatro clases presentes, sobre el 


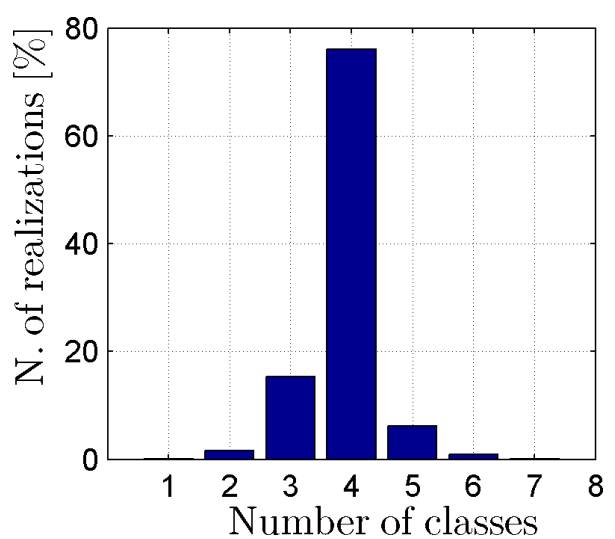

(a)

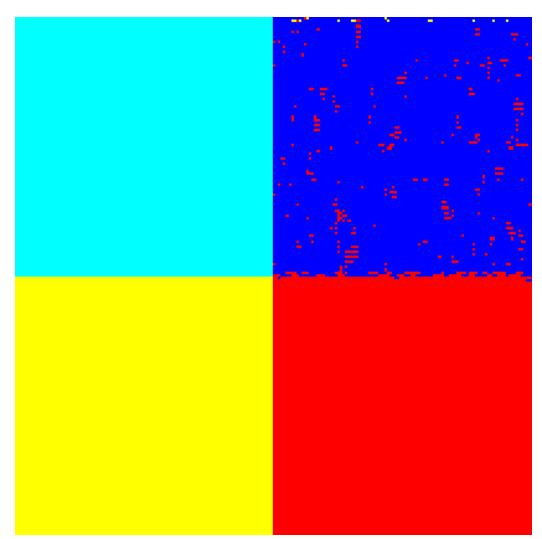

(b)

Figura 5.2: (a): Histograma de la cantidad de clases detectada sobre las 2750 realizaciones. (b): Resultado de clasificación (ejemplo) para datos simulados.

total de clasificaciones. Para evaluar la exactitud se utilizó la matriz de confusión y el índice asociado kappa. El promedio de los valores obtenidos de kappa de todas las clasificaciones es una medida de la exactitud del algoritmo. También se aplicó el algoritmo descripto en Horta et al. (2008b) al mismo conjunto de datos para comparar los desempeños. Dado que se trata de un algoritmo supervisado, se informó el número de clases (cuatro) a identificar.

La Fig. 5.2(a) presenta los resultados del análisis Montecarlo para el número de clases identificado, mostrando baja dispersión alrededor del valor correcto. La Fig. 5.2(b) muestra una clasificación representativa (resultado de una realización Montecarlo) y la Tabla 5.2 muestra su correspondiente evaluación de desempeño. La Tabla 5.3 muestra los índices de desempeño promedio del análisis Montecarlo completo para ambos algoritmos. Se observa que ambos logran el grado "casi perfecto" de concordancia con el terreno simulado, de acuerdo a la escala de nivel de concordancia propuesta en Landis y Koch (1977) para los valores de kappa.

\subsection{Desempeño con datos reales}

En esta sección se aplica el algoritmo a datos reales MLC. Adicionalmente, también se aplica el algoritmo descripto en Horta et al. (2008b) con el número de clases 
Tabla 5.2: Desempeño de la clasificación para una realización

\begin{tabular}{|c|c|c|c|c|c|c|c|}
\hline & \multicolumn{4}{|c|}{ Clasificación } & exactitud & kарра \\
\hline & & azul & cyan & amarillo & rojo & \multirow{5}{*}{0.9897} & \multirow{5}{*}{0.9862} \\
\hline \multirow{4}{*}{ 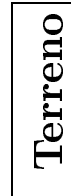 } & azul & 9591 & 0 & 14 & 395 & & \\
\hline & cyan & 0 & 10000 & 0 & 0 & & \\
\hline & amarillo & 0 & 0 & 10000 & 0 & & \\
\hline & rojo & 5 & 0 & 0 & 9995 & & \\
\hline
\end{tabular}

Tabla 5.3: Desempeño promedio para datos simulados

\begin{tabular}{|c|c|cc|}
\cline { 3 - 4 } \multicolumn{2}{c|}{} & \multicolumn{2}{c|}{ Indices promedio } \\
\cline { 3 - 4 } \multicolumn{2}{c|}{} & exactitud & kappa \\
\hline \multirow{⿱}{*}{} & Propuesto & $\mathbf{0 . 9 9 6 7}$ & $\mathbf{0 . 9 9 5 8}$ \\
& Horta & 0.9873 & 0.9830 \\
\hline
\end{tabular}

identificado por el algoritmo propuesto para comparar resultados de manera justa. Se utilizó una imagen AIRSAR $450 \times 500$ del área de San Francisco ${ }^{1}$. La Fig. 5.3 muestra la escena en falso color RGB. Se han definido cuatro zonas de control identificando las diferentes clases en la imagen: mar, vegetación, edificación y costa. Las primeras tres son visualmente distinguibles, mientras que la clase "costa" está oculta, pero ha sido identificada en trabajos previos (Lee y Pottier, 2009). Estas zonas son utilizadas como terreno verdadero para evaluar el desempeño usando la matriz de confusión.

Con el propósito de evaluar la consistencia y la exactitud, se aplicó el algoritmo propuesto 40 veces al conjunto de datos reales, con $\mathrm{PFA}=0,05$ y $n=3,4^{2}$. Se calcularon las matrices de confusión y los índices relacionados para cada clasificación resultante y el valor de kappa promedio se utilizó para evaluar el desempeño. El mismo procedimiento se aplicó con el algoritmo de Horta para comparar resultados.

\footnotetext{
${ }^{1}$ Disponible en https://earth.esa.int/web/polsarpro/airborne-data-sources

${ }^{2}$ Este valor fue previamente estimado en Horta et al. (2008a) utilizando del mismo conjunto de datos
} 


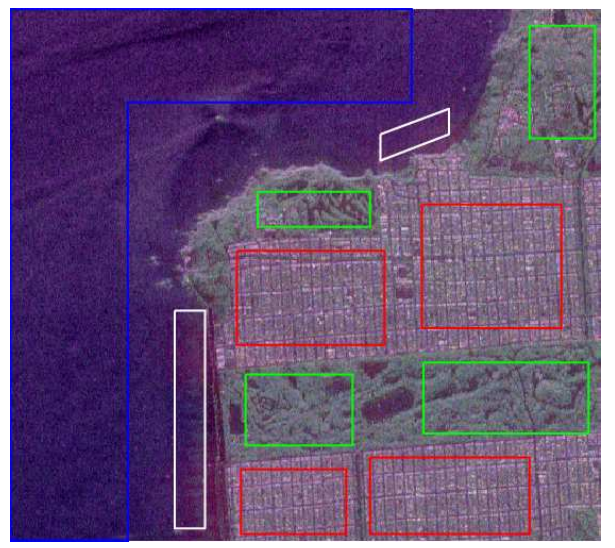

Figura 5.3: Imagen San Francisco. Rojo:|HH-VV|. Verde:|HV+VH|. Azul:|HH+VV|. Zonas de control: mar, edificado, vegetación, costa.

\subsubsection{Resultados}

El algoritmo propuesto identificó exitosamente las 4 clases presentes en todas las 40 ejecuciones, mientras que el algoritmo competidor logró hacerlo en 2 casos, siendo la clase "costa" pobremente identificada en la mayoría de ellos. La Fig. 5.4(a) muestra una clasificación representativa del algoritmo propuesto y la Tabla 5.4 indica la correspondiente matriz de confusión e índices relacionados. La Fig. 5.4(b) muestra la mejor clasificación obtenida con el algoritmo de Horta. Los índices promedio calculados sobre los casos exitosos figuran en la Tabla 5.5, mostrando que ambos algoritmos alcanzan el grado de acuerdo "sustancial" con las zonas de prueba definidas, de acuerdo a Landis y Koch (1977). Sin embargo, el algoritmo propuesto probó ser más confiable que el de Horta debido a su mayor tasa de clasificaciones correctas en la prueba.

Tabla 5.4: Desempeño del algoritmo propuesto para datos reales.

\begin{tabular}{|c|c|c|c|c|c|c|c|}
\hline & \multicolumn{4}{|c|}{ Clasificación } & exactitud & kappa \\
\hline & & mar & vegetación. & edificación & costa & \multirow{5}{*}{0.8850} & \multirow{5}{*}{0.8234} \\
\hline$\stackrel{0}{1}$ & mar & 63376 & 0 & 0 & 35 & & \\
\hline 0 & vegetación & 0 & 20733 & 362 & 780 & & \\
\hline \multirow{2}{*}{$\stackrel{\dot{0}}{\Theta}$} & edificación & 10 & 11291 & 23102 & 1657 & & \\
\hline & costa & 480 & 9 & 9 & 5378 & & \\
\hline
\end{tabular}




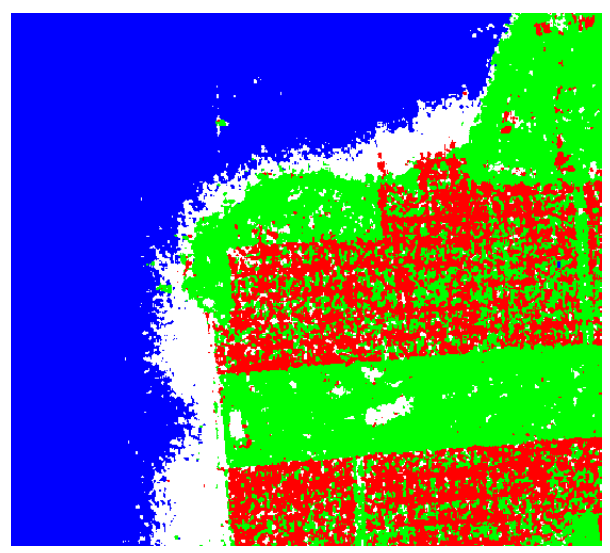

(a)

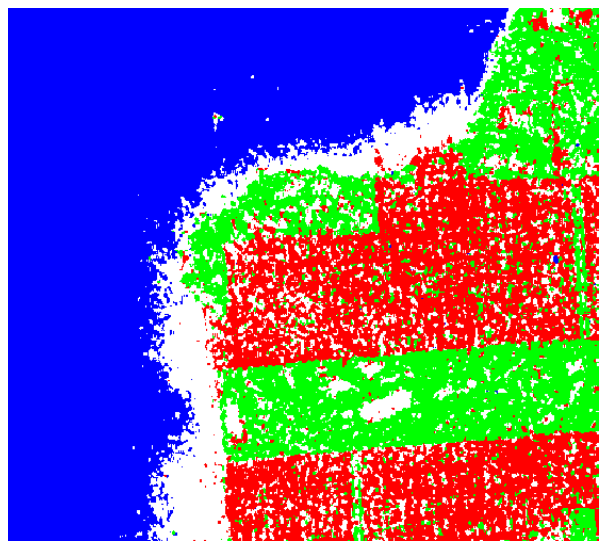

(b)

Figura 5.4: Ejemplos de resultados para datos reales. (a):Algoritmo propuesto. (b): Algoritmo de Horta.

Tabla 5.5: Desempeño promedio para datos reales.

\begin{tabular}{|c|c|c|c|}
\hline & \multicolumn{2}{|c|}{ İndices promedio } \\
\hline & & $\overline{\text { exactitud }}$ & kappa \\
\hline$\dot{00}$ & Prop & 0.8639 & 0.7933 \\
\hline$\psi_{1}$ & Horta & 0.8292 & 0.7382 \\
\hline
\end{tabular}

\subsection{Conclusiones}

Se desarrolló un algoritmo no supervisado para la clasificación de datos SAR polarimétricos basados en el modelo $\mathcal{G}_{p}^{0}$. El enfoque descendente de la etapa divisiónfusión provee el número de clases y una inicialización adecuada para el algoritmo EM. Debido a que el proceso inicia con una clase única para toda la imagen, la clasificación no depende de la inicialización. Adicionalmente, el algoritmo propuesto provee los estimados de máxima verosimilitud de la mezcla, a diferencia de los estimados en base a los momentos de los enfoques en Horta et al. (2008b) y Horta et al. (2008a), con el costo de un mayor costo computacional en el paso $\mathrm{M}$.

El algoritmo fue aplicado a datos simulados y reales. En el primer caso el análisis Montecarlo mostró baja dispersión en el número de clases identificado, lo que valida la etapa de división-fusión, y muy buenos resultados en términos de los índices exactitud y kappa promedio, lo que valida las etapas de estimación y clasificación. 
En el caso de datos reales, el algoritmo mostró consistencia al identificar las zonas de referencia en todos los casos con gran exactitud en la clasificación resultante.

También se han comparado los resultados obtenidos con los de Horta et al. (2008a) con inicialización con percentiles, el cual se basa en el mismo modelo $\mathcal{G}_{p}^{0} \mathrm{y}$ el algoritmo EM. A pesar de que los resultados parecen favorecer al algoritmo propuesto, deben hacerse más pruebas en diferentes conjuntos de datos para establecer conclusiones al respecto. La comparación presentada fue hecha sólo para comprobar la significancia de los resultados.

El trabajo futuro en esta línea se centrará en mejorar el proceso de divisiónfusión. El clasificador Wishart utilizado para estimar las matrices de covarianza podría ser reemplazado por otros métodos menos costosos computacionalmente. Además, a pesar de que no representa un inconveniente en este trabajo, la convergencia de la Ec. (5.8) debe ser estudiada. Una alternativa viable es adaptar el problema a las hipótesis del trabajo presentado en Formont et al. (2013) donde la convergencia ya ha sido probada. 


\section{A. Cálculo de EM para mezcla de densidades $\mathcal{G}_{p}^{0}$}

En este apartado se calculan las expresiones de los pasos E y M de la etapa de estimación del algoritmo de clasificación.

Dentro del contexto del algoritmo EM, cada dato MLC observado $Z \in \mathbb{C}^{3 \times 3}$ tiene asociado una variable aleatoria $v \in \mathbb{R}$ no observada que indica a cuál de las clases pertenece $Z$. La variable compuesta $U=[Z, v]$ caracteriza completamente al dato, de manera que si se conociera no sería necesario el proceso iterativo de EM, ya que estaría determinada la estructura de clases del conjunto. A $U$ se los denomina datos completos, a $Z$ datos observados y a $v$ datos ocultos. El algortimo EM trabaja sobre los datos observados para inferir la información de $v$.

\section{A.1. Cálculo de $Q\left(\boldsymbol{\theta} / \boldsymbol{\theta}^{\prime}\right)$}

De forma análoga a lo presentado en la Sec. 4.3.2, para estimar los parámetros de interés se define se define la función $Q\left(\boldsymbol{\theta} / \boldsymbol{\theta}^{\prime}\right)=\sum_{i=1}^{N} \mathcal{E}_{v_{i} \mid Z_{i} ; \boldsymbol{\theta}^{\prime}}\left[\log \left(f_{Z_{i}, v_{i} ; \boldsymbol{\theta}^{\prime}}\left(Z_{i}, v_{i} ; \boldsymbol{\theta}\right)\right)\right]$ y se maximiza en cada iteración respecto de las variables de interés.

Desarrollando la expresión de la esperanza condicional se tiene

$$
Q\left(\boldsymbol{\theta} / \boldsymbol{\theta}^{\prime}\right)=\sum_{i=1}^{N} \int_{-\infty}^{\infty} \log \left(f_{Z_{i}, v_{i} ; \boldsymbol{\theta}}\left(Z_{i}, v_{i} ; \boldsymbol{\theta}\right)\right) f_{v_{i} \mid Z_{i} ; \boldsymbol{\theta}^{\prime}}\left(v_{i} \mid Z_{i} ; \boldsymbol{\theta}^{\prime}\right) d v_{i} .
$$

Teniendo en cuenta que $v_{i}$ es una variable aleatoria discreta, la integral correspondiente a la esperanza condicional se expresa como una sumatoria sobre todos los valores posibles que puede tomar $v_{i}$, es decir, una sumatoria sobre las $K$ clases:

$$
Q\left(\boldsymbol{\theta} / \boldsymbol{\theta}^{\prime}\right)=\sum_{i=1}^{N} \sum_{j=1}^{K} \log \left(f_{Z_{i}, v_{i} ; \boldsymbol{\theta}}\left(Z_{i}, v_{i} ; \boldsymbol{\theta}\right)\right) P\left\{v_{i}=v_{j} \mid Z_{i} ; \boldsymbol{\theta}^{\prime}\right\} .
$$

Por otro lado, el Teorema de la Probabilidad Total permite reescribir la densidad condicional de la siguiente forma

$$
P\left\{v_{i}=v_{j} \mid Z_{i} ; \boldsymbol{\theta}^{\prime}\right\}=\frac{f_{Z_{i} \mid v_{i} \boldsymbol{\theta}^{\prime}}\left(Z_{i} \mid v_{i} ; \boldsymbol{\theta}^{\prime}\right) P\left\{v_{i}=v_{j} ; \boldsymbol{\theta}^{\prime}\right\}}{\sum_{l=0}^{K} f_{Z_{i} \mid v_{i} ; \boldsymbol{\theta}^{\prime}}\left(Z_{i} \mid v_{i} ; \boldsymbol{\theta}^{\prime}\right) P\left\{v_{i}=v_{l} ; \boldsymbol{\theta}^{\prime}\right\}} .
$$


El factor $P\left\{v_{i}=v_{j} ; \boldsymbol{\theta}^{\prime}\right\}$ en la última expresión corresponde a la probabilidad $a$ priori de la $j$-ésima clase, es decir, es el peso de dicha clase $w_{j}$ en la mezcla. Además, la densidad condicional $f_{Z_{i} \mid v_{i} ; \boldsymbol{\theta}^{\prime}}(\cdot)$ es la densidad asignada a los datos de cada clase, en este caso, la densidad $\mathcal{G}_{p}^{0}$. Por lo tanto, puede reescribirse la expresión anterior en términos de los pesos:

$$
P\left\{v_{i}=v_{j} \mid Z_{i} ; \boldsymbol{\theta}^{\prime}\right\}=\frac{f_{Z_{i} \mid v_{j} ; \boldsymbol{\theta}^{\prime}}\left(Z_{i} \mid v_{j} ; \boldsymbol{\theta}^{\prime}\right) w_{j}}{\sum_{l=0}^{K} f_{Z_{i} \mid v_{l} ; \boldsymbol{\theta}^{\prime}}\left(Z_{i} \mid v_{l} ; \boldsymbol{\theta}^{\prime}\right) w_{l}} .
$$

La expresión (5.16) se denota $\gamma_{i j}^{\prime}$ y representa la probabilidad de que el $i$-ésimo dato pertenezca a la $j$-ésima clase. El cálculo de este parámetro se realiza en el paso E para cada iteración de EM, donde $\boldsymbol{\theta}^{\prime}$ es el conjunto de parámetros estimado en la iteración previa.

La Ec. (5.14) puede escribirse en función de $\gamma_{i j}$ :

$$
Q\left(\boldsymbol{\theta} / \boldsymbol{\theta}^{\prime}\right)=\sum_{i=1}^{N} \sum_{j=1}^{K} \gamma_{i j}^{\prime} \log \left(f_{Z_{i}, v_{j} ; \boldsymbol{\theta}}\left(Z_{i}, v_{j} ; \boldsymbol{\theta}\right)\right) .
$$

Operando sobre densidad la conjunta $f_{Z_{i}, v_{j} ; \boldsymbol{\theta}}(\cdot)=f_{Z_{i} \mid v_{j} ; \boldsymbol{\theta}}(\cdot) w_{j}$, se obtiene

$$
Q\left(\boldsymbol{\theta} / \boldsymbol{\theta}^{\prime}\right)=\sum_{i=1}^{N} \sum_{j=1}^{K} \gamma_{i j}^{\prime}\left[\log \left(f_{Z_{i} \mid v_{j} ; \boldsymbol{\theta}}\left(Z_{i} \mid v_{j} ; \boldsymbol{\theta}\right)\right)+\log w_{j}\right]
$$

Finalmente, reemplazando $\left.f_{Z_{i} \mid v_{j} ; \boldsymbol{\theta}}\left(Z_{i} \mid v_{j} ; \boldsymbol{\theta}\right)\right)$ por la expresión de la densidad $\mathcal{G}_{p}^{0}(5.1)$, se obtiene la expresión $Q\left(\boldsymbol{\theta} / \boldsymbol{\theta}^{\prime}\right)$ en función de los parámetros de interés:

$$
\begin{aligned}
Q\left(\boldsymbol{\theta} / \boldsymbol{\theta}^{\prime}\right)= & \sum_{i=1}^{N} \sum_{j=1}^{K} \gamma_{i j}^{\prime}\left[n d \log n+(n-d) \log \left|Z_{i}\right|+\log \left(\Gamma\left(d n-\alpha_{j}\right)\right)+\right. \\
& \left(\alpha_{j}-d n\right) \log \left(n \operatorname{tr}\left(C_{j}^{-1} Z_{i}\right)+\gamma_{j}\right)-\log (h(n, d))-n \log \left|C_{j}\right|- \\
& \left.\log \left(\Gamma\left(-\alpha_{j}\right)\right)-\alpha_{j} \log \gamma_{j}\right]+\log w_{j}
\end{aligned}
$$




\section{A.2. Maximización}

Para obtener los estimadores, debe maximizarse $Q\left(\boldsymbol{\theta} / \boldsymbol{\theta}^{\prime}\right)$ respecto de cada parámetro $\left(\alpha_{j}, \gamma_{j}, C_{j}\right)$.

Operando respecto de $C_{j}$ :

$$
\begin{aligned}
& \underset{C_{j}}{\operatorname{argmáx}}\left(Q\left(\boldsymbol{\theta} / \boldsymbol{\theta}^{\prime}\right)\right)= \\
& \underset{C_{j}}{\operatorname{argmáx}}\left[\sum_{i=1}^{N}\left[\gamma_{i j}^{\prime}\left(\alpha_{j}-d n\right) \log \left(n \operatorname{tr}\left(C_{j}^{-1} Z_{i}\right)+\gamma_{j}\right)\right]-N_{j} n \log \left|C_{j}\right|\right],
\end{aligned}
$$

donde $N_{j}=\sum_{i=1}^{N} \gamma_{i j}^{\prime}$.

Derivando el argumento entre corchetes de (5.20) respecto de $C_{j}$ e igualando a cero se obtiene:

$$
\sum_{i=1}^{N} \gamma_{i j}^{\prime}\left(\alpha_{j}-d n\right) \frac{-C_{j}^{-1} Z_{i} C_{j}^{-1}}{n \operatorname{tr}\left(C_{j}^{-1} Z_{i}\right)+\gamma_{j}}-N_{j} n C_{j}^{-1}=0
$$

Despejando el segundo término, y luego multiplicando por $C_{j}$ primero a derecha y luego a izquierda resulta:

$$
C_{j}=-\frac{\left(\alpha_{j}-d n\right)}{N_{j} n} \sum_{i=1}^{N} \gamma_{i j} \frac{Z_{i}}{n \operatorname{tr}\left(C_{j}^{-1} Z_{i}\right)+\gamma_{j}}
$$

que es la expresión recursiva (5.8) indicada en el paso M del algoritmo.

Finalmente, para obtener la expresión $\mathcal{L}\left(\alpha_{j}, \gamma_{j}, C_{j}\right)$ de la Ec. (5.9), basta con identificar en la expresión de $Q\left(\boldsymbol{\theta} / \boldsymbol{\theta}^{\prime}\right)$ los términos dependientes de $\alpha_{j}$ o de $\gamma_{j}$ :

$$
\begin{aligned}
\mathcal{L}\left(\alpha_{j}, \gamma_{j}, C_{j}\right)= & \left.N_{j} \log \left(\Gamma\left(d n-\alpha_{j}\right)\right)-N_{j} \log \left(\Gamma\left(-\alpha_{j}\right)\right)-N_{j} \alpha_{j} \log \gamma_{j}\right]+ \\
& \sum_{i=1}^{N} \gamma_{i j}^{\prime}\left(\alpha_{j}-d n\right) \log \left(n \operatorname{tr}\left(C_{j}^{-1} Z_{i}\right)+\gamma_{j}\right)
\end{aligned}
$$




\section{Capítulo 6}

\section{Conclusiones}

En esta tesis se realizaron distintos aportes en la rama del procesamiento de datos SAR relacionados con la clasificación de datos polarimétricos.

En primer lugar se estudió la geometría del problema SAR para entender el proceso de formación de imágenes. Se centró la atención en el SAR aerotransportado en modo stripmap con estrabismo nulo, aunque los principios son aplicables al SAR satelital, con las correspondientes correcciones por curvatura de la Tierra.

Partiendo de una geometría sencilla de desplazamiento rectilíneo y tierra plana, y del tipo de señales emitidas por el radar pulsado, se dedujo la señal recibida por la antena en respuesta a un objetivo puntual. Utilizando la respuesta del filtro adaptado al pulso emitido se determinó la resolución del sistema en la dirección de rango. Por otro lado, el concepto de procesamiento Doppler y apertura sintética permitieron identificar en la dirección de acimut la naturaleza chirp de la señal en tiempo lento. El procesamiento de la misma con el filtro adaptado permitió establecer la resolución SAR en acimut como una función dependiente únicamente del largo físico de la antena, que es la característica más sobresaliente del sistema SAR.

La identificación de la respuesta SAR como una señal bidimensional que se desarrolla en las direcciones de rango y acimut, junto con la simplificación parabólica de la fase, permitieron la implementación de filtros adaptados independientes en cada dirección, que es la base del algoritmo de enfoque RDA. Una implementación del mismo fue utilizada para enfocar datos sintéticos y visualizar cada etapa del pro- 
cesamiento. Posteriormente, una implementación más completa fue utilizada para enfocar datos reales de la misión SARAT, con buenos resultados.

Esta primera parte del trabajo, centrada fundamentalmente en el procesamiento digital, permitió la comprensión de la formación de la imagen SAR compleja (datos SLC) a partir de los datos crudos.

Posteriormente se trató la representación de los datos polarimétricos y el modelado estadístico utilizados en los algoritmos de clasificación propuestos. Partiendo de la ecuación de radar y del concepto de sección transversal para el radar convencional, se extendió el concepto al caso de objetivos extendidos y se definió la matriz de dispersión para representar los datos SLC polarimétricos básicos. En base al fenómeno de speckle, característico de los sistemas donde existe interacción coherente de las ondas, se estableció la diferencia entre los tipos de datos homogéneos y no homogéneos. En los primeros, donde la longitud de onda de la señal es mucho menor a la rugosidad del terreno, el mismo puede verse como no texturado. En estos casos, los datos SLC siguen la estadística gaussiana y la correspondiente matriz de covarianza muestral tiene distribución Wishart.

Por otro lado, cuando la textura del terreno no puede despreciarse, es necesario recurrir al modelo multiplicativo donde los datos son descriptos por el producto de dos variables aleatorias, una que describe al efecto speckle y otra al terreno.

En este contexto se presentó a la distribución $\mathcal{G}_{p}^{0}$ para modelar los datos MLC polarimétricos. Esta distribución resulta del producto de una distribución Gamma Inversa y una Wishart compleja, y combina las características de versatilidad para ajustarse a datos de diverso grado de heterogeneidad y tratabilidad matemática.

Tanto el modelo gaussiano como el modelo $\mathcal{G}_{p}^{0}$ fueron utilizados como base de los algoritmos de clasificación presentados en los Caps. 4 y 5 respectivamente, que es donde esta tesis realiza su principal aporte.

En el Cap. 4 se desarrolló un algoritmo de clasificación no supervisado para datos polarimétricos homogéneos. En base al modelo gaussiano, se propuso un modelo de mezcla de densidades gaussianas complejas para describir a los datos SLC polarimétricos, donde cada componente de la mezcla modela una clase presente en el conjunto de datos. El algoritmo no necesita de información previa para realizar la 
clasificación, y todos los parámetros son estimados mediante el método EM. Previo a la estimación, el algoritmo tiene una etapa de selección del modelo basada en la distancia KL que permite determinar el número de clases presentes en el conjunto de datos. El algoritmo fue probado en múltiples conjuntos de datos simulados y reales, obteniendo resultados satisfactorios en ambos casos.

En el Cap. 5 se propuso un algoritmo de clasificación no supervisado para datos polarimétricos no-homogéneos. Se utilizó un modelo de mezcla de densidades $\mathcal{G}_{p}^{0}$ para los datos MLC y el algoritmo EM para la estimación de sus parámetros. En este caso, la etapa de selección de modelo se realizó en base a un test de hipótesis que compara matrices de covarianza, lo que permitió diferenciar clases con suficiente distancia estadística entre ellas como para considerarlas clusters diferentes. Al igual que en caso anterior, el algoritmo se probó satisfactoriamente en datos simulados y reales, y mostró buen desempeño en comparación con algoritmos precedentes basados en el mismo modelo.

En ambos algoritmos se dedicó especial esfuerzo en determinar el orden del modelo, con el objeto de expresar los datos con la mínima complejidad y reducir el número de variables a estimar. Respecto al caso de datos homogéneos, partiendo de un número arbitrario de clases el mismo se reduce progresivamente hasta encontrar el número óptimo en el sentido BIC, evitando fusionar datos de clases bien diferenciadas en el sentido KL. Para el caso de datos no homogéneos, el intento de extender la misma estrategia para datos texturados resultaría inviable, debido a la falta de una expresión cerrada para la distancia KL del modelo $\mathcal{G}_{p}^{0}$. Se optó entonces por un enfoque descendente (top-down), partiendo de una clase única e incrementando su número a medida que el algoritmo halla evidencia estadística suficiente mediante el test de hipótesis.

Ambas estrategias representan un incremento en el tiempo de cómputo, sin embargo, permiten la clasificación sin contar con información previa del terreno, como datos de entrenamiento o segmentaciones iniciales.

Respecto del desempeño, en ambos algoritmos se utilizó la matriz de confusión y los parámetros asociados para compararlos con los algoritmos competidores. En el caso de datos simulados, esta forma de medir desempeño en la clasificación es 
natural, ya que el terreno verdadero es conocido y se define previamente. Sin embargo, para datos reales donde el terreno es desconocido, es necesario definirlo en base zonas de control donde se presume que existe una sola clase por zona. Si bien este procedimiento es el más comúnmente utilizado en la bibliografía, puede introducir errores si las clases no son fácilmente distinguibles.

Debido a esto, se propuso al índice de Davies-Bouldin como medida de desempeño. Este índice se calcula en base a los datos etiquetados, independientemente del modelo utilizado y de los datos originales. Sin embargo, mostró ser dependiente del número de clases, por lo que sólo es válido utilizarlo para comparar clasificaciones con igual numero de clases resultante. Su utilización como medida de desempeño entre dos clasificaciones cualquiera requiere una redefinición de los parámetros de dispersión y del centro de clases en los que se basa.

En conclusión, el aporte novedoso de este trabajo radica en el desarrollo de dos algoritmos de clasificación no supervisados para datos polarimétricos, con etapas de selección de orden de modelo que no requieren información previa del terreno. Para el caso de datos homogéneos, si bien el modelo de mezclas de fdp's gaussianas y el algoritmo EM son extensamente utilizados, se aplican generalmente sobre datos de intensidad o amplitud, y el número de clases es un parámetro requerido. En el algoritmo propuesto se conserva la información de los tres canales simultáneamente, a la vez que se mantiene la simplicidad de la estadística gaussiana. Respecto del algoritmo para datos con textura, la fdp $\mathcal{G}_{p}^{0}$ es una distribución del estado del arte, y su utilización en modelo de mezclas es limitada. En el algoritmo presentado, se utilizan con éxito junto al algoritmo EM y se calculan sus estimadores de máxima verosimilitud, lo que evita las restricciones propias del método de los momentos.

\subsection{Trabajo Futuro}

En el desarrollo del trabajo de tesis, se ha observado que los algoritmos de clasificación son computacionalmente costosos. Esto se debe a que los estimación de los parámetros del modelo debe ser resuelta utilizando métodos numéricos iterativos, como EM, aun cuando los modelos sean tan simples como la mezcla de distribuciones 
gaussianas. Por lo cual, al aumentar las dimensiones de la imagen, el tiempo de procesamiento crece de forma exponencial. Una posible forma de atacar este problema sería fraccionar la imagen en bloques y asignar cada uno a un procesador independiente funcionando en paralelo. Debido a que se trata de algoritmos de clasificación no supervisados, el desafío consiste en fusionar los resultados locales para generar una clasificación global.

Este problema es similar al que se presenta en redes de sensores con procesamiento distribuido, donde cada sensor no tiene acceso a los datos de sus vecinos. Para generar una estimación global, se requiere un centro de fusión que centraliza la información de todos los sensores de la red. También existen técnicas de procesamiento descentralizado que varían según el tipo de información que se intercambia entre los sensores. Recientemente se han desarrollado algoritmos que permiten alcanzar una estimación global consensuando las estimaciones locales, sin necesidad de un centro de fusión (Dimakis et al., 2010; Olfati-Saber et al., 2007; Aysal et al., 2009). Como trabajo a futuro, se buscará extender estas técnicas de estimación consensuada al problema de clasificación de imágenes SAR de grandes dimensiones.

Adicionalmente, y fuera del contexto SAR, se estudiará la posibilidad de aplicar las técnicas desarrolladas al problema de clasificación de datos de radar meteorológico, donde resulta de interés identificar el tipo de meteoro (lluvia, nieve, granizo, etc.) y su tamaño, a partir de los datos de dispersión en la atmósfera. Si bien los fenómenos observados son de naturaleza diferente a los del problema SAR, la respuesta de cada meteoro difiere para distintas polarizaciones, por lo que los datos son descriptos en base a la matriz de dispersión (Bringi y Chandrasekar, 2001, Cap. 5). Se espera poder utilizar un modelo de mezclas de densidades y método EM para su clasificación. 


\section{Bibliografía}

Anfinsen, S., A. Doulgeris, y T. Eltoft. Estimation of the equivalent number of looks in polarimetric synthetic aperture radar imagery. IEEE Trans. Geosci. Remote Sens., 47(11):3795-3809 (2009). ISSN 0196-2892.

Aysal, T. C., M. E. Yildiz, A. D. Sarwate, y A. Scaglione. Broadcast gossip algorithms for consensus. IEEE Transactions on Signal Processing, 57(7):2748-2761 (2009). ISSN 1053-587X.

Bringi, V. N. y V. Chandrasekar. Polarimetric Doppler Weather Radar. Cambridge University Press (2001). ISBN 9780511541094. Cambridge Books Online.

Celeux, G. y G. Govaert. A classification EM algorithm for clustering and two stochastic versions. Computational Statistics and Data Analysis, 14(3):315 - 332 (1992). ISSN 0167-9473.

Cheney, M. A mathematical tutorial on synthetic aperture radar. SIAM Rev., 43(2):301-312 (2001). ISSN 0036-1445.

Cloude, S. y E. Pottier. An entropy based classification scheme for land applications of polarimetric SAR. IEEE Trans. Geosci. Remote Sens., 35(1):68-78 (1997). ISSN 0196-2892.

Conradsen, K., A. A. Nielsen, J. Schou, y H. Skriver. A test statistic in the complex Wishart distribution and its application to change detection in polarimetric SAR data. IEEE Trans. Geosci. Remote Sens., 41(1):4-19 (2003).

Conte, E., A. De Maio, y G. Ricci. Recursive estimation of the covariance matrix of a compound-Gaussian process and its application to adaptive CFAR detection. IEEE Trans. Signal Process., 50(8):1908-1915 (2002). ISSN 1053-587X. 
Cumming, I. G. y F. H. Wong. Digital Processing of Synthetic Aperture Radar Data - Algorithms and Implementation. Artech House (2005). ISBN 978-1-58053-058-3.

Dabboor, M. y M. Shokr. A new likelihood ratio for supervised classification of fully polarimetric SAR data: An application for sea ice type mapping. ISPRS Journal of Photogrammetry and Remote Sensing, 84(0):1 - 11 (2013). ISSN 0924-2716.

Davies, D. L. y D. W. Bouldin. A cluster separation measure. IEEE Trans. Pattern Anal. Mach. Intell., PAMI-1(2):224-227 (1979). ISSN 0162-8828.

Dempster, A. P., N. M. Laird, y D. B. Rubin. Maximum likelihood from incomplete data via the EM algorithm. Journal of the Royal Statistical Society. Series B (Methodological), 39(1):pp. 1-38 (1977). ISSN 00359246.

Dimakis, A. G., S. Kar, J. M. F. Moura, M. G. Rabbat, y A. Scaglione. Gossip algorithms for distributed signal processing. Proceedings of the IEEE, 98(11):18471864 (2010). ISSN 0018-9219.

Doulgeris, A. P., V. Akbari, y T. Eltoft. Automatic polsar segmentation with the U-distribution and Markov random fields. En Synthetic Aperture Radar, 2012. EUSAR. 9th European Conference on, págs. 183-186 (2012).

Dutta, A. y K. Sarma. SAR image segmentation using wavelets and Gaussian mixture model. En Signal Processing and Integrated Networks (SPIN), 2014 International Conference on, págs. 466-770 (2014).

European Space Agency. ESA sample SAR datasets (2014). (Accessed 11 December, 2014).

Fernandez Michelli, J., M. Hurtado, J. Areta, y C. Muravchik. Polarimetric SAR image segmentation using CEM algorithm. Latin America Transactions, IEEE (Revista IEEE America Latina), 12(5):910-914 (2014). ISSN 1548-0992.

Fernández Michelli, J., M. Hurtado, J. Areta, y C. Muravchik. Clasificación de imágenes SAR polarimétricas utilizando el método EM y el modelo $\mathcal{G}_{p}^{0}$. En de Trabajo en Procesamiento de la Información y Control, X. R., ed., XVI Reunión de Trabajo en Procesamiento de la Información y Control (2015).

Fjortoft, R., A. Lopes, J. Bruniquel, y P. Marthon. Optimal edge detection and edge localization in complex SAR images with correlated speckle. IEEE Trans. Geosci. Remote Sens., 37(5):2272-2281 (1999). ISSN 0196-2892. 
Formont, P., J.-P. Ovarlez, y F. Pascal. On the use of matrix information geometry for polarimetric SAR image classification. En Nielsen, F. y R. Bhatia, eds., Matrix Information Geometry, págs. 257-276. Springer Berlin Heidelberg (2013). ISBN 978-3-642-30231-2.

Freitas, C. C., A. C. Frery, y A. H. Correia. The polarimetric G distribution for SAR data analysis. Environmetrics, 16(1):13-31 (2005).

Frery, A., H.-J. Muller, C. Yanasse, y S. Sant'Anna. A model for extremely heterogeneous clutter. IEEE Trans. Geosci. Remote Sens., 35(3):648-659 (1997). ISSN 0196-2892.

Frery, A. C., J. Jacobo-Berlles, J. Gambini, y M. Mejail. Polarimetric SAR image segmentation with B-Splines and a new statistical model. CoRR, abs/1207.3944 (2012).

Hochwald, B. y A. Nehorai. Polarimetric modeling and parameter estimation with applications to remote sensing. IEEE Trans. Signal Process., 43(8):1923-1935 (1995). ISSN 1053-587X.

Horta, M. M., N. Mascarenhas, y A. C. Frery. A comparison of clustering fully polarimetric SAR images using SEM algorithm and $\mathcal{G}_{p}^{0}$ mixture modelwith different initializations. En Proc. 19th International Conf. on Pattern Recognition (ICPR 2008), págs. 1-4 (2008a). ISSN 1051-4651.

Horta, M. M., N. Mascarenhas, A. C. Frery, y A. Levada. Clustering of fully polarimetric SAR data using finite $\mathcal{G}_{p}^{0}$ mixture model and SEM algorithm. En Proc. 15th International Conf. on Systems, Signals and Image Processing (IWSSIP 2008), págs. 81-84 (2008b).

Kayabol, K. y J. Zerubia. Unsupervised amplitude and texture classification of SAR images with multinomial latent model. IEEE Trans. Image Process., 22(2):561572 (2013). ISSN 1057-7149.

Kong, J., A. Swartz, H. Yueh, L. Novak, y R. Shin. Identification of terrain cover using the optimum polarimetric classifier. Journal of Electromagnetic Waves and Applications, 2(2):171-194 (1988).

Konishi, S. y G. Kitawa. Information Criteria and Statistical Modeling. Springer Series in Statistics. Springer (2008). 
Landis, J. R. G. y G. Koch. The measurement of observer agreement for categorical data. Biometrics, 33(1):159-174 (1977). ISSN 0006341X, 15410420.

Lee, J., D. Schuler, R. Lang, y K. Ranson. K-distribution for multi-look processed polarimetric SAR imagery. En Geoscience and Remote Sensing Symposium, 1994. IGARSS '94. Surface and Atmospheric Remote Sensing: Technologies, Data Analysis and Interpretation., International, tomo 4, págs. 2179-2181 vol.4 (1994).

Lee, J. S., M. Grunes, T. Ainsworth, L.-J. Du, D. Schuler, y S.R.Cloude. Unsupervised classification using polarimetric decomposition and the complex Wishart classifier. IEEE Trans. Geosci. Remote Sens., 37(5):2249-2258 (1999). ISSN 0196-2892.

Lee, J. S. y E. Pottier. Polarimetric Radar Imaging: from Basics to Applications. Optical science and Engineering. CRC Press, Taylor and Francis Group (2009). ISBN 978-1-4200-5497-2.

McLachlan, G. J. y T. Krishnan. The EM Algorithm and Extensions. Wiley Series in Probability and Statistics. John Wiley and Sons, 2 edición (2008). ISBN 9780-471-20170-0.

Noiboar, A. y I. Cohen. Anomaly detection based on wavelet domain GARCH random field modeling. IEEE Trans. Geosci. Remote Sens., 45(5):1361-1373 (2007). ISSN 0196-2892.

Olfati-Saber, R., J. A. Fax, y R. M. Murray. Consensus and cooperation in networked multi-agent systems. Proceedings of the IEEE, 95(1):215-233 (2007). ISSN 00189219.

Pascal, F., Y. Chitour, J. Ovarlez, P. Forster, y P. Larzabal. Covariance structure maximum-likelihood estimates in compound Gaussian noise: Existence and algorithm analysis. IEEE Trans. Signal Process., 56(1):34-48 (2008). ISSN 1053-587X.

Pascual, J., J. Fernández Michelli, N. von Ellenrieder, M. Hurtado, J. Areta, y C. Muravchik. Image classification by means of CEM algorithm based on a GARCH-2D data model. Latin America Transactions, IEEE (Revista IEEE America Latina), 12(5):877-882 (2014). ISSN 1548-0992.

Richards, M. Fundamentals of Radar Signal Processing. McGraw-Hill (2005). ISBN 0-07-144474-2. 
Runnalls, A. Kullback-Leibler approach to Gaussian mixture reduction. IEEE Trans. Aerosp. Electron. Syst., 43(3):989-999 (2007). ISSN 0018-9251.

Sánchez-Lladó, F. J., G. Pajares, y C. López-Martínez. Improving the Wishart synthetic aperture radar image classifications through deterministic simulated annealing. ISPRS Journal of Photogrammetry and Remote Sensing, 66(6):845 - 857 (2011). ISSN 0924-2716.

Schlutz, M. Synthetic Aperture Radar Imaging Simulated in MATLAB. Proyecto Fin de Carrera, California Polytechnic State University, San Luis Obispo (2009). Schwarz, G. Estimating the dimension of a model. The Annals of Statistics, 6(2):461-464 (1978).

Uhlmann, S. y S. Kiranyaz. Classification of dual- and single polarized SAR images by incorporating visual features. ISPRS Journal of Photogrammetry and Remote Sensing, 90(0):10 - 22 (2014). ISSN 0924-2716.

van Zyl, J. Unsupervised classification of scattering behavior using radar polarimetry data. IEEE Trans. Geosci. Remote Sens., 27(1):36-45 (1989). ISSN 0196-2892.

Wang, B.-C. Digital Signal Processing Techniques and Applications in Radar Image Processing. Wiley-Interscience, New York, NY, USA (2008). ISBN 0470180927, 9780470180921.

Yuan, L., J. Song, W. Xue, y W. Zhao. SAR image classification based on MAP via the EM algorithm. En Intelligent Control and Automation, 2006. WCICA 2006. The Sixth World Congress on, tomo 2, págs. 10116-10120 (2006).

Yueh, H. A., A. A. Swartz, J. A. Kong, R. T. Shin, y L. Novak. Optimal classification of terrain cover using normalized polarimetric data. Journal of Geophysical Research (1993). 
